LBL-34431

UC-350

\title{
Analysis of PG\&E's Residential End-Use Metered Data to Improve Electricity Demand Forecasts - Final Report
}

\author{
Joseph H. Eto and Mithra M. Moezzi \\ Energy \& Environment Division \\ Lawrence Berkeley Laboratory \\ University of California \\ Berkeley, CA 94720
}

December 1993

The work described in this report was funded by the California Institute for Energy Efficiency (CIFE) for the Pacific Gas and Electric Company (PG\&E) and for the Califormia Energy Commission (CEC) and by the Assistant Secretary for Energy Efficiency and Renewable Energy, Office of Utility Technologies, Office of Energy Management Division of the U.S. Department of Energy under Contract No. DE-AC03-76SF00098.

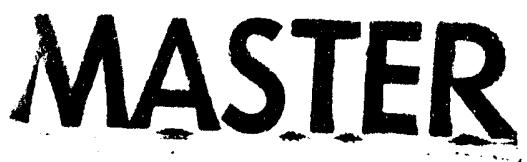


Executive Summary $\ldots \ldots \ldots \ldots \ldots \ldots \ldots \ldots \ldots \ldots \ldots$

Chapte: 1

Introduction

Chapter 2

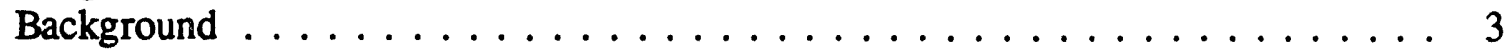

The PG\&E Appliance Metering Project . . . . . . . . . . . . 3

Electricity Load Shape Forecasting Models . . . . . . . . . . . . . . . . 4

PG\&E and CEC Peak Demand Forecasting Model Specifications . . . . . . 5

Chapter 3

Daily Energy Model $\ldots \ldots \ldots \ldots \ldots$

Load Data . . . . . . . . . . . . . . . . . . . . . . . . 11

Weather Station Assignment and Explanatory Variable Definition . . . . 12

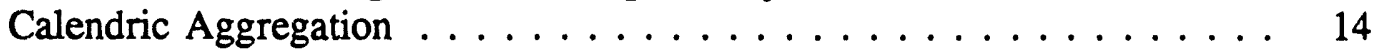

Linear Regression Models . . . . . . . . . . . . . . . . . . . . . . . . . . . . . . . . . . . . .

Assessing Model Fit . . . . . . . . . . . . . . . . . 16

Chapter 4

Binned Hourly Energy Load Shapes . . . . . . . . . . . . . . . . . . . 23

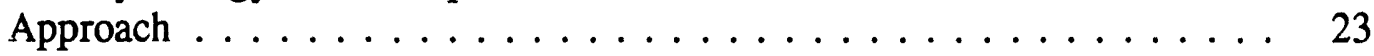

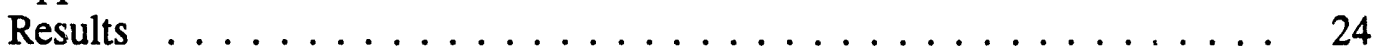

Chapter 5

Hourly Energy Models . . . . . . . . . . . . . . . . . . . . . . . . 27

HELM Hourly iNeather Response Functions . . . . . . . . . . . . . 27

Weather and Lodd Data . . . . . . . . . . . . . . . . . . . 28

Exploratory Regression Results for CEC Region 3 . . . . . . . . . . . . . . . 29

Regression Results for the Remaining CEC Regions . . . . . . . . . . . . . 31

Additional Thoughts on Hourly Model Development . . . . . . . . . . . 32 
Chapter 6

Comparison of Cooling End Use Models . . . . . . . . . . . . . . 45

Computation of Predicted Loads . . . . . . . . . . . . . . . 46

Methods of Comparison . . . . . . . . . . . . . . . . 50

Results of the Model Comparisons for CEC Regions . . . . . . . . . 51

Results of Model Comparisons for PG\&E Zones . . . . . . . . . . . . . 54

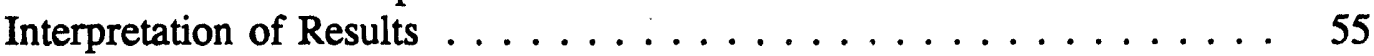

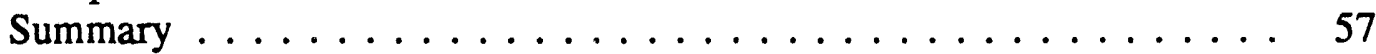

Chapter 7

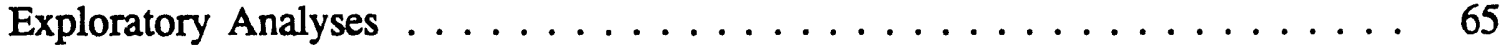

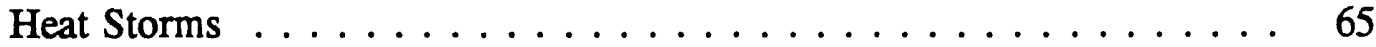

Heat Pump Compressor . . . . . . . . . . . . . . 72

Room Air Conditioner . . . . . . . . . . . . . . . . . . . 76

Alternate Weather for Modeling CEC Region $4 \ldots \ldots$. . . . . . . . . . . . 82

Alternative Definitions of Hourly Load Shape Bins . . . . . . . . . . . 84

Separate Modeling of Peak Days . . . . . . . . . . . . . . . 91

Chapter 8

Directions for Future Research . . . . . . . . . . . . . . . . . . 93

Respecification of Binned Load Shapes . . . . . . . . . . . . . . . . . 93

Peak Demand versus Load Shape Forecasting . . . . . . . . . . . . . . . . 94

Sample Weight Development . . . . . . . . . . . . . . . . . . . . 94

Weather Data for Forecasting ................. 95

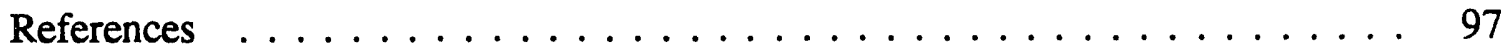

Appendix A

Data Handling Conventions . . . . . . . . . . . . . . . . . . 99

Geographic Coding . . . . . . . . . . . . . . . . . . . . . . . . . . . . . . . 99

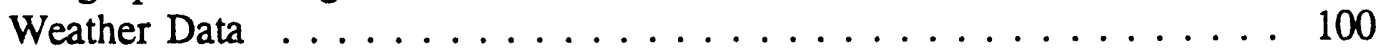

PG\&E End-Use Load Data . . . . . . . . . . . . . . . . . . . . 101

Time Conventions ....................... 103

Appendix B

Plots of Hourly Binned

Load Shapes for Chapter $4 \ldots \ldots \ldots$. . . . . . . . . . . . . 105

Appendix C

Plots of Additional Model

Comparisons from Chapter $5 \ldots \ldots \ldots \ldots \ldots \ldots$ 


\section{Appendix D}

HELM Model Implementation

of Project Results

131

Background

132

Approach and Findings $\ldots \ldots \ldots \ldots \ldots \ldots \ldots \ldots \ldots \ldots \ldots \ldots$

Discussion ...................... 133

Recommendation .................... 134 


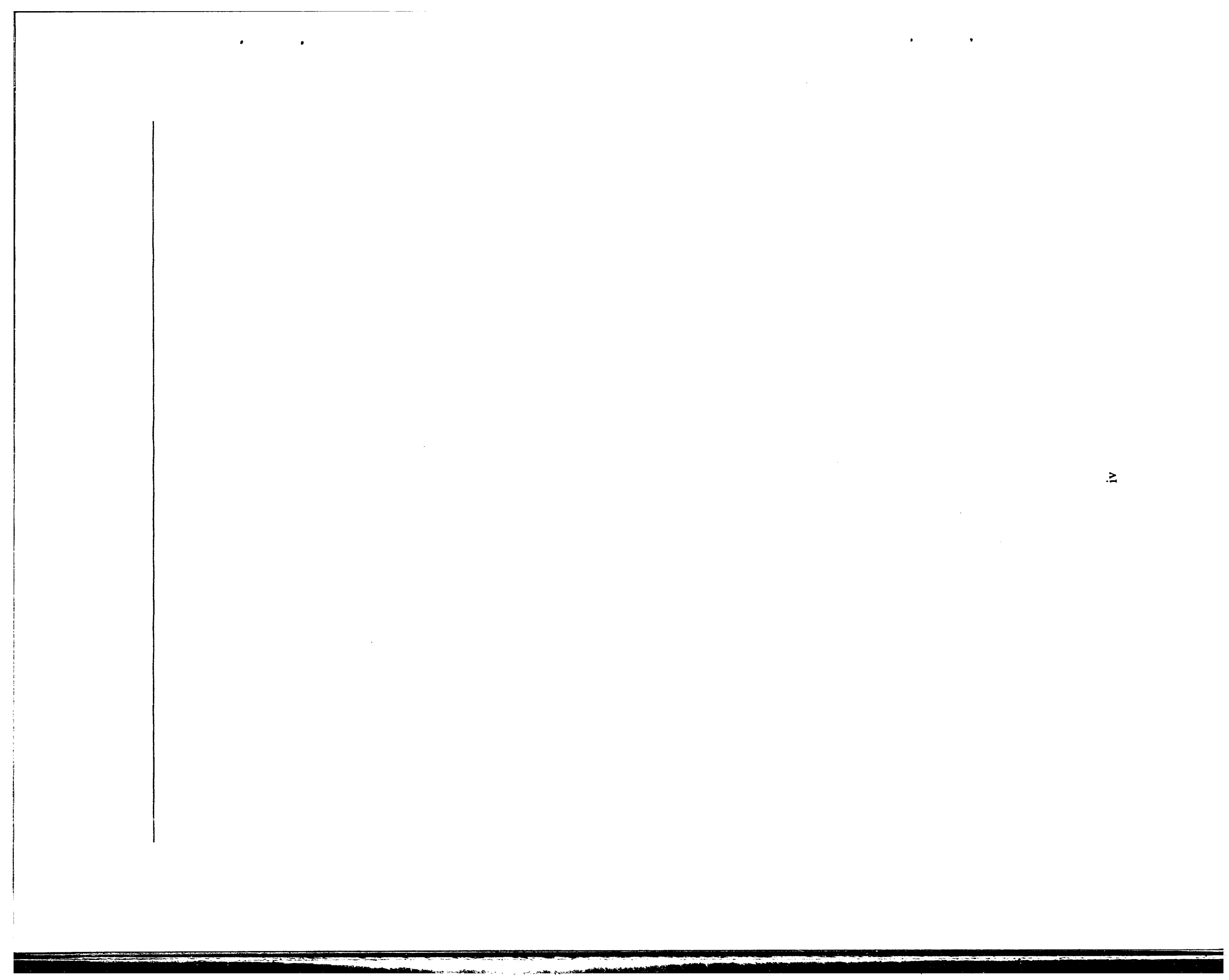


Table 2-1. Annual UEC Load Factor (LF), and Coincidence Factor (CF) for Central Air Conditioning, PG\&E Zones . . . . . . . . . . 7

Table 2-2. Annual UEC, Load Factor (LF), and Coincidence Factor (CF) for Central Air Conditioning, CEC Regions . . . . . . . . . . . 8

Table 3-1. Weather Stations Assigned to PG\&E Zones and CEC Regions for Cooling End-Use Models Based on 1985-1989 AMP Data . . . . 18

Table 3-2. Variables Used in Daily Regressions . . . . . . . . . . . . . . . 19

Table 3-3. Summary of Regression Results for CEC Regions 2,3, and 4 . . 20

Table 3-4. Summary of Regression Results for PG\&E Zones S,R, and X . . 21

Table 4-1. Cooling Load Bins by PG\&E Zone for Spring, Summer and Fall

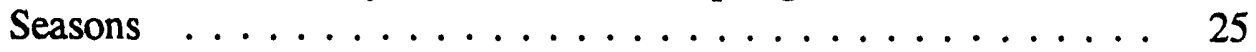

Table 4-2. Cooling Load Bins by CEC Region for Spring, Summer, and Fall

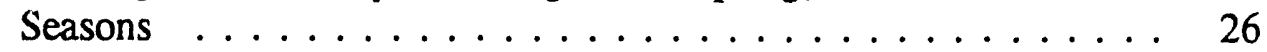

Table 5-1. Variables Used in Hourly Central Air Conditioner Load Regressions ... . . . . . . . . . . . . . . . . . 34

Table 5-2. Summary of Model 1 Hourly Regressions for CEC Region 3 Summer Season . . . . . . . . . . . . . . . . . . . 35

Table 5-3. Summary of Model 2 Hourly Regressions for CEC Region 3 Summer Season . . . . . . . . . . . . . . . . . . . 36

Table 5-4. Summary of Model 3 Hourly Regressions for CEC Region 3 Summer Season . . . . . . . . . . . . . . . . . . . 37

Table 5-5. Summary of Model 3 Hourly Regressions for CEC Region 2

Table 5-6. Summary of Model 3 Hourly Regressions for CEC Region 4 Summer Season . . . . . . . . . . . . . . . . . . . . . . 39

Table 5-7. Summary of Hours-pooled Regression Results for Summer CEC Region $3 \ldots \ldots \ldots$. . . . . . . . . . . . . . . 40

Table 5-8. Comparison of Prediction Errors in Selected Hours for Model 3 Based on Peak Day Subset to Model 3 Based on All Summer Days, for CEC Region $3 \ldots \ldots$. . . . . . . . . . . 41

Table 6-1. Models Compared for CEC regional Load Shape Forecasting . . . 58

Table 6-2. Models Compared for PG\&E Zonal Load Shape Forecasting . . . 59

Table 6-3. Comparison of Model Performance on Top Two System Load Days 1985-1989, by CEC Region . . . . . . . . . . . . . . . . 60

Table 7-1. Annual Number of Heat Storms for PG\&E Weather Stations, as Defined by Daily Average Dry Bulb Temperature . . . . . . . . 
Table 7-2. Annual Number of Heat Storms for PG\&E Weather Stations, as Defined by Day's Maximum Hourly Dry Bulb Temperature . . . . 70

Table 7-3. Number of Heat Pump Compressors Metered by CEC Region and PG\&E Zones . . . . . . . . . . . . . . . . . . 74

Table 7-4. Average Monthly UECs for Heat Pump Compressor (HPC) and Central Air Conditioner (CAC) for CEC Region 3, PG\&E Zone R, and PG\&E Zone S . . . . . . . . . . . . . 74

Table 7-5. Annual UEC for Room Air Conditioning, CEC Regions . . . . . 79

Table 7-6. Annual UEC for Room Air Conditioning, PG\&E Zones . . . 80

Table 7-7. Summary of Regression Results for Daily Room Air Conditioner Load . . . . . . . . . . . . . . . . . . . . 81

Table 7-8. Comparison of CEC region 4 Daily Central Air Conditioner Load Regression Model Fits for Three Alternative Weather Stations ..................... 83

Table 7-9. Distribution of Hour of Day in Which Maximum Load Occurred for Region 3 Summer AMP Sample Average Load Shapes . . . . . . . . . . . . . . . . . . . . . . 87

Table 7-10. Comparison of Prediction Errors in Selected Hours for Model 3 Based on Peak Day Subset to Model 3 Based on All Summer Days, for CEC Region $3 \ldots \ldots$. . . . . . . . . . . . . 92

Table A-1. Number of Central and Room Air Conditioners Metered by CEC Region and PG\&E Zone . . . . . . . . . . . . . . . . 103

Table A-2. Comparison of Geographic Assignments Between PG\&E Zones and CEC Regions . . . . . . . . . . . . . . . . . . . 104 


\section{Figures}

Figure 2-1. PG\&E Climate Zones . . . . . . . . . . . . . . 9

Figure 2-2. CEC Climate Regions $1-5 \ldots \ldots \ldots \ldots$

Figure 3-1. Time Series Plot of Residuals from Daily WRFs for

CEC Regions 2,3, and 4, 1985-1989 (winter omitted) . . . . . . 22

Figure 5-1. Regression Coefficients for Model 3 Summer Weekday . . . . . . . 42

Figure 5-2. Temporal Patterns in Residuals for 7 p.m. Hourly Load Model . . . 43

Figure $6-1$. Measures of Hourly Fit . . . . . . . . . . . . . . 61

Figure $6-2 . \quad$ Components of a Boxplot . . . . . . . . . . . . . . . 62

Figure 6-3. Distribution of Model Residuals (obs.-pred.) for Peak Day

Subset by CEC Region . . . . . . . . . . . . . . . 63

Figure 6-4. Distribution of Model Residuals (obs.-pred.) for Peak Day

Subset by PG\&E Zone . . . . . . . . . . . . . . . . . . 64

Figure 7-1. Distribution of Residuals from Daily WRF by Length of

Heat Storm, PG\&E Zone R . . . . . . . . . . . 71

Figure 7-2. Comparison of Daily Heat Pump Compressor and Central Air

Conditioner Loads . . . . . . . . . . . . . . . . . . 75

Figure 7-3. Distribution of Hourly Central Air Conditioner Loads

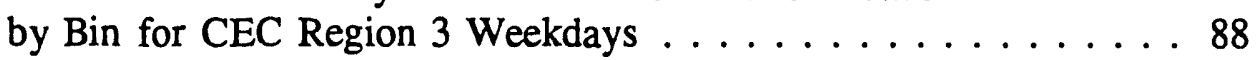

Figure 7-4. Distribution of Maximurn Hourly Load for Two Binning

Schemes on AVGDRY, CEC Region 3 Weekday . . . . . . . 89

Figure 7-5. Distribution of Maximum Hourly Load for Binning

MXDRY, THISUM, and CDD80SM for CEC Region $3 \ldots \ldots 90$

Figure B-1. Central Air Conditioner Load Profiles by Bin for PG\&E Zone R 105

Figure B-2. Central Air Conditioner Load Profiles by Bin for PG\&E Zone R 106

Figure B-3. Central Air Conditioner Load Profiles by Bin for PG\&E Zone R 107

Figure B-4. Central Air Conditioner Load Profiles by Bin for PG\&E Zone S . 108

Figure B-5. Central Air Conditioner Load Profiles by Bin for PG\&E Zone S . 109

Figure B-6. Central Air Conditioner Load Profiles by Bin for PG\&E Zone S . 110

Figure B-7. Central Air Conditioner Load Profiles by Bin for PG\&E Zone X 111

Figure B-8. Central Air Conditioner Load Profiles by Bin for PG\&E Zone X 112

Figure B-9. Central Air Conditioner Load Profiles by Bin for PG\&E Zone X 113

Figure B-10. Central Air Conditioner Load Profiles by Bin for CEC Region 2 . 114

Figure B-11. Central Air Conditioner Load Profiles by Bin for CEC Region 2 . 115

Figure B-12. Central Air Conditioner Load Profiles by Bin for CEC Region 2 . 116

Figure B-13. Central Air Conditioner Load Profiles by Bin for CEC Region 3 . 117

Figure B-14. Central Air Conditioner Load Profiles by Bin for CEC Region 3 . 118 
Figure B-15. Central Air Conditioner Load Profiles by Bin for CEC Region 3 . 119

Figure B-16. Central Air Conditioner Load Profiles by Bin for CEC Region 4 . 120

Figure B-17. Central Air Conditioner Load Profiles by Bin for CEC Region 4 . 121

Figure B-18. Central Air Conditioner Load Profiles by Bin for CEC Region 4 . 122

Figure C-1. Distribution of Season-Specific Model Residuals (obs.-pred.) for CEC Region 2 . . . . . . . . . . . . . 123

Figure C-2. Distribution of Season-Specific Model Residuals (obs.-pred.) for CEC Region 3 . . . . . . . . . . . . . . . 124

Figure C-3. Distribution of Season-Specific Model Residuals (obs.-pred.) for CEC Region 4 . . . . . . . . . . . 125

Figure C-4. Distribution of Season-Specific Model Residuals (obs.-pred.) for PG\&E Zone R . . . . . . . . . . . . . 126

Figure C-5. Distribution of Season-Specific Model Residuals (obs.-pred.) for PG\&E Zone S . . . . . . . . . . . . . . . 127

Figure C-6. Distribution of Season-Specific Model Residuals

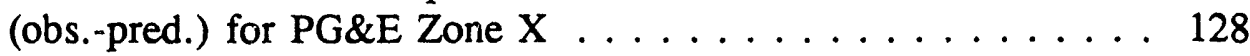


End-use electricity demand forecasts play a critical role in resource planning approaches that actively consider both supply- and demand-side options to meet customer energy service needs. Yet, in order to forecast peak demands by end use, utility and state planners have had to rely on both simulated and borrowed end-use and class load research data. This reliance has introduced additional uncertainty into an already complicated resource planning process, as questions arise regarding the veracity of these inputs. The data now available from recent end-use metering projects holds the promise of reducing these uncertainties and thereby improving the planning process and its outcomes.

This report summarizes findings from a unique project to improve the end-use electricity load shape and peak demand forecasts made by the Pacific Gas and Electric Company (PG\&E) and the California Energy Commission (CEC). First, the direct incorporation of end-use metered data into electricity demand forecasting models is a new approach that has only been made possible by recent end-use metering projects. Second, and perhaps more importantly, the joint-sponsorship of this analysis has led to the development of consistent sets of forecasting model inputs. That is, the ability to use a common data base and similar data treatment conventions for some of the forecasting inputs frees forecasters to concentrate on those differences (between their competing forecasts) that stem from real differences of opinion, rather than differences that can be readily resolved with better data.

The focus of the analysis is residential space cooling, which represents a large and growing demand in the PG\&E service territory. Using five years of end-use metered, central air conditioner data collected by PG\&E from over 300 residences, we developed consistent sets of new inputs for both PG\&E's and CEC's end-use load shape forecasting models. We compared the performance of the new inputs both to the inputs previously used by PG\&E and CEC, and to a second set of new inputs developed to take advantage of a recently added modeling option to the forecasting model. The testing criteria included ability to forecast total daily energy use, daily peak demand, and demand at 4 P.M. (the most frequent hour of PG\&E's system peak demand). We also tested the new inputs with the weather data used by PG\&E and CEC in preparing their forecasts.

We find that the new inputs developed in this project perform significantly better than previous inputs used by CEC and generally better than previous inputs used by PG\&E. We also find that, while the use of the new forecasting option did sometimes lead to modest improvements, the additional effort required to take advantage of this option in forecasting future data is significant and may not be justified by the results. 
In testing the new inputs with the same weather data files used by PG\&E and CEC in their forecasts, we verified that the magnitude of potential implementation issues raised by PG\&E and CEC was small and made a simple recommendation to address them.

In the course of the analysis, the project staff, PG\&E, and CEC identified several issues of interest outside the primary focus of the main body of research. We conducted exploratory investigations for several of these issues. Our findings are summarized as follows:

1. We considered explicit inclusion of a "heat-storm" variable in models to allocate annual energy use to days of the year, but concluded that inclusion is not warranted, at this time, without a more systematic examination of related problems associated with forecasting energy use on "peak" days.

2. We examined metered data collected for heat pumps (which rely on the same technology as central air conditioners, but also provide cooling), but conclude that would be premature to simply add cooling loads from the small sample of heat pump compressors to those from the much larger sample of central air conditioners without additional information on the residences and equipment being monitored.

3. We developed a separate set of daily energy models for room air conditioners, which confirmed our intuitions regarding differences between patterns of central air conditioner versus room air conditioner energy use. However, the comparatively smaller sample of room air conditioners precludes us from drawing definitive conclusions as to the ultimate significance these differences.

4. We examined data from several weather stations and concluded that the weather station currently used by CEC to model central air conditioning energy use in the transitional climate region 4 performs better than the two alternatives considered.

5. We developed a procedure for systematically evaluating the specification of binned hourly load shapes and confirmed the appropriateness of current reliance on dry bulb temperature for this purpose.

6. We developed separate daily energy models specifically to forecast energy use on peak days, which indeed perform better than models developed to forecast energy use on all days. Nevertheless, we reserve judgement on their usefulness pending the outcome of a much broader discussion on how the 
increased effort to take advantage of additional sub-models (such as this one) should be balanced by the other forecasting requirements for load shape models.

Our analyses jointly suggest that the need for additional research must be predicated on a systematic assessment of competing load shape forecasting objectives (such as forecasting system peak, hourly load shapes for 12 typical weeks, minimum load conditions, etc.) in light of the resource constraints faced by the forecasting process. At that time, the following enhancements should be considered:

1. More systematic examination of daily load shape bins for possible respecification based on other forecasting objectives; our exploratory analysis has led to the development of a framework for this activity.

2. Continued refinement and integration of separate peak day models into the overall load shape forecasting process; we have demonstrated the potential value of this approach.

3. Sample weight development based on consideration of current forecasting conventions for the specification of the population (e.g., regions versus rate classes); all analyses undertaken to date have been based on un-weighted data.

4. Forecasting weather data file development based on capturing specific meteorological phenomena of interest for forecasting (such as heat storms). 


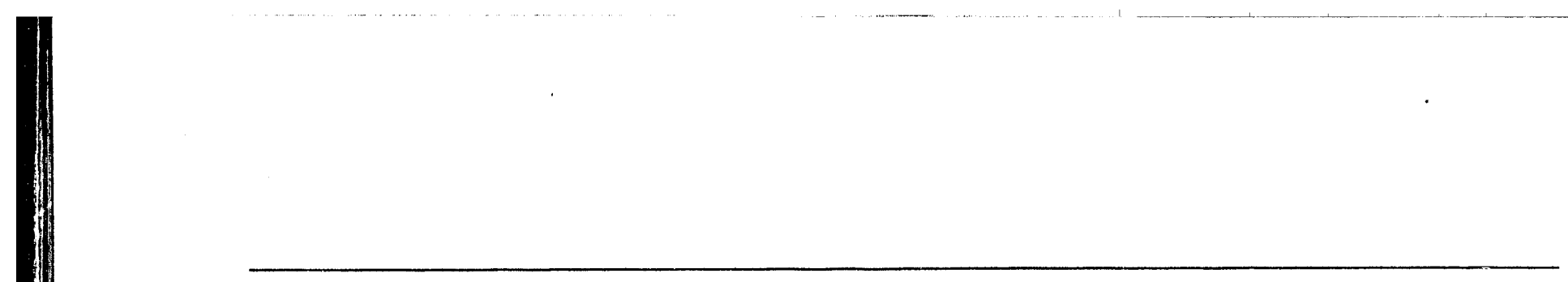

$$
\text { xii }
$$




\section{Introduction}

End-use electricity demand forecasts play a critical role in resource planning approaches that actively consider both supply- and demand-side options to meet customer energy service needs. Yet in order to forecast peak demands by end use, utility and state planners have had to rely on both simulated and borrowed end-use data and on class load research data. This reliance has introduced additional uncertainty into an already complicated resource planning process, as questions arise regarding the veracity of these inputs. The data now available from recent end-use metering projects holds the promise of reducing these uncertainties and thereby improving the planning process and its outcomes.

This report summarizes findings from a unique project to improve the end-use electricity load shape and peak demand forecasts made by the Pacific Gas and Electric Company (PG\&E) and the California Energy Commission (CEC). First, the direct incorporation of end-use metered data into electricity demand forecasting models is a new approach that has only been made possible by recent end-use metering projects. Second, and perhaps more importantly, the joint-sponsorship of this analysis has led to the development of a common set of forecasting model inputs. That is, the ability to use a common (but not identical) data set for some of the forecasting inputs frees forecasters to concentrate on those differences (between their competing forecasts) that stem from real differences of opinion, rather than differences that can be readily resolved with better data.

This report is the second stemming from this jointly-sponsored approach for developing improved residential electricity demand forecasts through consistent analyses of a common set of end-use metered data. The first report documented procedural efforts to prepare the end-use data for analysis and the development of forecasting model inputs for an older forecasting model used by CEC (Eto and Moezzi 1992). Since that time, CEC has adopted the same forecasting model used by PG\&E (although, important differences remain between PG\&E's and CEC's model specifications).

This report summarizes the development of two alternative approaches for this now commonly used model and an evaluation of these alternatives (and those summarized in the first report) along with the inputs currently used by PG\&E and CEC. The focus of the analysis is residential space cooling, which represents a large and growing demand in the PG\&E service territory. In Chapter 2, we review the background for the project by describing the end-use metered data being analyzed, the forecasting model for which the new inputs are being developed, and the differences in the use of the models by PG\&E and CEC. In Chapters 3 and 4, we describe aspects of the development of inputs 
for one approach to using the forecasting model. In this approach, a forecast of annual electricity use for space cooling is first allocated to each day of the year (Chapter 3) and then separately allocated to the hours of the day (Chapter 4). In Chapter 5, we describe the development of inputs for a recently available alternative to this approach in which the annual forecast is allocated directly to each hour of the year. In Chapter 6, we describe our evaluation of all of the approaches.

Chapter 7 contains the results of exploratory analyses developed in response to the interests of PG\&E and CEC. These analyses consider issues outside the scope of the main research agenda, but which might influence future forecasting enhancements. The issues include: 1) the incremental improvement in accuracy resulting from the explicit inclusion of a measure of heat storms $\mathrm{s}^{1}$ as an explanatory variable for the allocation of annual electricity use to the days of the year; 2) the energy use patterns of heat pumps, which employ the same technology as central air conditioners, but also provide heating; 3) the energy use patterns of room air conditioners, which also employ the same technology as central air conditioners, but whose application is more localized within a residence; 4) the impact of using data from different weather sites to explain space cooling energy use in one of PG\&E's more temperate regions; 5) a systematic examination of the current method used to specify binned load shapes; and 6) preliminary findings from a direct examination of the energy use characteristics of peak days versus average days.

Chapter 8 draws upon the analyses to suggest additional areas of work identified in the course of our research. Appendix A summarizes data handling conventions. Appendices $B$ and $C$ contain additional graphical summaries of project results from Chapters 4 and 6 , respectively. Appendix D comments on the use of our research in forecasts made with the HELM model.

\footnotetext{
${ }^{1}$ A heat storm is a multi-day episode of high ambient temperature. It has been suggested that the cumulative effect of several hot days increases the use of electricity for space cooling at a given temperature relative to the electricity used on an identically hot day that has not been preceded by hot days.
} 


\section{Background}

In the first phase of this project, we analyzed end-use metered data collected by PG\&E to develop new inputs for the peak demand forecasting model used historically by CEC (see Eto and Moezzi 1992). Since that time, CEC has adopted the peak demand model used by PG\&E. The goal of this second phase of the project is to use PG\&E's space cooling data to develop inputs for this second model and to compare these inputs to those previously used by PG\&E and CEC for this purpose. In this chapter, we review the data to be used in the analysis, summarize the basic methods employed by both the now commonly used PG\&E and CEC model and the older CEC model, and contrast the differences between PG\&E's and CEC's use of the common model.

\subsection{The PG\&E Appliance Metering Project}

PG\&E's Appliance Metering Project (AMP) was the first large-scale end-use metering project in California (Brodsky and McNicoll 1987). Since 1983, more than 700 singlefamily, owner-occupied residences have been continuously metered. In designing the project, PG\&E was particularly interested in improving its understanding of the contribution of space cooling energy use to system loads. As a result, the geographic distribution of metered households is concentrated in the hot central valley of California where the demand for cooling is greatest.

For each household, two appliances were metered in addition to total household load. In the entire sample, a total of sixteen different appliance types were metered. In this study, we analyze only the data collected on central air conditioning energy use from about 350 households, although exploratory work reported in Chapter 7 considers limited aspects of the data collected on heat pump compressors and room air-conditioning energy use. $^{2}$

PG\&E provided LBL with a total of five years of hourly data collected between 1985 and 1989. PG\&E replaced all information that might identify individual customers with a seven digit code that identified the households across data sets.

\footnotetext{
${ }^{2}$ Other end uses were also examined in the first phase of the project incliunng refrigerators, clothes washing, and clothes drying. See Eto and Moezzi 1992. These results were used to develop new inputs for the older in-house CEC model; PG\&E, in its application of HELM, does not consider these end uses explicitly.
} 
PG\&E has also developed weights to make the sample more representative of the entire residential class, and to account for the stratified nature of the AMP sample. Through discussion with the project sponsors, which considered the fact that there are differences between PG\&E and CEC application of the data that are not reflected in the development of sample weights, that sample weights were not developed for the 1985 and 1986 data, and that the focus of the LBL analysis is on load shape, not energy use, it was agreed that these weights would not be used in the present analysis. Therefore, with the exception of the weighted mean unit energy consumption or UEC presented in this chapter, the results presented in this report were developed through unweighted analyses of the data. Our unweighted analyses are theoretically reflective only of loads for those single-family owner-occupied residences metered by the project; furthermore, for these loads, we cannot determine what biases may exist as a result of the process used to select participants for the project.

\subsection{Electricity Load Shape Forecasting Models}

Current hourly electricity load shape and peak demand models are essentially postprocessors for end-use forecasting models, which separately generate annual forecasts of energy use. In this modeling framework, equipment purchase and energy use decisions, stock turnover, and other economic and demographic factors are treated as influences primarily on annual energy use. The goal of the load shape and peak demand model is simply to allocate the estimated annual total load to the hours of the year.

PG\&E and CEC currently use a load shape forecasting model called HELM, developed by ICF, Inc. for the Electric Power Research Institute (ICF Resources Inc. 1992). The model is very flexible; the user must specify the number of end uses to be forecast as well as the method for generation of up to an 8,760-hour forecast of energy use. The model supports two alternative approaches for generating this forecast.

In its standard two-stage application, the user first defines hourly load shapes for a limited number of day-types and second, assigns day-types to all the days in a calendar year for each end use and forecast year. The model then distributes annual energy use (typically, form an end-use forecasting model) to the hours of the year, using previously defined allocation factors. For weather-sensitive end uses, such as cooling, the allocation procedure may be based on measures of climatic variables, such as a three-day weighted average of mean daily temperature. In this case, the model also requires a daily weather file for the year. Daily energy use is allocated to the hours of the day using normalized 
load shapes. ${ }^{3}$ The choice of normalized load shape can be specified as a function of weather variables.

In the latest version of HELM 2.0, the user may bypass the two-stage process by specifying load shapes directly on an hourly basis. Choice of this approach replaces both the allocation of annual energy to days of the year and the selection of a normalized daily load shape.

Previously, CEC used a peak demand model that was developed in-house in the late 1970's (Jaske and Paige 1979). The CEC model is more structured than HELM because it was designed for use in conjunction with detailed end-use annual energy forecasting models that were also developed by CEC. For the residential sector, the CEC model requires annual energy forecasts for 14 non-space conditioning and five space conditioning end uses for each geographic region considered. In the past, the CEC model has been used primarily to produce system peak day load forecasts although the model is, in principle, capable of producing forecasts for non-peak days.

The CEC model, like HELM, allocates a forecast of annual energy use to the hours of the year in two steps. For space cooling, a forecast of annual space cooling energy use is first allocated to daily energy use using weather data: CEC uses a three-day weighted average of degree-days. The degree-days are based on a combined dry- and wet-bulb temperature variable called a temperature-humidity index or $\mathrm{THI} .{ }^{4}$ Unlike HELM, the CEC model does not spread daily energy use to the hours of the day using a fixed load shape. Instead, daily energy use is distributed to the hours of the day as a function of time of day and THI (for cooling) or dry-bulb temperature (for heating) in what CEC refers to as a time-temperature matrix.

\subsection{PG\&E and CEC Peak Demand Forecasting Model Specifications}

Although PG\&E and CEC now use the same HELM modeling framework, their use of the model differs in several important ways. PG\&E defines seven separate end uses for the residential sector: three separate space cooling and three non space cooling end uses corresponding to three geographic zones within the company's service territory, and a

\footnotetext{
${ }^{3}$ The sum of hourly loads in one day's normalized load shape is 1 . The load in each hour, therefore, represents the proportion of total daily energy use in that hour.

${ }^{4} \mathrm{THI}$ is defined as follow: $\mathrm{THI}=15+0.4 *(\mathrm{DBT}+\mathrm{WBT})$, where $\mathrm{DBT}=$ dry-bulb temperature and $\mathrm{WBT}=$ wet-bulb temperature.
} 
single combined end use for a fourth geographic zone (Pacific Gas and Electric Company 1991). Figure 2-1 indicates these zones.

To forecast system peak demands for the PG\&E planning area, the CEC model produces separate forecasts for five geographic regions (California Energy Commission (CEC) 1991), as shown in Figure 2-2. Within each region, four space cooling (single-family and multi-family central and room air conditioning), six space heating, and ten non-space conditioning end uses are separately forecast.

Our approach to recognizing differences in the ways in which PG\&E and CEC develop forecasts for the PG\&E service territory is to prepare separate sets of inputs from the common data set of end-use metered data. Specifically, individual metered data are first aggregated to the appropriate sub-service territory zone (in the case of PG\&E) or region (in the case of $\mathrm{CEC}$ ). The resulting zonal or regional load shapes are then analyzed using common procedures to develop a consistent (yet separate) set of inputs for PG\&E's and CEC's application of the HELM model.

Descriptive statistics of the data analyzed in this project are summarized in Table 2-1 and Table 2-2. Each table reports unweighted and weighted UECs (in $\mathrm{kWh} /$ year), load factors, and coincidence factors by the separate forecasting zones or regions used by PG\&E and CEC in their forecasts.

Table 2-1 and Table 2-2 also show the geographic distribution of cooling appliances by PG\&E zone and by CEC region. Several other forecasting zones and regions are defined by PG\&E and CEC for forecasting purposes but have little or no cooling requirements and, accordingly, have very low central air conditioner saturations. As a result, few households were metered. ${ }^{5}$ As a result, we did not conduct analyses for these zones and regions.

\footnotetext{
${ }^{5}$ PG\&E's Zone T contained only 1 metered central air conditioners and CEC's Regions 1 and 5 contained only 3 and 6 metered central air conditioners, respectively.
} 
Table 2-1. Annual UEC Load Factor (LF), and Coincidence Factor (CF) for Central Air Conditioning, PG\&E Zones

\begin{tabular}{|c|c|c|c|c|c|c|}
\hline APPLIANCE & 1985 & 1986 & 1987 & 1988 & 1989 & $\begin{array}{l}\text { annual } \\
\text { average }\end{array}$ \\
\hline \multicolumn{7}{|l|}{ All Zones } \\
\hline mean UEC & 1,254 & 1,112 & 1,424 & 1,607 & 1,094 & 1,294 \\
\hline wt.mean UEC & $n / a$ & $n / a$ & 1,283 & 1,429 & 996 & $n / a$ \\
\hline$n$ & 288 & 267 & 337 & 318 & 332 & 308 \\
\hline \multicolumn{7}{|l|}{ Zone R } \\
\hline mean UEC & 1,802 & 1,700 & 2,002 & 2,294 & 1,684 & 1,905 \\
\hline wt.mean UEC & $n / a$ & $n / a$ & 1,653 & 1,911 & 1,519 & $n / a$ \\
\hline st.dev. UEC & 565 & 543 & 718 & 701 & 601 & 678 \\
\hline$n$ & 86 & 82 & 117 & 113 & 111 & 102 \\
\hline load factor & 0.063 & 0.031 & 0.073 & 0.074 & 0.067 & $n / a$ \\
\hline factor & 0.92 & 0.94 & 0.83 & 0.91 & 0.90 & $n / a$ \\
\hline \multicolumn{7}{|l|}{ Zone S } \\
\hline mean UEC & 1,277 & 1,188 & 1,367 & 1,538 & 1,018 & 1,265 \\
\hline wt.mean UEC & & $n / a$ & 1,270 & 1,380 & 895 & $n / a$ \\
\hline st.dev. UEC & 553 & 495 & 576 & 580 & 479 & 438 \\
\hline$n$ & 116 & 102 & 132 & 118 & 123 & 118 \\
\hline load factor & 0.048 & 0.049 & 0.057 & 0.054 & 0.042 & $\mathrm{n} / \mathrm{a}$ \\
\hline factor & 0.91 & 0.92 & 0.89 & 0.93 & 0.91 & $n / a$ \\
\hline \multicolumn{7}{|l|}{ Zone X } \\
\hline mean UEC & 682 & 497 & 709 & 790 & 521 & 629 \\
\hline wt.mean UEC & $n / a$ & $n / a$ & 717 & 753 & 500 & $n / a$ \\
\hline st.dev. UEC & 249 & 239 & 737 & 443 & 254 & - \\
\hline$n$ & & 80 & 86 & 86 & 97 & 86 \\
\hline load factor & 0.029 & 0.029 & 0.038 & 0.033 & 0.028 & $n / a$ \\
\hline factor & 0.90 & 0.91 & 0.92 & 0.92 & 0.90 & $n / a$ \\
\hline \multicolumn{7}{|c|}{$\begin{array}{l}\text { For a description of PG\&E zones } R, S \text { and } X \text {, please refer to Figure } 2-1 . \text { Zone } T \text { is the coastal regions } \\
\text { where air conditioning use is relatively negligible. }\end{array}$} \\
\hline
\end{tabular}


Table 2-2. Annual UEC, Load Factor (LF), and Coincidence Factor (CF) for Central Air Conditioning, CEC Regions

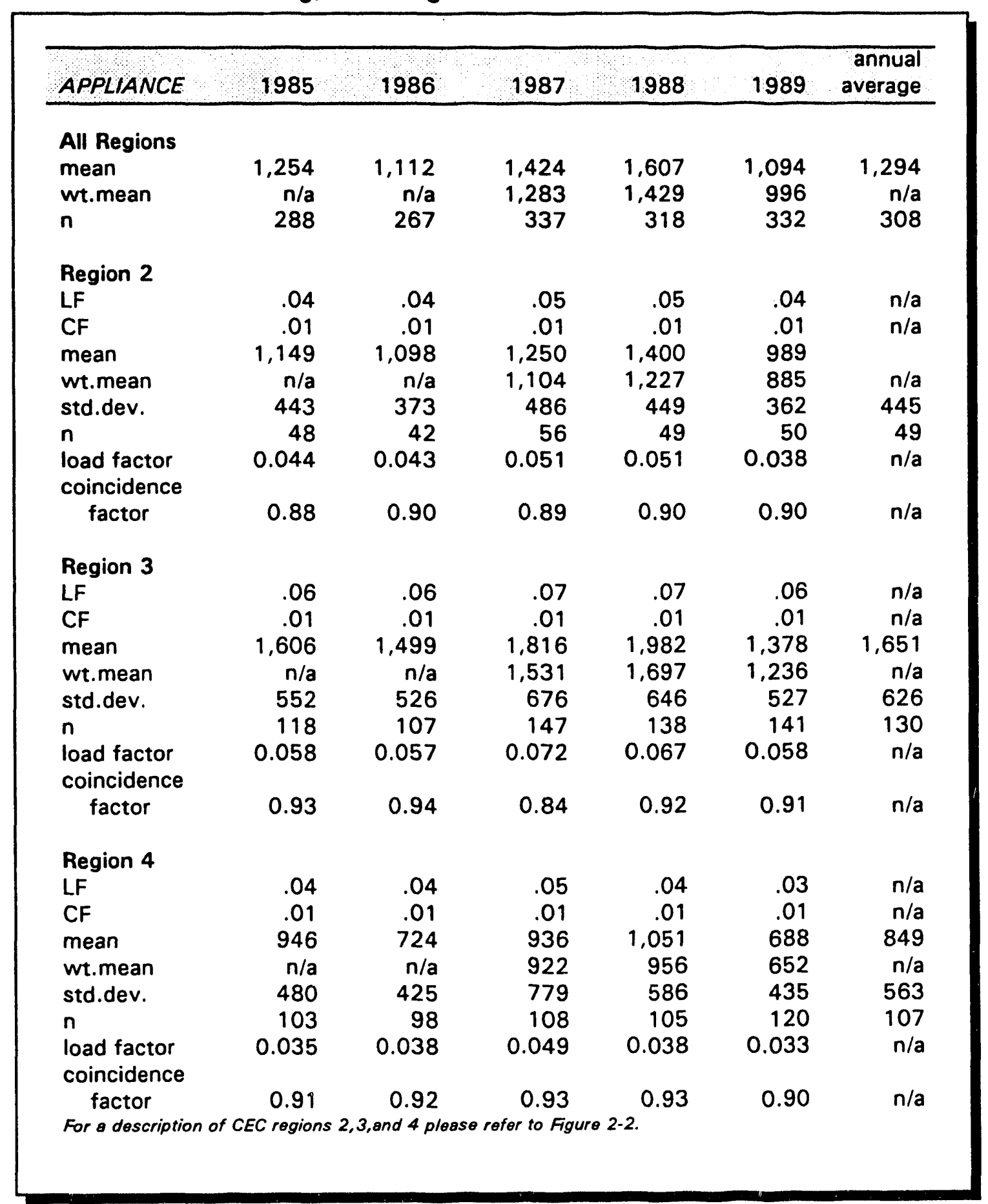




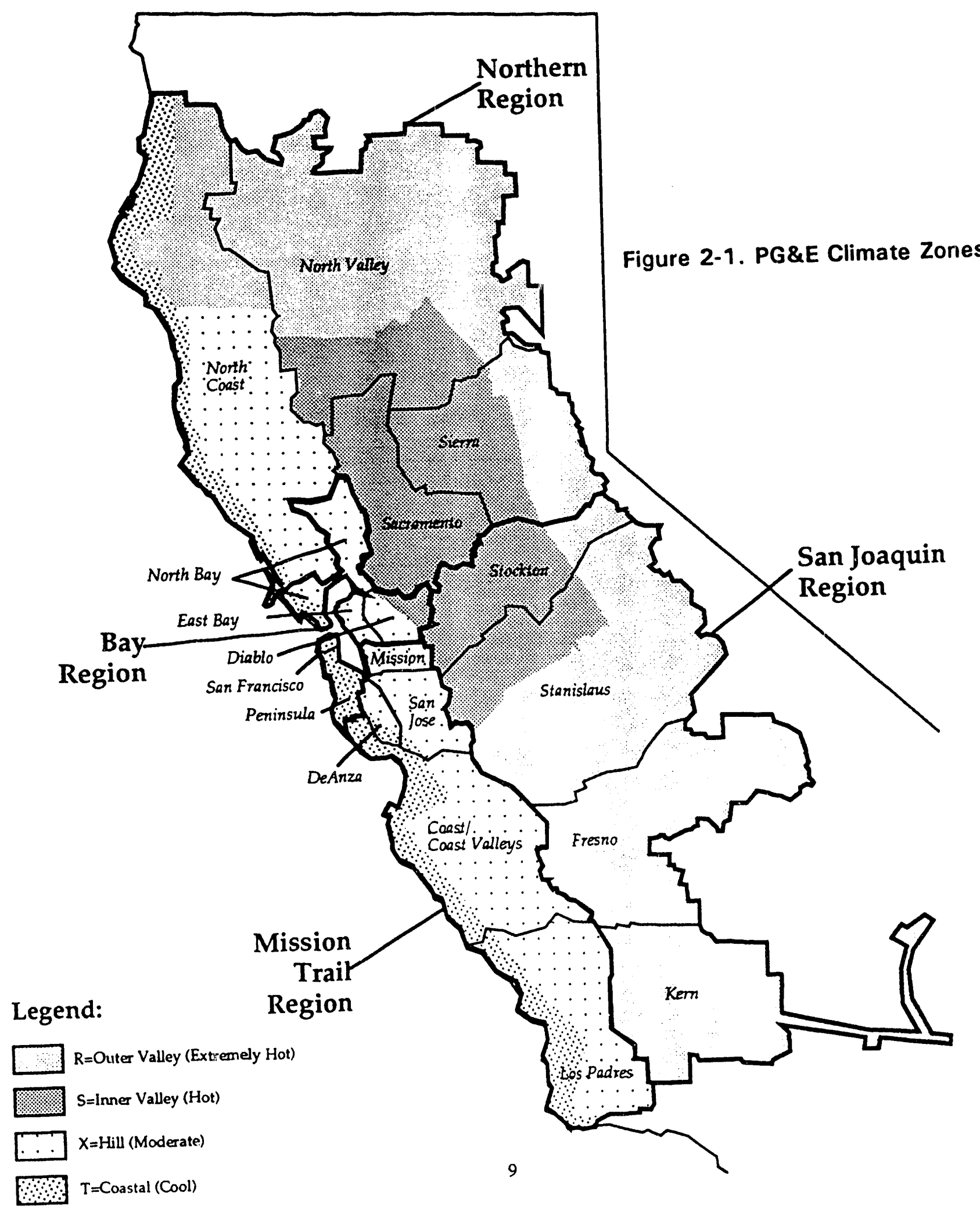




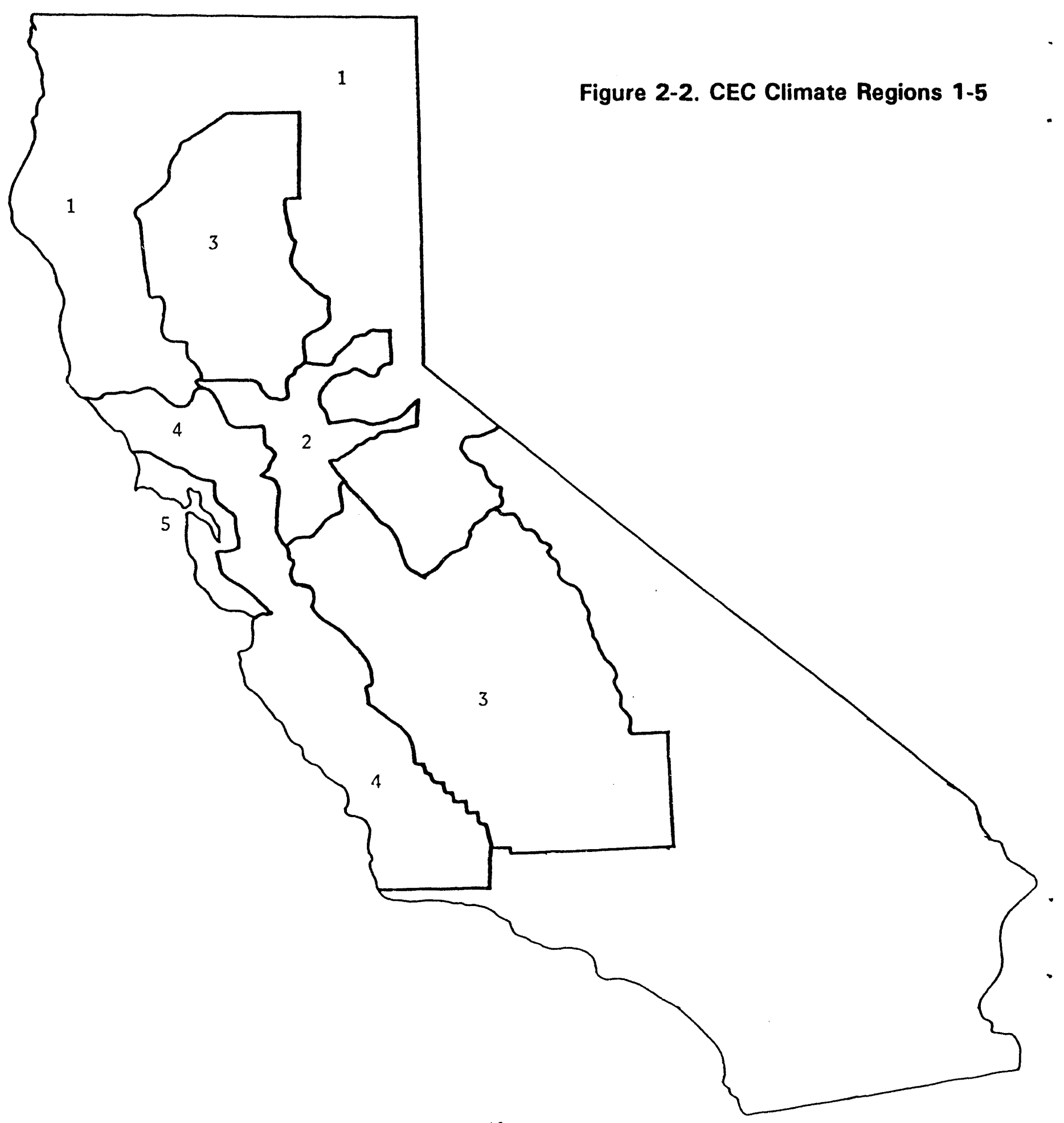




\section{Daily Energy Model}

The principal forecasting method offered by HELM is to forecast hourly energy in two stages. First, HELM allocates estimates of annual energy use to each day of the year. Second, the model allocates the resulting daily energy estimates to hours of the day. For weather-sensitive end uses, such as air conditioning, the allocation of annual to daily energy is based on daily values of weather variables according to a "weather response function" (WRF), and on calendric characteristics such as season and day of the week. This is the method PG\&E and CEC have historically used to produce their forecast. In this chapter we describe how we used the PG\&E Appliance Metering Project (AMP) central air conditioning data to develop specifications for the annual-to-daily energy allocation component of this HELM method. ${ }^{6}$ The bases for these specifications are season-specific linear regression models which use weather data measured at sites in the PG\&E service territory to model regional or zonal daily averages of AMP central air conditioner loads.

In this chapter we first discuss aspects of the load and corresponding weather data, as well as the spatial and temporal aggregation of these data for the purposes of daily load modelling. We then discuss the procedures used to develop daily energy regression models for use in HELM and describe some of our results. The second component of this forecasting method, that of allocating daily energy to hours of the day, is discussed in Chapter 4. Model evaluation issues are presented in Chapter 6.

\subsection{Load Data}

Many households in the AMP sample have dual-purpose central space conditioning systems that combine electric central cooling with gas central heating. For some of these households, the metering equipment used records the demands of both the compressor used for cooling and the circulation system used for both heating and cooling. Thus, not all recorded central air conditioner loads can be attributed to the cooling end use: a small

\footnotetext{
${ }^{6}$ As described in Chapter 2, the AMP sample contains data for three types of appliances associated with the cooling end use: central air conditioner, room air conditioner, and heat pump compressor. In this chapter we model central air conditioner data only. Heat pump compressors employ the same technology for cooling as do central air conditioners, but modeling heat pump compressor data is complicated by the fact that heat pump compressors are used for heating as well as cooling. Although we did not develop regression models using heat pump compressor data, we discuss some aspects of these data in Chapter 7. We did develop regression models for room air conditioner loads for two zones and one region; these results are also presented in Chapter 7 .
} 
fraction of the loads may be for heating circulation. PG\&E Load Research estimates that the average circulation system uses about $1.2 \mathrm{kWh}$ per day during the heating season (Brodsky and McNicoll 1987). CEC considers heating ventilation loads as part of the heating, rather than cooling, end use.

To eliminate non-cooling electricity consumption, we developed two rules for treating observed values. We set the cooling end use load for central air conditioner to zero for the Winter season (November to March), thus excluding fan loads for heating, as well as any use of the central air conditioner for cooling which might have occurred during these five months. For the remaining months, we modeled cooling load as zero for all days in which the average daily dry-bulb temperature fell below a specified threshold based on previous PG\&E research $(62.2,66.1$, and 58.6 degrees for PG\&E Zones $R$, $\mathrm{S}$, and X respectively, and 62.2, 66.1, and 58.6 degrees for CEC Regions 2, 3, and 4, respectively). ${ }^{7}$

As a result, the annual energy use modeled as cooling is less than the central air conditioner UEC, because the former excludes loads in Winter and loads occurring on cool days, whereas the latter includes all recorded central air conditioner loads. In the AMP sample the average percentage of annual UEC occurring in November through February is $10.2,12.3$, and 7.9 percent for PG\&E Zones R, S, and X respectively. ${ }^{8}$

\subsection{Weather Station Assignment and Explanatory Variable Definition}

As described in Chapter 2, we used two separate sets of geographic aggregation for our analyses, one by PG\&E Zone (zones R, S, and X), and one by CEC Region (regions 2, 3 , and 4), with each metered residence assigned to one zone and to one region. Each PG\&E zone is associated with a weather station maintained by PG\&E, and each CEC region is associated with a National Oceanic and Atmospheric Administration (NOAA) weather station, according to assignments give by $P G \& E$ and $C E C$, respectively. PG\&E weather stations at Fresno, Sacramento, and San Ramon are used for zones R, S, and X, respectively, and NOAA weather stations at Fresno, Sacramento, and San Jose/Sunnyvale

\footnotetext{
${ }^{7}$ Our examination of data for individual residences indicates that some cooling loads may occur on these days. However, we did not model them, as they contributed negligible loads to the region- or zone-wide average load shape. These loads might be accounted for by computing seasonal average load (instead of 0 ) or as a miscellaneous or heating end use.

${ }^{8}$ We noticed that a relatively high percentage of the higher winter loads assigned to the central air conditioner end use occurred in late December.
} 
are used for CEC regions 2, 3, and 4, respectively. See Table 3-1. The correspondence between the two sets of geographic aggregations is discussed in Appendix A.

Half-hourly measurements of dry-bulb temperature and relative humidity are recorded for each PG\&E weather station. Hourly measurements of dry-bulb temperature, wet-bulb temperature, wind speed, cloud cover, and a number of other meteorological characteristics are recorded at each NOAA station. From these primary data sets we developed a set of secondary weather variables to be used as potential explanatory variables in daily energy models. For the PG\&E stations, we based these weather variables on hourly averages of measured half-hourly dry-bulb temperature and of relative humidity. For the NOAA stations, we based these weather variables on measured hourly dry-bulb and wet-bulb temperature.

Table 3-2 lists the set of daily weather variables we derived from the hourly NOAA and PG\&E weather data, and provides definitions for each variable. The variables include average dry-bulb temperature, maximum and minimum hourly dry-bulb temperature, (MXDRY and MNDRY) degree-days of the temperature-humidity index base $68 \circ \mathrm{F}$ (THIDD; see also Appendix A), and a series of daily sums of cooling degree hours using temperature bases from $75 \circ \mathrm{F}$ to $95 \circ \mathrm{F}$ in five degree increments. For our regression models, we considered as possible covariates not only the value of each variable on the day modeled, but the value of the variables one and two days previous. Recent weather variability may also influence cooling demand. We did not examine this phenomena in detail, but attempted to capture some of this effect in the variable TVARMX, defined as the variance of the maximum dry-bulb temperature over the three days up to and including the day modeled, ${ }^{9}$ and the components of another measurement of temperature variables developed by Ignelzi and Way (1988), and subsequently used by Kristov (1991); we call these variables SCE1 (MNDRY * MXDRY1) and SCE2 (MNDRY1 * MXDRY2). ${ }^{10}$

\footnotetext{
${ }^{9}$ Ignelzi and Way (1988) instead use a temperature variance variable proportional to TVARMAX * MXDRY.

${ }^{10}$ In the CEC-sponsored California Weather Index Development project, Kristov (1991) addressed the use of various meteorological variables for use in modeling weather-sensitive loads. Kristov (1991) describes investigations of several weather indices developed to support energy load modeling applicable to California, and shows results of tests of these indices for modeling SMUD and PG\&E system data, as well as 1989 AMP average central air conditioner load data, for both daily and hourly load modeling. The primary meteorological data used were NOAA measurements of dry-bulb temperature, dew point, wind speed, and cloud cover; secondary measures included THI, cooling degree days, and variables based on principal components analyses of the primary variables.
} 


\subsection{Calendric Aggregation}

We disaggregated data into four seasons: Winter (November through March), Spring (April and May), Summer (June through August), and Fall (September and October), based on an examination of the monthly distributions of daily average temperature. ${ }^{11}$ We will use these same seasonal definitions for the derivation of load shape libraries (discussed in Chapter 4). We investigated, but did not use, day-of-week or day types (Weekday vs. Weekend/Holiday) to model daily energy, since we detected only very small or nonexistent effects. However, while none of our final models for daily load use day type as an explanatory variable, we did develop distinct load shape libraries for Weekday and for Weekend (see Chapter 4), which is consistent with the current PG\&E practice of developing daily weather response functions irrespective of day type. ${ }^{12}$

\subsection{Linear Regression Models}

In tr past, PG\&E modeled cooling loads as a functior. of linear and quadratic terms of a three-day weighted average dry bulb temperature (WAVGDRY) where for day $i$ :

WAVGDRY $[i]=0.5 *$ AVGDRY $[i]+0.3 *$ AVGDRY $[i-1]+0.1 *$ AVGDRY $[i-2]$,

where AVGDRY = average dry-bulb temperature,

so that the regression model is:

$$
\operatorname{SUMLOAD}[i]=\alpha+\beta * \text { WAVGDRY }[i]+\gamma^{*} \text { WAVGDRY }[i]^{2}+\text { error, }
$$

where $\alpha, \beta$ and $\gamma$ are coefficients estimated using ordinary least squared regression on total daily energy use, SUMLOAD.

To revise these regression models, we used an automatic variable selection procedure known as stepwise regression to help select a "good" (in terms of variance explained by the model; that is, r-squared) linear model for SUMLOAD, using as potential

$"$ We also estimated models for the CEC regions for an Extended uummer season defined as the four month period June through September. This Extended Summer season corresponds to a definition used in some past work, e.g., PG\&E's 1989 WRFs.

12 In previous forecasts, PG\&E has used two day types (Weekday and Weekend) and one season, defined as the twelve months of the year, with the load shapes libraries and weather response functions derived on the basis on load data for summer (June through September) months only. 
explanatory variables the covariates listed in Table 3-2. To initialize the stepwise procedure, one specifies a response variable (here SUMLOAD), a set of variables to be used as potential explanatory variables in the regression model (here the daily weather variables described in Table 3-2), and an "F-to-enter" and "F-to-remove" which specify the degree of explanatory capability an individual covariate must meet to be entered or omitted from the regression equation at any step. Variables are added one at a time to the current model. After each addition, the F-value of all variables in the model is evaluated; any variable with an F-statistic less than the specified "F-to-remove" value is omitted. The stepwise procedure ends when all variables in the model have F-statistics greater than F-to-remove value, and all variables outside the model have F-statistics lower than the F-to-enter value. We used an F-value of 0.15 for both F-to-enter and Fto-remove. ${ }^{13}$ In developing the final HELM input files, we reduced the dimensionality of most of the models resulting from the stepwise regression procedure, as discussed below.

While the stepwise procedure results in sets of covariates which have relatively high explanatory capability, results must be interpreted with caution: (1) the procedure is not optimal, in that it does not necessarily result in the subset of a given size which has the highest possible $\mathrm{r}$-squared (that is, for a fixed model dimensionality, the procedure does not necessarily optimize the set of explanatory variables in terms of maximizing explanatory capability); and (2) many alternative sets of variables may have nearly the same $r$-squared as the final subset resulting from the stepwise procedure. Still, the procecure is useful in comparing sets of variables to be used in predictive models.

Because these regression equations were to be used in HELM, we considered the following practical issues in our model specifications: (1) HELM PC 2.0 limits the number of weather variables which can be specified in models for weather-sensitive end uses to six,${ }^{14}$ and (2) although it is possible to specify different sets of weather variables for each season in the model for each season, it is awkward to do so. For these reasons, we constrained the number of variables in the regression model specified in the HELM input files to six and used the same variables for each season. To select these variables, we examined the variables in the season-specific models, and the marginal $r$-squared contribution of each variable according to the stepwise procedure. We first chose those variables ranked highest in terms of marginal r-squared for the Summer regression models and then augmented these basic models according to the variables selected for the Fall and Spring Seasons.

\footnotetext{
${ }^{13}$ The SAS default value for F-to-enter and F-to-remove is 0.15 .

${ }^{14}$ Quadratic and higher powers of each of these six variables may be used, in addition to the linear term.
} 
Tables 3-3 and 3-4 shows the variables included in the resulting HELM regression models for CEC regions 2, 3, and 4 and for PG\&E zones R,S, and X, respectively. These represent the WRFs used in HELM, which were derived from a stepwise linear regression procedure using the full set of variable listed in the left column. Each column gives the regression coefficients for all variables included in the WRF. The regression coefficients are provided for comparative purposes only; please refer to the HELM input files for higher precision in regression coefficient values.

The tables also show r-squared for these models, and, for comparison, the r-squared from models achieved by the subset stepwise procedure. With the caveat that it is insufficient to judge models only by comparing $\mathrm{r}$-squared, we list for comparison the $\mathrm{r}$ squared values for the PG\&E WRF-format regressions (load as a function of WAVGDRY and WAVDRYSQ, as discussed above), and for the regressions of THISUM, THISUM1, and THISUM2 on SUMLOAD as formerly used by previous CEC forecasts. The PG\&E WRF-format regressions have nearly as high r-squared as do the stepwise regression models; this is particularly impressive considering that the stepwise regression models were developed by explicitly maximizing r-squared.

In general, fits are best for CEC region 2 and for PG\&E zone $S$, both of which represent the Sacramento area. For example, for CEC region 2 Summer, a model with just two explanatory variables, THISUM and CDDSUM90, explains 92 percent of the variance of load about the mean. The fits for CEC region 4 and PG\&E zone $X$ are considerably poorer than the fits for the other regions and zones. The poorer fits for CEC Region 4 and PG\&E Zone $\mathrm{X}$ are not surprising, since these two areas are relatively mild, and may be more climatically diverse than the other areas as well. In Chapter 7 we re-estimate daily load regression models for CEC region 4 , using an alternative set of weather data to construct explanatory variables.

\subsection{Assessing Model Fit}

While r-squared can provide a useful summary measure of a model's explanatory capability, it has several limitations, and does not describe all aspects of interest in assessing model fit. Among the chief limitations of r-squared are: (1) it does not necessarily represent the predictive capability of the model for the aspects of most interest; for example, the model may give biased estimates for peak load days, but $r-$ squared alone will not indicate this; and (2) r-squared describes model fit using the same data used to develop the model as to assess it; out-of-sample assessment of the model may provide a better indication of how well the model can work for forecasting purposes. 
For these reasons, it is valuable to develop intuitions regarding these findings by reviewing graphical summaries. Figure 3-1 is a time-series plot of model residuals (observed minus fitted values of daily average central cooling energy) for CEC regions 2, 3, and 4 over a period of five years. For each region, the residuals are the result of three separate regressions, one for each of Spring, Summer, and Fall seasons. The gaps in the plot are for the winter season (November through March) for which daily cooling loads are assumed to be zero.

The plots for CEC regions 3 and 4 show some striking patterns. In particular, the regression models for region 2 tend to overpredict loads in 1985 and 1986, and underpredict loads in 1988, with consistent overprediction for a substantial period in summer 1989. The models for region 4 tend to overpredict in 1986 and 1989, and underpredict in 1988 and in mid-summer 1985. We do not know how to explain the fairly major response shifts shown for region 3, or the extent to which the patterns in AMP sample load indicate patterns in regional load, but the effect may be important in assessing the value of forecasts. ${ }^{15}$

\footnotetext{
is Such annual shifts have implications for the time period over which models are developed. One possible treatment of this effect, for modeling purposes, is to express all loads as deviations from the annual means, as suggested by Kristov (Kristov 1991).
} 
Table 3-1. Weather Stations Assigned to PG\&E Zones and CEC Regions for Cooling End-Use Models Based on 1985-1989 AMP Data

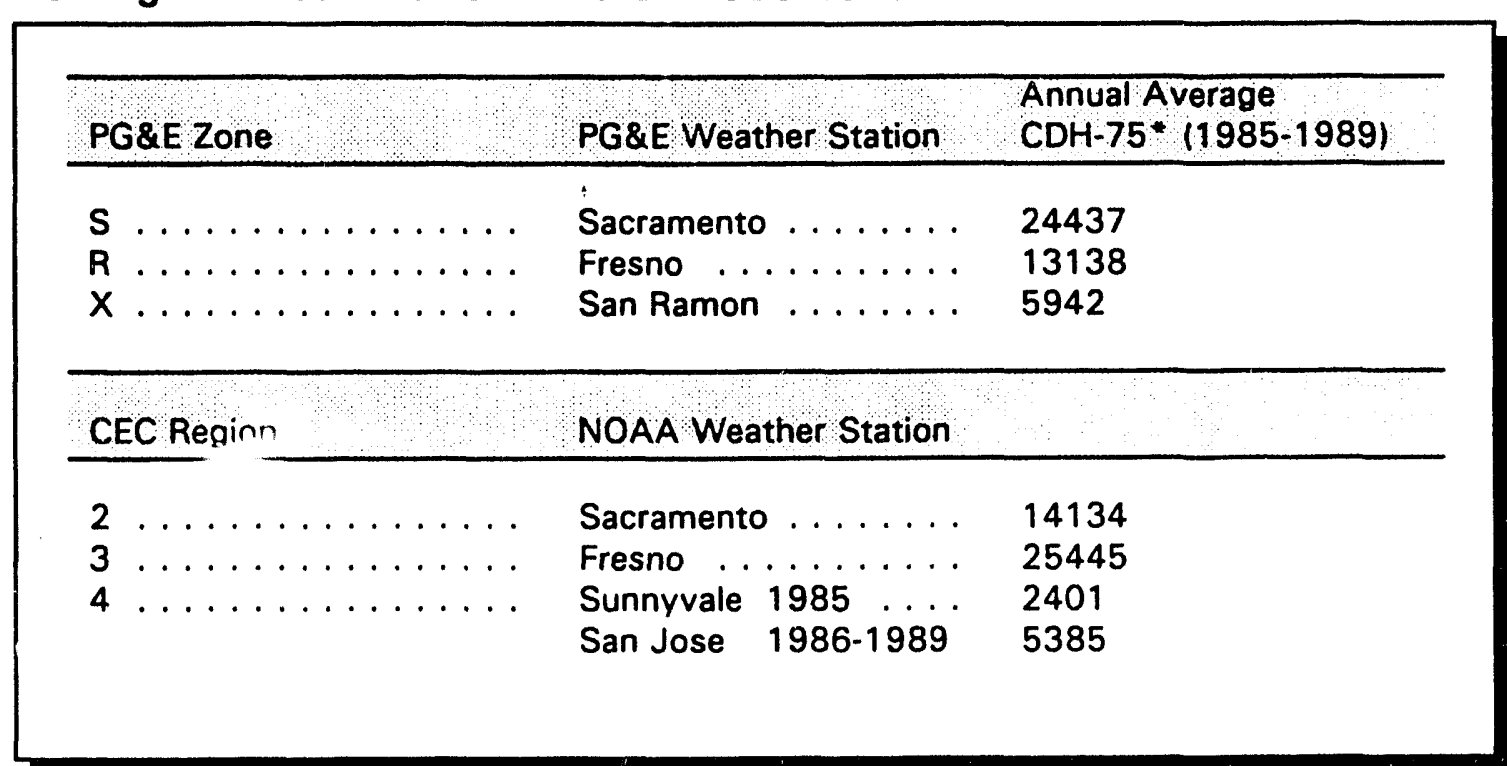

*Annual $C D H-75=\sum_{h=1}^{8760} \max (d r y$-bulbtemperature $[h]-75,0)$ 
Table 3-2. Variables Used in Daily Regressions

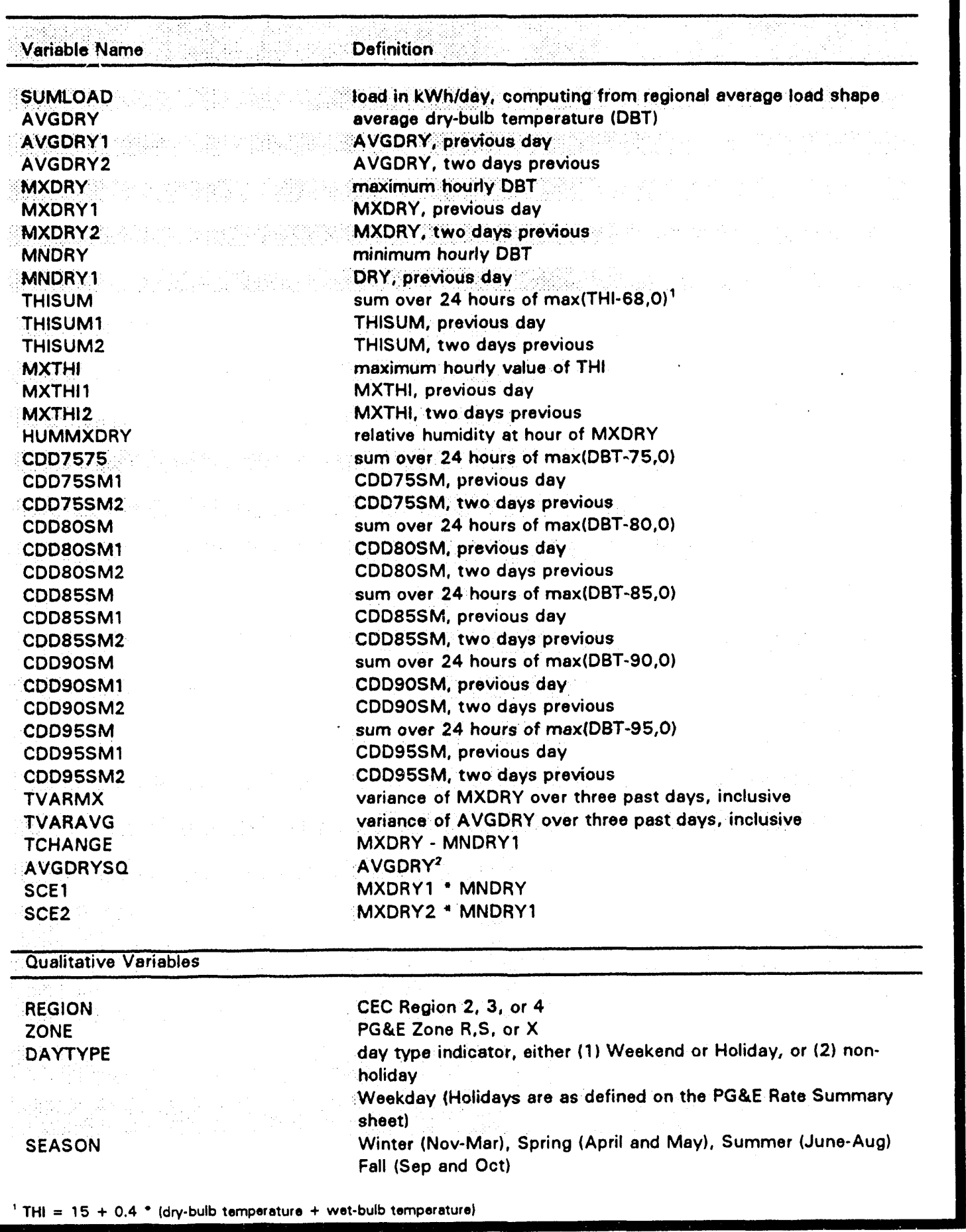


Table 3-3. Summary of Regression Results for CEC Regions 2,3,and $4^{1}$

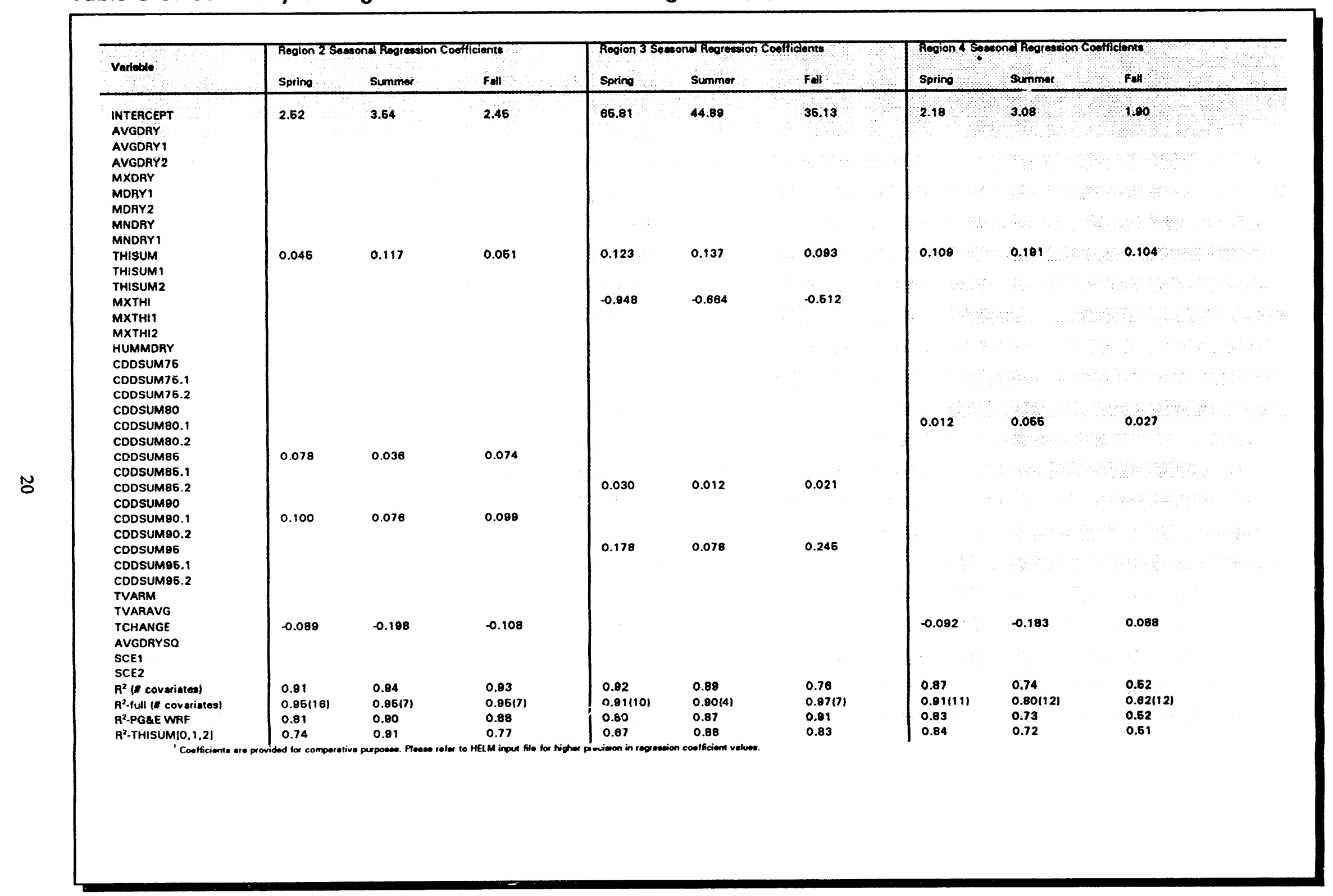


Table 3-4. Summary of Regression Results for PG\&E Zones ${ }^{1}$ S,R, and X

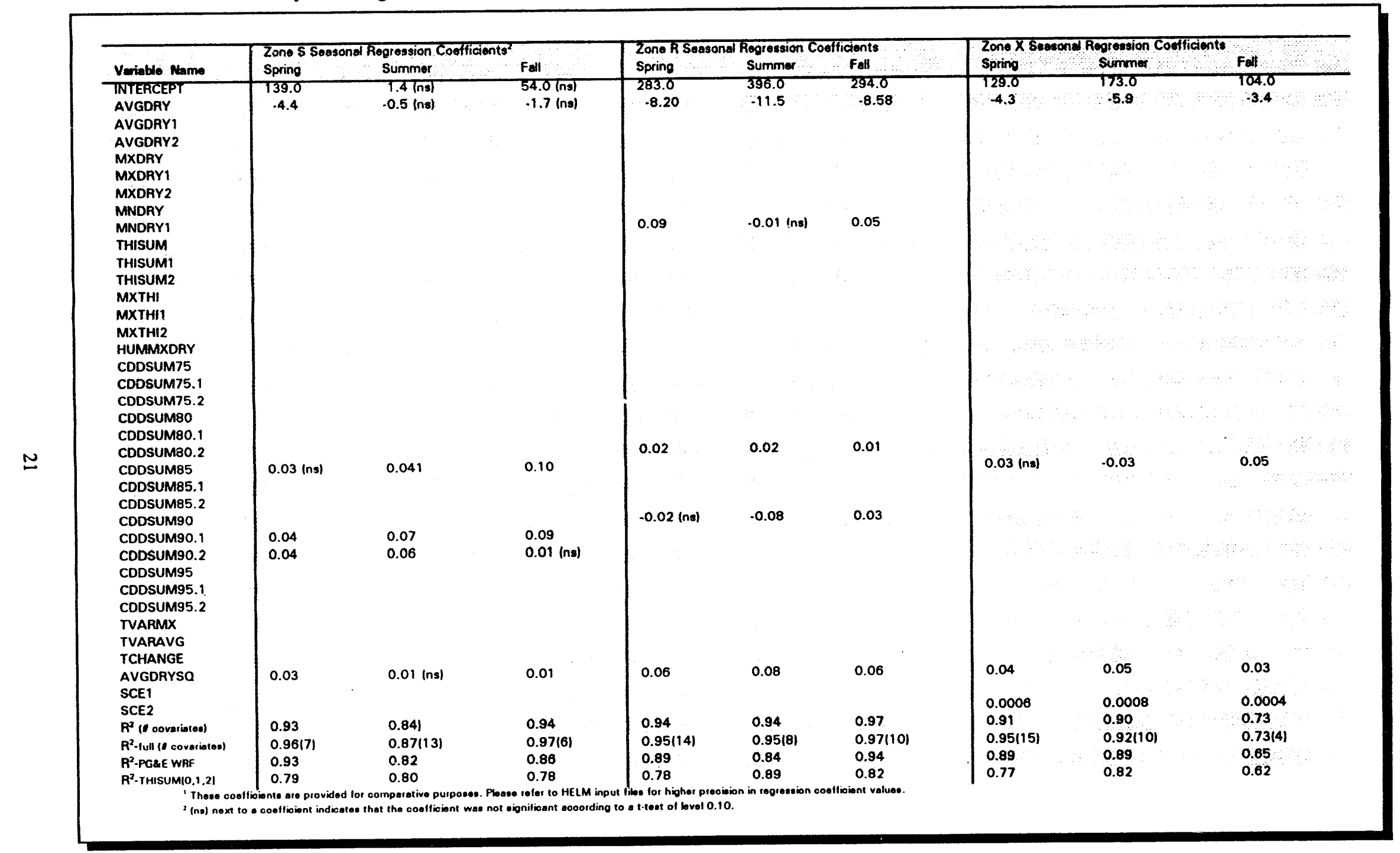


Region 2
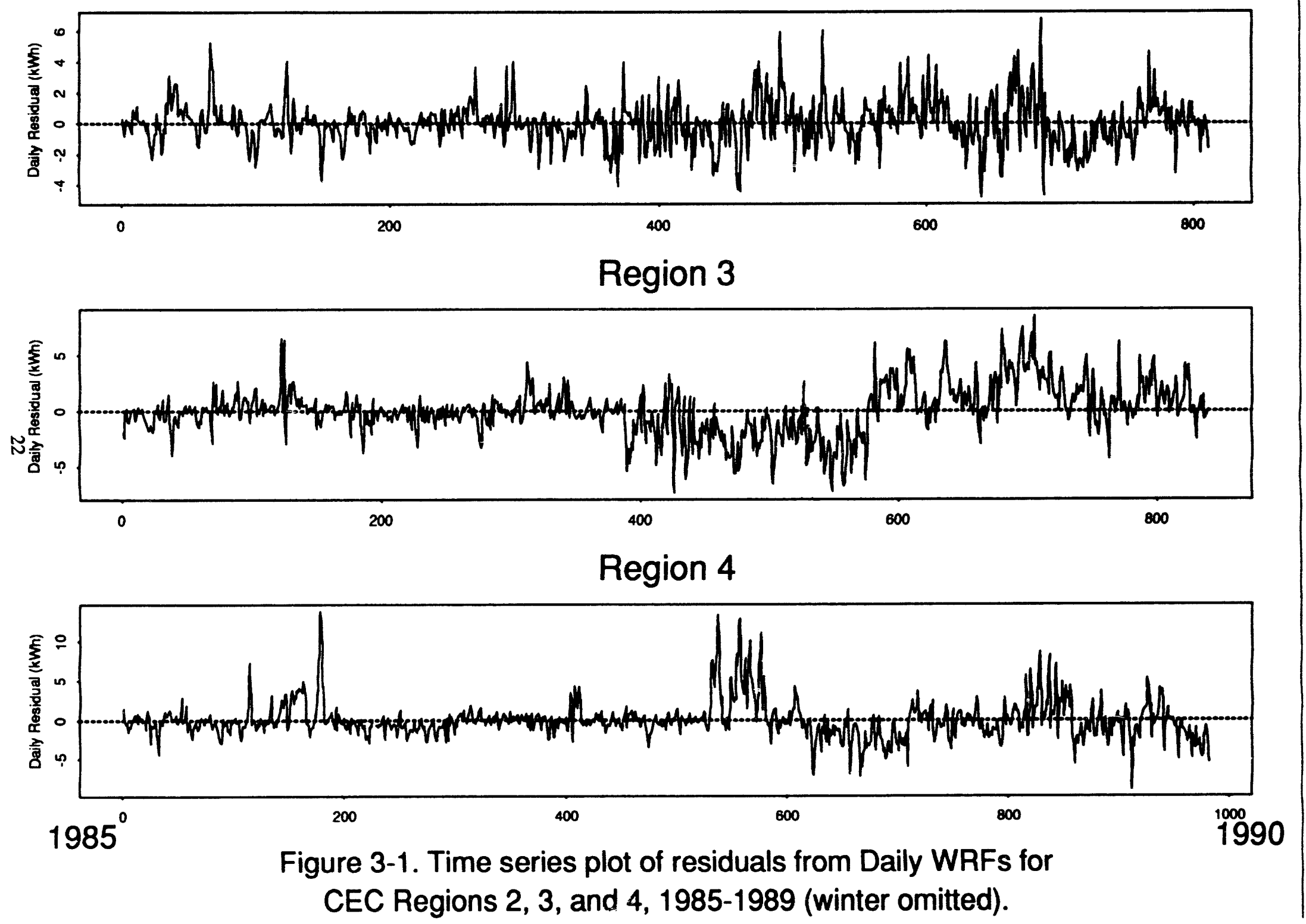


\section{Binned Hourly Energy Load Shapes}

In this chapter we describe how we developed specifications for HELM's allocation of daily central cooling energy estimates to the hours of the day. ${ }^{16}$ HELM performs the allocation by assigning each day to a fixed 24-hour load shape according to season, day of week, and weather measurements for that day. Below, we discuss how we derived libraries of fixed load shapes from the AMP sample data using bin definitions previously specified by PG\&E. We continue discussion of our work on binned load shape libraries in Chapter 7, where we assess the variability of the load shapes within a bin and also describe our initial investigation of possible alternative bin definitions.

\subsection{Approach}

The principle behind defining load shape bins is to use characteristics external to load data, such as weather and day of the week, to separate days into groups such that load shapes are similar within a group, relative to load shapes in other groups. Grouping, of course, will depend on the measure or measures of similarity used, which in turn should depend on which aspects of the load shape are of most importance in forecasting. All binning described in this chapter is based on load shape descriptions in which hourly load is given as a proportion of total daily load; we refer to these as load profiles or normalized load shapes. Load shapes for a bin are computed using HELM's algorithm, described below, which is based on the use of load duration curves.

PG\&E previously developed load shape bin definitions for zones $R, S$, and $X$ using only 1989 AMP data and PG\&E weather data. Bin definitions were based on three-day weighted daily average dry bulb temperature described in Chapter 3 . The bins were developed using HELM PC 1.0, and were based on data for an extended summer season, June through September. The bins were developed using a manual iterative procedure, in which bin temperature ranges were successively modified towards minimizing the sum of the hourly standard errors within a bin. Table 4-1 shows the adopted bin definitions.

\footnotetext{
${ }^{16}$ The daily energy estimates are computed according to daily Weather Response Functions, which are described in Chapter 3.
} 
LBL extended these definitions to a changed summer season definition (June through August) and to the Spring (April and May) and Fall (September and October) seasons in order to develop load shape for HELM input files. We used AVGDRY rather than WAVGDRY to make bin assignments. (See Table 3-2 for formal definitions of these quantities.) We also extended these definitions to the CEC Regions. We applied PG\&E zone $R$ bin definitions to CEC region 3, PG\&E zone $S$ bin definitions to region 2 , and $P G \& E$ zone $X$ definitions to CEC region 4. For cases in which the extended definition resulted in a bin with too few days being assigned, the bin was combined with the bin directly below it. This occurred in four cases for PG\&E zones and in two cases for CEC regions.

Data for all days assigned to a bin are used to compute a representative load shape for the bin. HELM's algorithm for computing this representative load shape is based on the use of a load duration curve. The procedure is described in detail in the HELM Manual (ICF Resources Inc. 1992). In brief, the algorithm is as follows: (1) determine the set of days falling into a bin, (2) normalize the loads in each day in the set by dividing each observed hourly load by the total daily load (optional), (3) compute an average load shape based by averaging the loads for each hour across the days in the set, (4) compute the load duration curve for each day by reordering the loads in each day from highest to lowest load, (5) compute the average load duration curve by averaging the hourly values of the daily load duration curves for all days in the set, and finally, (6) derive the load shape representation by reordering the loads in the load duration curve according to the relative magnitudes in the average load shape (e.g., the hour of the peak in the average load curve is assigned the magnitudes of the average peak hour in the load duration curve)

\subsection{Results}

The resulting load shape libraries are represented graphically in Appendix B. Figures B-1 through B-3 present the load shapes for PG\&E zone R for Spring, Summer, and Fall respectively. Figures B-4 through B-6 present PG\&E zone S; Figures B-7 through B-9 present PG\&E zone X; Figures B-10 through B-12 present CEC region 2; Figures B-13 through B-15 present CEC region 3; and Figures B-16 through B-18 present CEC region 4. Note that some load profiles in cooler bins (e.g., the load profile for the $66.2-75^{\circ}$ bin for Spring in PG\&E zone R) show small "bumps" in early morning hours. These bumps may correspond to ventilation loads for heating. ${ }^{17}$ The Region 4 load shapes for Fall and Spring (B-16 and B-18) are somewhat irregular, suggesting perhaps bins could be further

\footnotetext{
${ }^{17}$ It is our understanding that previously PG\&E smoothed these bumps away using a smoothing feature provided in HELM.
} 
collapsed, especially since the Summer load shapes for the region (B-17) vary little by temperature bin. However, the load shapes for the mildest of the PG\&E zones, X, are relatively smooth, and do vary considerable by temperature bin.

Table 4-1. Cooling Load Bins by PG\&E Zone for Spring, Summer and Fall Seasons"

\begin{tabular}{|c|c|c|}
\hline PG\&E Zone & \multicolumn{2}{|c|}{ Range of Daily Average Temperature (०F) } \\
\hline$\ldots \ldots$ & $\begin{array}{l}0.0-66.2 \\
66.2-75.0 \\
75.0-80.0 \\
80.0-85.0 \\
85.0-87.5^{1} \\
87.5-100.0\end{array}$ & $\begin{array}{l}0.0-66.2 \\
66.2-75.0 \\
75.0-80.0 \\
80.0-85.0^{2} \\
85.0-87.5 \\
87.5-100.0\end{array}$ \\
\hline$\ldots \ldots$ & $\begin{array}{l}0.0-62.1 \\
62.1-70.0 \\
70.0-75.0 \\
75.0-80.0 \\
80.0-85.0^{3} \\
85.0-100.0\end{array}$ & $\begin{array}{l}0.0-62.1 \\
62.1-70.0 \\
70.0-75.0 \\
75.0-80.0\end{array}$ \\
\hline$x$ & $\begin{array}{l}0.0-58.6 \\
58.6-67.5 \\
67.5-72.5 \\
72.5-77.5 \\
77.5-100.0\end{array}$ & $\begin{array}{l}0.0-58.6 \\
58.6-70.0\end{array}$ \\
\hline
\end{tabular}

- these bins were used to develop load shape representation libraries for HELM daily models

'except for Spring: range extended to $85-100$

2 except for Spring: range extended to 80-100; and Fall: range extended to $80-87.5$

${ }^{3}$ except for Spring: range extended to $80-100$ 
Table 4-2. Cooling Load Bins by CEC Region for Spring, Summer and Fall Seasons ${ }^{\bullet}$

\begin{tabular}{|c|c|c|}
\hline CEC Region & \multicolumn{2}{|c|}{ Range of Daily Average Temperature (OF) } \\
\hline$\ldots \ldots \ldots \ldots \ldots$ & $\begin{array}{l}0.0-66.2 \\
66.2-75.0 \\
75.0-80.0 \\
80.0-85.0 \\
85.0-87.5^{1} \\
87.5-100.0\end{array}$ & $\begin{array}{l}0.0-66.2 \\
66.2-75.0 \\
75.0-80.0 \\
80.0-85.0 \\
85.0-87.5 \\
87.5-100.0\end{array}$ \\
\hline 3 & $\begin{array}{l}0.0-62.1 \\
62.1-70.0 \\
70.0-75.0 \\
75.0-80.0 \\
80.0-85.0^{2} \\
85.0-100.0\end{array}$ & $\begin{array}{l}0.0-62.1 \\
62.1-70.0 \\
70.0-75.0 \\
75.0-80.0\end{array}$ \\
\hline$\ldots \ldots \ldots \ldots$ & $\begin{array}{l}0.0-58.6 \\
58.6-67.5 \\
67.5-72.5 \\
72.5-77.5 \\
77.5-100.0\end{array}$ & $\begin{array}{l}0.0-58.6 \\
58.6-70.0\end{array}$ \\
\hline
\end{tabular}

- these bins were used to develop load shape libraries for HELM daily models

1 except for Spring: range extended to 80.100

2 except for Spring: range extended to $80-100$ 


\section{Hourly Energy Models}

In this chapter, we examine models now available in HELM that directly allocate annual cooling energy to hours of the year. This procedure is an alternative to the two-stage allocation procedure, which has been used by PG\&E and CEC, and for which we developed models in Chapters 3 and 4. The models we examine are based on linear regressions developed using hourly weather data to model hourly average metered AMP central air conditioner loads. Our approach is to first consider a variety of model specifications using CEC region 3 Summer data. ${ }^{18}$ Based on these investigations, we then proceed to develop models for Summer season cooling end use for CEC regions 2 and 4. ${ }^{19}$ Formal model assessment issues are left to Chapter 6. Additional exploratory issues considered in the development of the models are also discussed in this Chapter.

\subsection{HELM Hourly Weather Response Functions}

The most recent version of HELM PC (Version 2.0) offers the capability to develop and use hourly Weather Response Functions as well as daily Weather Response Functions (WRFs). Hourly models allow for a more explicit connection between diurnal weather patterns and cooling load than do daily models, since daily models also require a separate procedure to allocate daily energy use to the hours of the day. ${ }^{20}$

The format HELM uses for the hourly WRFs parallels that used for the daily WRFs. HELM allows the user to model hourly load as a linear function of weather variables, where models may be specified separately according to user-defined seasons and daytypes. ${ }^{21}$ HELM interprets the hourly loads resulting from the WRF as a relative

\footnotetext{
${ }^{18}$ We selected CEC Region 3 for our exploratory analyses because it is the region (among the CEC Regions) with the highest average central air conditioner UEC.

${ }^{19}$ Hourly models were not developed initially for PG\&E Zones R,S, and X pending the outcome of the overall evaluation of this approach in Chapter 6.

${ }^{20}$ A time-series based approach to hourly load modeling is theoretically appealing: however, HELM does not directly provide for such an approach; we did not investigate the development of such models.

${ }^{21}$ The model for a given combination of season and daytype may be piecewise linear, consisting of several segments, with each segment defined according to the values of weather variables, with different parameter coefficients specified for each segment.
} 
percentage of total energy within a forecast period, which is typically a calendar year. These relative percentages are subsequently scaled to reflect an exogenously specified (from an annual energy forecasting model) total energy use for the period specified in the HELM Forecast file. For flexibility, we developed our models outside of the HELM framework using SAS, and subsequently reformatted the resulting model specifications as HELM input files.

\subsection{Weather and Load Data}

Table 5-1 lists the covariates considered for our hourly models. We based this list, in part, on the weather variables considered in the hourly models examined by Kristov (1991). ${ }^{22,23}$ Note that the variables wind speed (WNDSPD) and cloud cover (CLDCVR), which were not considered in the construction of our daily Weather Response Functions, are available only for NOAA stations and not for PG\&E weather stations. We include Temperature-Humidity Index (THI) in several forms: THI (for THI base 0), THI-base-68 (THIB, defined as the greater of THI minus 68 , or 0 ), as well as the six-hour lagged sums of THIB ${ }^{24}$ (THIBSUM6) and the squared value of THIB (THIBSQ). Some other possible transformations of the basic weather variables (e.g., different bases for THI) are discussed below.

As discussed in Chapter 3, some of the AMP central air conditioner load data includes small ventilation loads incurred during heating operation. Although the models examined in this chapter are based on Summer data only, this load data may also include such

\footnotetext{
2 Note that the models we discuss are developed on the basis of five years of data (1985-1989), whereas Kristov (1991) examines a similar set of models using one year of data (1989).

${ }^{23}$ In linear regression models for hourly average AMP loads, Kristov found that using all four primary weather variables (dry-bulb temperature, dew point, cloud cover, and wind speed) had considerably more explanatory power than THI alone, and that adding lagged THI information greatly improved the explanatory power of both the four-variable and the THI-alone regression models. Kristov considered two types of daily regressions, one modeling total daily load (SUMLOAD) and one modeling maximum hourly load (LOADMAX). In general, fits were better for LOADMAX than for LOADSUM, which has implications for modeling peak loads, in particular. Kristov found that models using the 24-hour sum of THI-base-68 (THISUM) for the present day and for the two previous days (as motivated by the CEC's current practice for their Peak Demand Forecast model), and similarly, models using the 24-hour sum of cooling degree day base 75 (CDDSUM) for the current day and the two previous days, provided the highest explanatory capability for the linear regression models, among the five models considered.

${ }^{24}$ Kristov also used a lag period of six. Based on our examination of the partial autocorrelation estimates of residuals from Model 1, six hours appeared to be an appropriate lag period (although the "best" lag period could vary depending on hour of the day).
} 
ventilation loads, particularly in the early morning or late evening hours. Load shape representations (Figure B-11, B-14 and B-17) indicate such occurrences are minor for Summer but they may be more important for modeling milder seasons and regions, for example some load shapes for Spring region 2 (Figure B-10) shows an 8 a.m. "bump".

\subsection{Exploratory Regression Results for CEC Region 3}

Using previous research and forecasting practices as a guide, we examined four linear regression models, in each case using only data for Summer (June through August) in CEC region $3 .^{25}$ The data for each hour were modeled separately. Tables 5-2 through 5-4 summarize results for the first three models, representing 72 separate regressions (24 hours for each of three sets of explanatory variables). The tables show model r-squared and note variables for which the regression coefficient was statistically significant at or below the 0.05 level.

\section{Model 1. Load = f (WETBLB, DRYBLB, CLDCVR, WNDSPD, error)}

This model uses the four basic NOAA weather measurements routinely available on hourly weather data tapes. Table 5-2 summarizes Model 1 results. For all hours, rsquared is considerably lower than those for the other models considered below. Model fit, in terms of $r$-squared, is best for the late afternoon and evening hours. For example, r-squared in each of the hourly models for 5 p.m. through 10 p.m. is above 0.75 . Examination of residuals reveals that part of the reason for the relatively poor fits is that in each model, load is considered linear in temperature throughout the range of observed temperatures, whereas in reality cooling load is near zero for most hours below a sufficiently cool threshold. That is, load increases in temperature only in the weather range in which cooling loads occur.

Except for the intercept, coefficients are positive (if they are significantly different than zero). The regression coefficient for cloud cover (CLDCVR) is significantly different than zero in all hours after 10 a.m. but is significantly different than zero in no hours before $10 \mathrm{a} . \mathrm{m}$. The regression coefficient for wind speed (WNDSPD) is significantly different than zero at the 0.05 level for only a few hours: 5 a.m., 8 a.m., 9 p.m., 10 p.m., and 11 p.m., with a positive coefficient for each of these hours. The addition of two variables WNDSPD and CLDCVR together explain less than 1 percent additional variance in load over a model with WETBLB and DRYBLB alone (that is, WNDSPD

\footnotetext{
${ }^{25}$ It may be more appropriate to use a regression procedure which accounts for the autocorrelation of the residuals, as did Kristov (1991). However, appropriate software was not available at the time of analysis.
} 
and CLDCVR together have a marginal r-squared of less than 0.01 relative to the WETBLB and DRYBLB model.)

Model 2. Load = f (THIB, THIBSUM6, error)

This model is motivated by the use of the weather index THI used by CEC's Peak Demand Forecasting Model (which uses THI both in allocation of annual energy to the daily energy and in allocation of daily energy to hourly energy). Table 5-3 summarizes Model 2 results.

THI, as a fixed function of wet-bulb and dry-bulb temperature, allows less flexibility in modeling than using wet-bulb and dry-bulb temperature independently. We found, as did Kristov (1991), that using THI alone as an explanatory variable resulted in considerably lower r-squared than those of Model 1. But by using THIB (THI -base-68, the maximum of THI minus 68 , or zero), we obtained higher values of r-squared than for Model 1 (results for the model using THIB only are not shown). In this case, the use of a "degree-day" concept successfully addressed the problem noted in discussing Model 1 of limiting model fit to temperature ranges with observed cooling loads.

Model 2 includes THIB, and in addition, THIBSUM6, which is the sum of THIB over the six hours preceding the hour modeled. The two THI-based variables of Model 2 together achieve higher values for r-squared than do the Model 1 variables: considerably higher for low-load morning hours and moderately higher for afternoon and evening hours (e.g., r-squared of 0.84 for 6 p.m. as opposed to the Model 1 r-squared of 0.81 ).

Model 3. Load $=\mathrm{f}(\mathrm{THIB}$, THIBSUM6, THIBSQ, DAYTYPE, error $)$

Model 3 incorporates two new variables to those of Model 2: a DAYTYPE variable (Weekday, or Weekend/Holiday), and the square of THI-base-68, THIB68SQ. Table 5-4 summarizes Model 3 results. These additions lead to considerably higher values of rsquared, particularly in the afternoon and evening hours, which is when the highest loads occur. For example, the r-squared values for 6 p.m., 7 p.m. and 8 p.m. are $0.87,0.88$, and 0.88 , respectively, for Model 3 , versus $0.84,0.84$, and 0.82 , respectively, for Model 2.

The coefficient for DAYTYPE is significantly different from zero (again at significance level at or below 0.05 ) only for 12 midnight and the hours between 1 p.m. and 5 p.m. inclusive, with a negative coefficient for Weekdays (lower loads) than on Weekends for 
these hours. ${ }^{26} \quad 27$ The lagged six-hour sum of THI-base-68, THIB68SLiM6, is significantly different than zero $(p<=0.0001)$ for all hour models.

Model 4. Load $=\mathrm{f}$ (WETBLB, DRYBLB70, CLDCVR, WNDSPD, THIBSQ, THIBSUM6, error),

We also examined a fourth model but do not summarize results in a table. This model represents Model 1 with modifications to address some of the deficiencies noted above: DRYBLB70 (the maximum of DRYBLB minus 70 , or zero) replaces DRYBLB, and second-order (THIBSQ) and lagged (THIBSUM6) weather information are added. Nevertheless, the r-squared for this model is only slightly better (by 0.01 or less) than those for Model 3.

Based on these results, Model 3 seems to represent the best model among those considered above, considering fit, parsimony, and the limitations of the weather data base. With respect to Model 4, which had a very slightly higher r-squared, two of the weather variables (CLDCVR and WNDSPD) are not available from the PG\&E weather stations, which would be needed to model hourly loads for the PG\&E zones. In addition, it is also likely that the choice of base temperature for DRYBULB70 would also change for other CEC regions and PG\&E zones.

\subsection{Regression Results for the Remaining CEC Regions}

We then estimated regression coefficients for CEC regions 2 and 4 using the structure of Model 3. Tables 5-5 and 5-6 summarize Model 3 fit for these CEC Regions (as noted above, Model 3 results for CEC region 3 are summarized in Table 5-4). Figure 5-1 presents the coefficients from the models developed for all three CEC regions graphically. This figure shows all coefficients for each hour, whether or not the coefficient was found to be statistically significant. The coefficients for each covariate show characteristic diurnal patterns across the regions, but in general interpretation of the coefficients is complicated by the fact that THIBSQ (covariate of the third column) is a function of THIB68 (covariate of the second column).

${ }^{26}$ Since DAYTYPE is a binary-valued variable, it has the same effect as allowing two different intercepts for the model (one for Weekday, and one for Weekend/Holiday).

${ }^{27}$ We also introduced, but ultimately did not include, day of the week as a variable in Model 3 . Between 2 p.m. and 6 p.m. there appears to be a significant contrast between mid-week and Saturday effects. However, this addition only explains about an additional 1 percent variance for each hour as compared to Model 3 . 
For each of the three CEC regions, model r-squared are fairly high from early afternoon to evening. R-squared for the late afternoon and early evening models (between 4 p.m. and 8 p.m.) is lowest for CEC region 4. For both CEC region 2 and CEC region 4, model $r$-squared is remarkably low for some hours of the day: less than 0.50 and as low as 0.24 between $4 \mathrm{a} . \mathrm{m}$. and $10 \mathrm{a} . \mathrm{m}$. for CEC region 2, and less than 0.50 between 6 a.m. and 9 a.m. for CEC region 4. These low r-squared values are in part a consequence of the low occurrence of cooling loads in these hours and perhaps heating ventilation loads. Given the small proportion of cooling loads which occur during these early morning hours, the low r-squared values are probably of little concern.

In Chapter 6, the results of our evaluation of these models is presented.

\subsection{Additional Thoughts on Hourly Model Development}

The use of hourly weather response functions is a relatively new application for HELM. We have taken this opportunity to consider an alternative approach and issues that stem from the basic analysis presented above.

\subsubsection{Pooled-Hours Models vs. Hours-Separate Models}

As an alternative to the hours-separate models presented above, we also pooled all hours and estimated coefficients for each of the four models, using Summer data for CEC region 3 . In this regression, we allowed the intercept term to vary depending on hour of the day, but otherwise the data were taken without regard to hour (that is, an analysis of covariance model with first-order effects of the categorical variable HOUR.) This pooledhours model restricts the (multidimensional) regression plane to be the same for all hours, except for a shift in location of the plane corresponding to the coefficient for hour (an intercept term). ${ }^{28}$ In contrast, among a set of hours-separate models the regression plane can vary freely. Table 5-7 summarizes these models. Although the r-squared for these models is high ( 0.92 for example, for Model 3 with hour as a categorical variable) the models do not predict loads as well as do the hours-separate models.

Defining prediction error as the sum of squared residuals for a given set of hours, we compared the hours-pooled to the hours-separate models. Prediction error for the hourspooled model is, of course, necessarily higher than for the corresponding hourly

${ }^{28}$ In a simple linear regression (one covariate) the slope of the regression line would remain the same but shift in position along the direction of the $y$-axis. 
regression model. For example, the prediction error for 7 p.m. is 38.4 for the hourspecific Model 3, but 41.5 (or 8 percent higher) for the hours-pooled Model 3.

These findings underscore the problem of relying too heavily on r-squared as the sole basis of judging models. ${ }^{29}$ In this case, a series of individual hourly models, each with lower r-squared, has better predictive capabilities than a single, pooled model with higher r-squared. This observation, in turn, motivates our examination of the comparative predictive capabilities of all of the models in Chapter 6.

\subsubsection{Long-Term Temporal Patterns in Model Residuals}

In our examination of residuals from the annual regression models (Chapter 3), we noted some strong temporal patterns. These patterns were also observed in our analysis of hourly loads.

Figure 5-2 shows the annual distribution of residuals from Model 3 for 7 p.m. load; the solid horizontal line at zero indicates the average residual. The distribution of residuals varies dramatically from year to year: the third quartile of the distribution of residuals in 1986 is at about zero, which is the same level of the first quartile of the distribution of residuals in 1988: $75 \%$ of the 19867 p.m. loads are over-predicted by the hourly model, whereas $75 \%$ of the 19887 p.m. loads are underpredicted by the hourly model. Figure 5-2 also shows the time-ordered values of the 7 p.m. residuals together with a smoothed line through the data. Residual patterns for other hours are similar. Note that Summer 1988 was particularly hot, which may motivate behavioral factors or correlate with other factors not adequately captured by our regression model. But when we examined patterns of Model 3 residuals for CEC Regions 2 and 4, we did not find similar patterns. Of course, strong residual patterns could also be a reflection of data anomalies, rather than of shifts in regional loads.

\footnotetext{
${ }^{29}$ For example, in a simple linear model: if the true model is Ey [i] $=a+b(x[i]-x)$, with $\operatorname{Var}(y)=\sigma^{2}: r^{2}$ increases as $r^{2}$ decreases, $b$ increases, and $\left.\sum_{i}(x[i]-x)\right)^{2} / n$ increases.
} 
Table 5-1. Variables Used in Hourly Central Air Conditioner Load Regressions

Variable Name

Definition

HOUR $\ldots \ldots$ Hour of day (1 through 24)

LOAD ... . . . . Regional average load for HOUR

AVGDRY ..... Average daily dry bulb temperature for day

CLDCVR' . . . . Amount of sky covered by cloud layers (0-10)

DAYTYPE ..... Weekday or Weekend/Holiday

DRYSUM6 ... $\sum_{\mathrm{h} \text { HOUR }-6}^{\text {HOUR-1 }}$ DRYTMP[h]

DRYTMP .... D Dry-bulb temperature

THI ....... Temperature-Humidity Index $[15+0.4 \times$ (DRYTMP + WETTMP)]

THIB ....... Max (THI-68, 0)

THIBSO ..... THIB * THIB

THIBSUM6 ... $\sum_{b \text {-HOUR }-6}^{\text {HOUR }-1}$ THIB[h]

WETTMP .... Wet-bulb temperature

WNDSPD' . . . . Wind speed in knots

${ }^{1}$ Not available from PG\&E weather stations 
Table 5-2. Summary of Model 1* Hourly Regressions for CEC Region 3 Summer Season

\begin{tabular}{|c|c|c|c|c|c|}
\hline \multirow[b]{2}{*}{ Hour of Day } & \multicolumn{4}{|c|}{ Significant Variables $(p \leq 0.05)$} & \multirow[b]{2}{*}{ r-squared } \\
\hline & WETBLB & $\begin{array}{l}\text { DRYBL } \\
\text { B }\end{array}$ & CLDCVR & WNDSPD & \\
\hline 1 & & & & & $\mathrm{n} / \mathrm{a}$ \\
\hline 2 & & & & & $n / a$ \\
\hline 3 & $\sqrt{ }$ & $\sqrt{ }$ & & & 0.59 \\
\hline 4 & $\sqrt{ }$ & $\sqrt{ }$ & & & 0.55 \\
\hline 5 & $\sqrt{ }$ & $\sqrt{ }$ & & $\sqrt{ }$ & 0.50 \\
\hline 6 & $\sqrt{ }$ & $\sqrt{ }$ & & & 0.46 \\
\hline 7 & $\sqrt{ }$ & $\sqrt{ }$ & & & 0.42 \\
\hline 8 & $\sqrt{ }$ & $\sqrt{ }$ & & $\sqrt{ }$ & 0.47 \\
\hline 9 & $\sqrt{ }$ & $\sqrt{ }$ & & $\sqrt{ }$ & 0.51 \\
\hline 10 & $\sqrt{ }$ & $\sqrt{ }$ & & & 0.50 \\
\hline 11 & $\sqrt{ }$ & $\sqrt{ }$ & $\sqrt{ }$ & & 0.54 \\
\hline 12 & $\sqrt{ }$ & $\sqrt{ }$ & $\sqrt{ }$ & & 0.58 \\
\hline 13 & $\sqrt{ }$ & $\sqrt{ }$ & $\sqrt{ }$ & & 0.63 \\
\hline 14 & $\sqrt{ }$ & $\sqrt{ }$ & $\sqrt{ }$ & & 0.69 \\
\hline 15 & $\sqrt{ }$ & $\sqrt{ }$ & $\sqrt{ }$ & & 0.72 \\
\hline 16 & $\sqrt{ }$ & $\sqrt{ }$ & $\sqrt{ }$ & & 0.75 \\
\hline 17 & $\sqrt{ }$ & $\sqrt{ }$ & $\sqrt{ }$ & & 0.77 \\
\hline 18 & $\sqrt{ }$ & $\sqrt{ }$ & $\sqrt{ }$ & & 0.81 \\
\hline 19 & $\sqrt{ }$ & $\sqrt{ }$ & $\sqrt{ }$ & & 0.82 \\
\hline 20 & $\sqrt{ }$ & $\sqrt{ }$ & $\sqrt{ }$ & $\sqrt{ }$ & 0.81 \\
\hline 21 & $\sqrt{ }$ & $\sqrt{ }$ & $\sqrt{ }$ & $\sqrt{ }$ & 0.81 \\
\hline 22 & $\sqrt{ }$ & $\sqrt{ }$ & $\sqrt{ }$ & $\sqrt{ }$ & 0.77 \\
\hline 23 & $\sqrt{ }$ & $\sqrt{ }$ & $\sqrt{ }$ & $\sqrt{ }$ & 0.73 \\
\hline 24 & $\sqrt{ }$ & $\sqrt{ }$ & $\sqrt{ }$ & & 0.68 \\
\hline
\end{tabular}




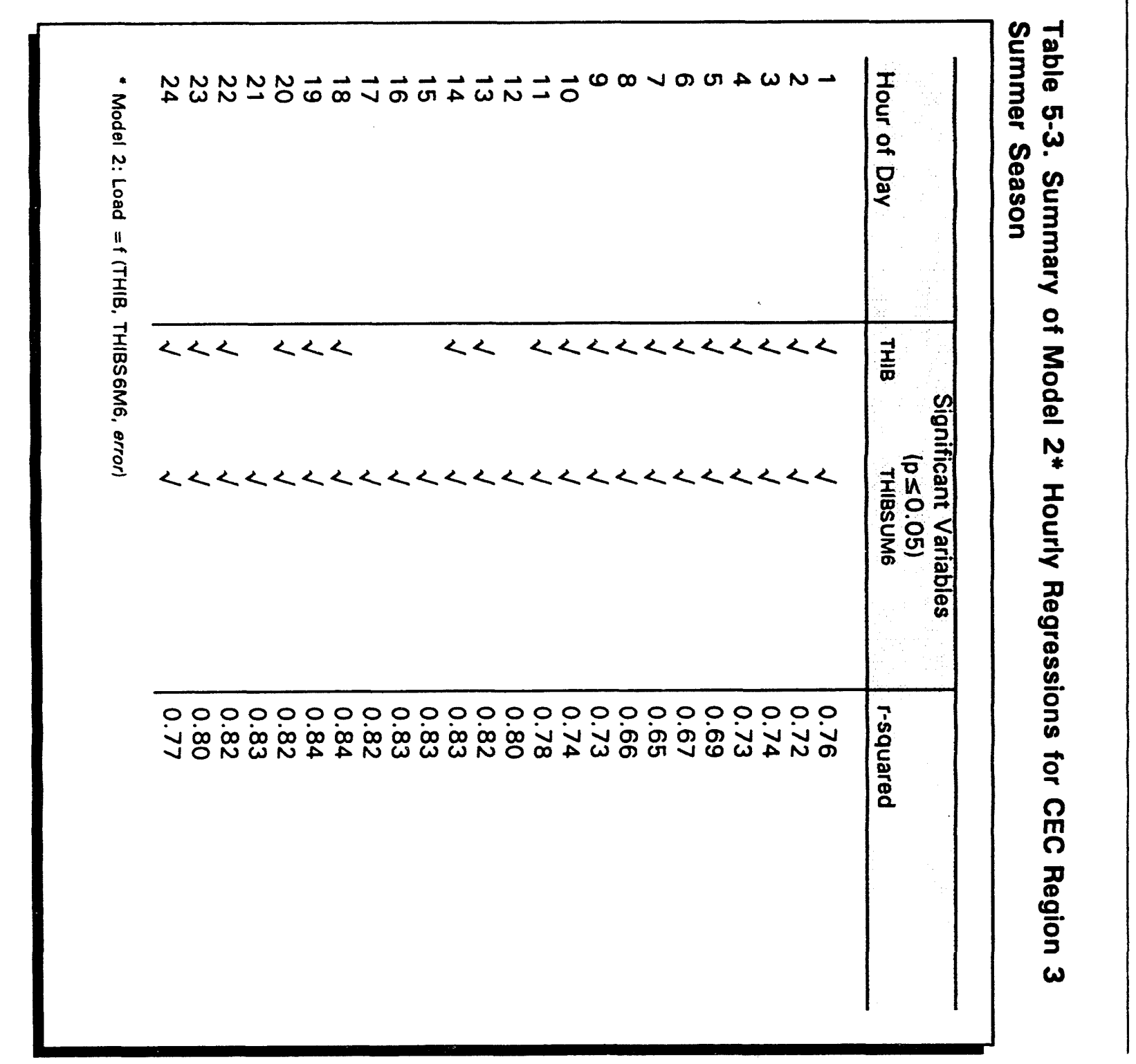




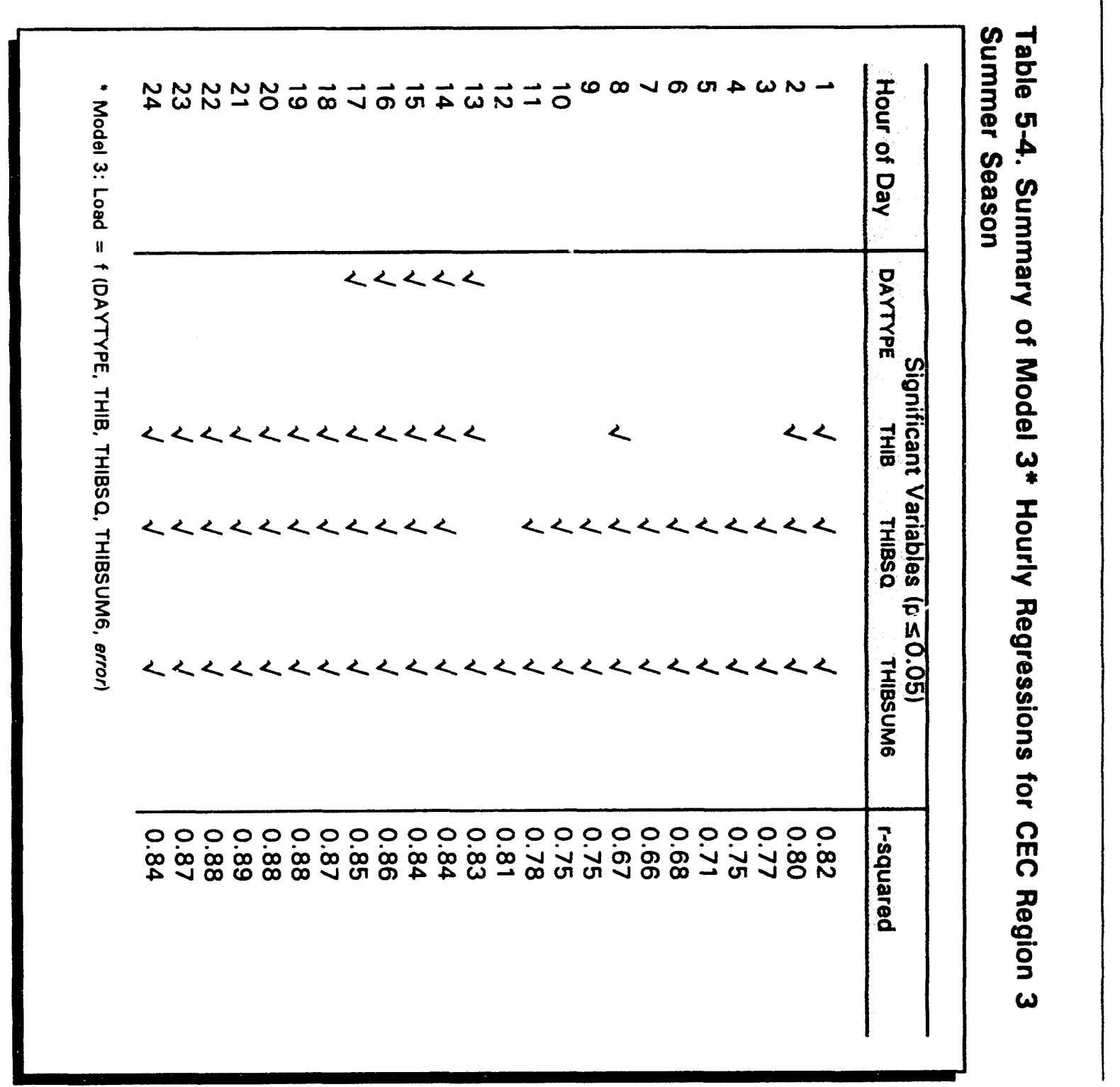




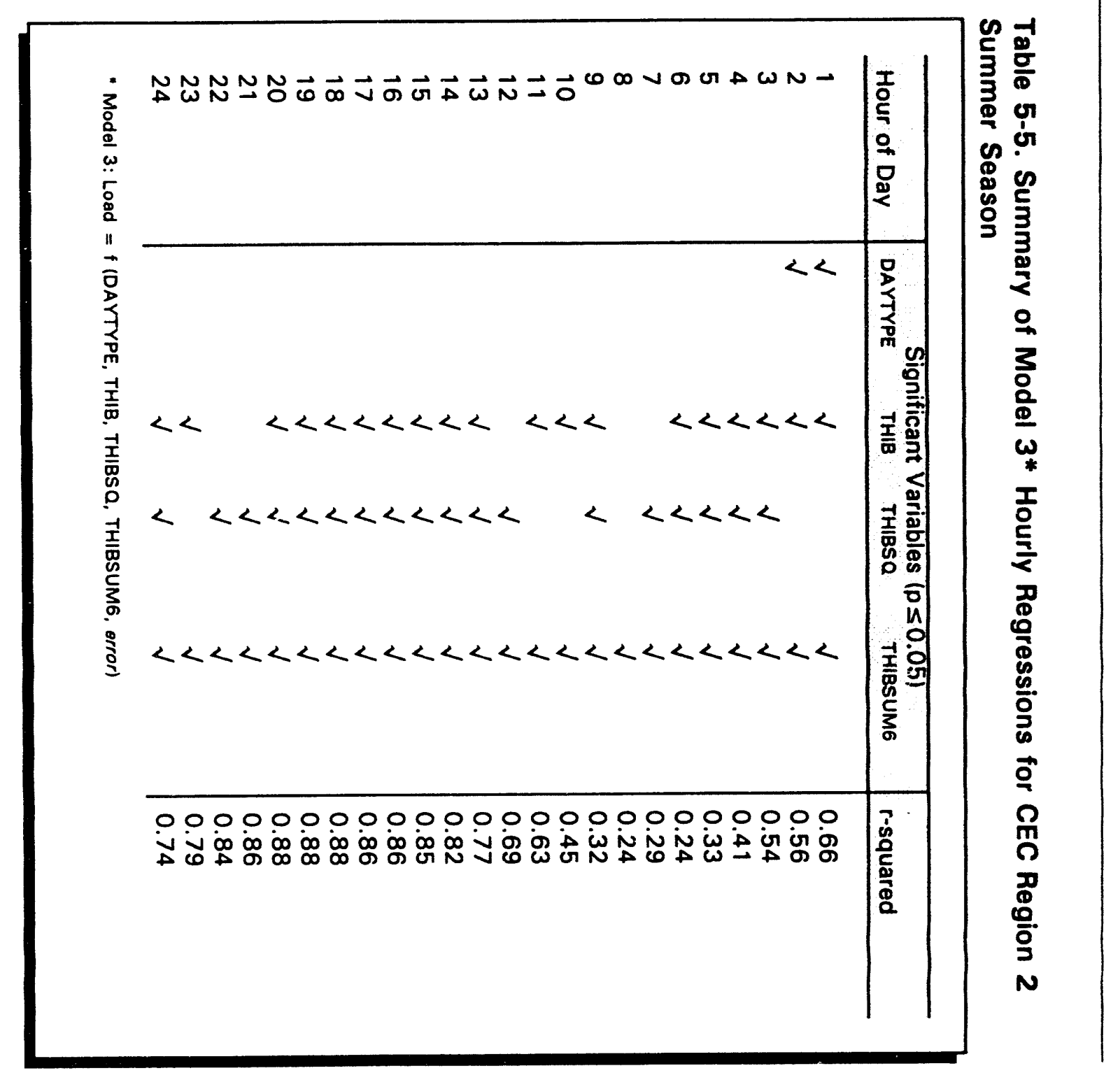




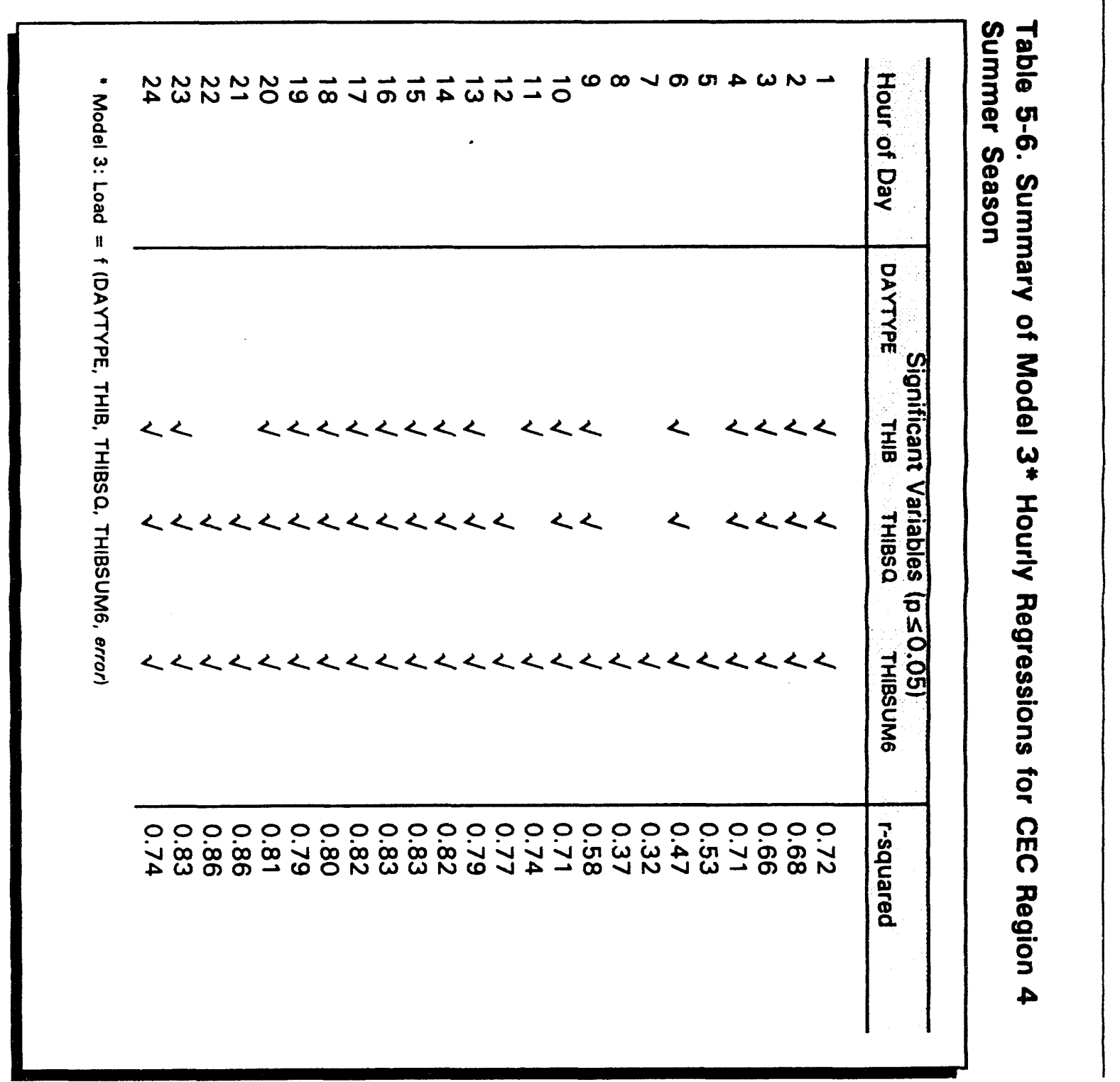


Table 5-7. Summary of Hours-pooled Regression Results for Summer CEC Region 3

Significant Variables

r-squared

HOUR*, DRYTMP,

Model 1.

CLDCVR, WNDSPD

0.77

Model 2.

HOUR*, THIB,

0.85

THIBSUM6

HOUR*, DAYTYPE,

Model 3.

THIB, THIBSO,

0.92

THIBSUM6

HOUR, WETTMP,

CLDCVR,WNDSPD,

Model 4.

THIBSUM6, THIBSO

0.92

" HOUR is a categorical ("factor") variable in these models. 
Table 5-8. Comparison of Prediction Errors in Selected Hours for Model 3 Based on Peak Day Subset to Model 3 Based on All Summer Days, for CEC Region 3

\begin{tabular}{|c|c|c|c|c|}
\hline \multirow{2}{*}{$\begin{array}{l}\text { Regression Model: } \\
\text { Load = f(THIB,THIBSQ,THIBSUM6, error) }\end{array}$} & \multicolumn{4}{|c|}{$\begin{array}{c}\text { Prediction Error Among Peak Day } \\
\text { Subset } \\
\text { Hour of Day }\end{array}$} \\
\hline & 16 & 17 & 18 & 19 \\
\hline $\begin{array}{l}\text { Hourly models based on all Surnmer days }{ }^{2} \\
\text { Hourly models based on peak day subset } \\
\text { (r-squared) }\end{array}$ & $\begin{array}{l}5.3 \\
3.6 \\
(0.60)\end{array}$ & $\begin{array}{l}5.3 \\
3.4 \\
(0.61)\end{array}$ & $\begin{array}{l}5.4 \\
2.5 \\
(0.64)\end{array}$ & $\begin{array}{l}5.5 \\
2.4 \\
(0.69)\end{array}$ \\
\hline \multicolumn{5}{|c|}{$\begin{array}{l}\text { Peak Day Subset is }=\text { days with } 50=\text { highest }=\text { values of Daily Energy/Annual Energy, } 1985- \\
1989, \text { based on PG\&E system load data provided by CEC } \\
2 \text { see Table 5-4 for summary of regression results for these models }\end{array}$} \\
\hline
\end{tabular}



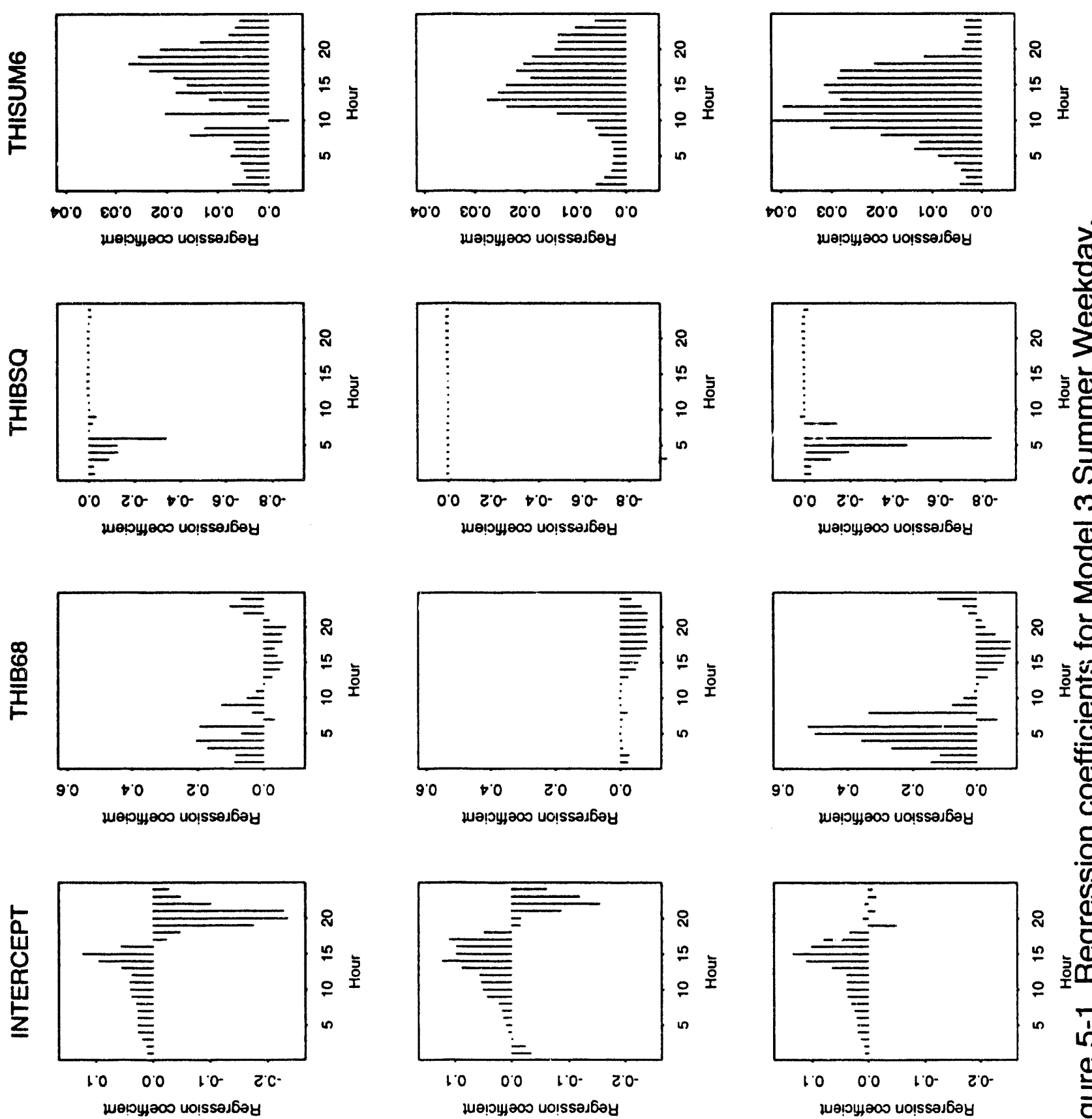

完

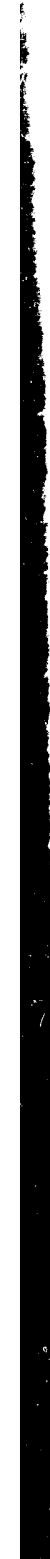


Annual distribution of residual from 7 p.m. hourly model

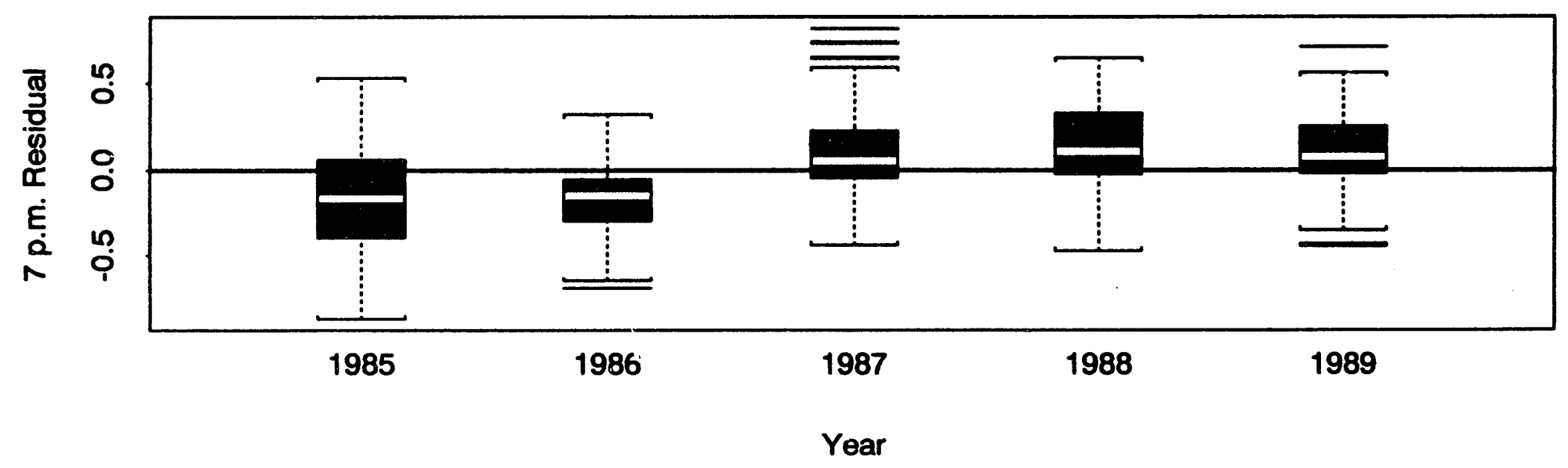

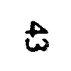

Residual from 7 p.m. hourly model vs. Time

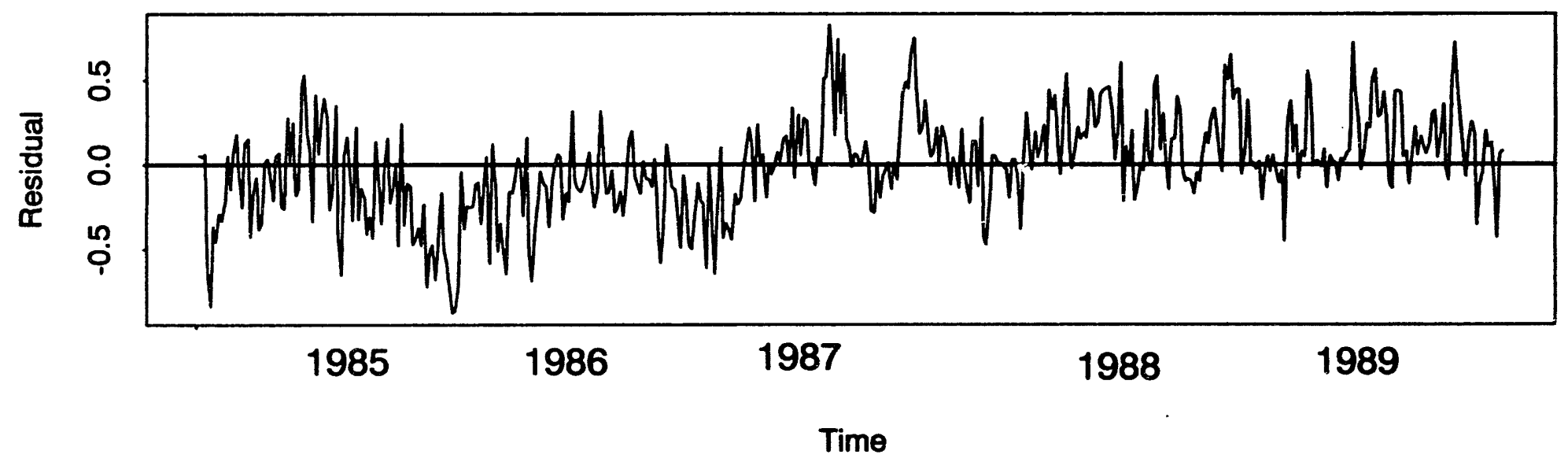

Figure 5-2. Temporal Patterns in Residuals for 7 p.m. Hourly Load Model. 


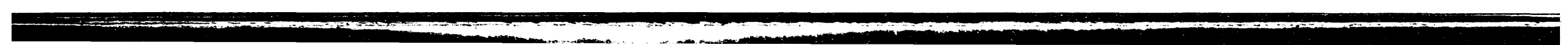




\section{Comparison of Cooling End Use Models}

In this chapter, we compare load shape predictions from a variety of models for the cooling end use. From the present report, we compare predictions from: (1) the daily WRFs along with the corresponding binned load shapes, discussed in Chapters 3 and 4; and (2) the hourly WRFs, discussed in Chapter 5. From previous LBL work (Eto and Moezzi 1992), we also compare (3) CEC's Peak Demand Forecasting Model using the grand time-temperature matrix developed by LBL from 1985-1989 AMP data; and (4) CEC's Peak Demand Forecasting Model using region-specific time-temperature matrices developed by LBL from 1985-1989 AMP data. Finally, from PG\&E's and CEC's existing peak models, we compare (5) PG\&E's daily WRFs used with corresponding PG\&E-developed binned load shapes; and (6) CEC's Peak Demand Forecasting Model using CEC's time-temperature matrix.

The comparisons are conducted by examining the differences between the sample average loads developed from the AMP data and the loads predicted by each model. Separate evaluations are conducted for each geographic forecasting aggregation (one for each of the three PG\&E zones and one for each of the three CEC regions). Note that not all models were developed for each PG\&E zone or CEC region.

This chapter is organized in six sections. First, we discuss model-specitic implementation issues that provide additional information on the methods used to develop model predictions for comparison to the AMP sample loads. Second, we describe the overall approach used to compare the models, including a discussion of the measures we developed to assess the performance of the models. In the third section, we present our findings for the models developed to forecast load shapes for CEC regions, while in the fourth section we present findings for the models developed to forecast load shapes for PG\&E zones. Fifth, we identify considerations that influence the interpretation of results. Sixth, we summarize our findings. 


\subsection{Computation of Predicted Loads}

In this section, we describe how we computed the prediction ${ }^{30}$ used for model comparison. Tables 6-1 and 6-2 indicate the models being compared for CEC regions and PG\&E zones, respectively. Note that not all models were developed or available for each CEC region and PG\&E zone. Also recall that, for the models reported on in Chapters 3 and 4, separate seasonal models were estimated for each CEC region and PG\&E zone.

\subsubsection{LBL Daily Regressions with LBL Load Shape Libraries (LBLWRF)}

Using the models developed in Chapter 3, we computed daily energy estimates for each CEC region (models summarized in Table 3-3) and for each PG\&E zone (models summarized in Table 3-4). We computed hourly load estimates by scaling the appropriate binned load shape (defined for each CEC region and PG\&E zone by Season, Daytype, and AVGDRY) by the daily energy estimate from the regression model. We refer to this model as LBLWRF. Recall that some days were not used in the regressions: (1) days in winter (November through March) season; and (2) days with average dry bulb temperature below the minimum specified for the regression (62.1 for CEC region 2 and PG\&E zone S, 66.2 for CEC region 3 and PG\&E zone R, and 58.6 for CEC region 4 and PG\&E zone $X$ ). Predicted cooling load for these days is always zero, following PG\&E convention. The five-year sum (omitting winters) of predicted loads for this model will therefore be less than for the AMP sample to which it is compared, because days below a certain temperature are modeled as zero but in fact the AMP sample may report small non-zero loads on these days ${ }^{31}$.

${ }^{30}$ Throughout this chapter, we use the term prediction loosely to indicate loads estimated by model on the basis of observed data, although they are technically not predictions since the same data is used to develop the models as to evaluate them. A more accurate term for such estimates might be backcasts.

${ }^{31}$ For accounting purposes, one could model the omitted days with a season-specific mean for those computed days. Under such a procedure, sample and model total energy would match (ignoring winter). 


\subsubsection{LBL Hourly Weather Response Function (LBLHWRF)}

Using the hourly WRFs of Model 3 (reported on in Chapter 5), we computed a second set of hourly load estimates. Model 3 consists of 24 separate hourly models for each CEC region for each of three seasons (spring, Summer, and fall, using the same season definitions given in Chapter 3) ${ }^{32}$ We derived daily load estimates for this model by adding together the 24 hourly estimates for each day. We refer to this model as LBLHWRF. The five-year sum (omitting winters) of predicted loads for this model is necessarily the same as that of the AMP sample.

These models were developed for CEC regions only, not for PG\&E zones, pending the outcome of the evaluation presented in this chapter.

\subsubsection{CEC Peak Demand Forecasting Approach with LBL Matrix (LBLMAT)}

LBL has previously developed space conditioning models for an older forecasting model used by CEC, called the CEC Peak Demand Forecasting Model (Eto and Moezzi 1992). This approach is similar to that of LBLWRF. First, an allocation of energy is made from year to day. Second, an allocation is made form day to hour. In this approach, a timetemperature matrix replaces the use of binned load shapes for the allocation of daily energy to hours of the day.

To generate daily hourly load shapes, we used a time-temperature matrix developed by LBL from AMP data. This matrix was developed using 1985-1989 AMP data from all regions to develop a "raw" matrix, and subsequently smoothing the surface of the resulting matrix. The development of this matrix has been described previously (Eto and Moezzi 1992). The matrix is used to generate a load shape for a day by assigning 24 values from the matrix, corresponding to 24 hourly values of THI. The resulting load shape is converted from the units in which the matrix is expressed ( $\mathrm{kWh} / \mathrm{hour})$ into a load profile by dividing by the sum of the 24 hourly loads, so that the load for each hour is expressed as a percentage of the total daily load.

This load profile is then re-scaled by multiplying each hourly value (normalized to sum to one) by a daily energy estimate computed in a separate phase of the model. LBL did not examine the methods used in this second phase of the CEC model. Accordingly, we rely on CEC's current specification of the daily energy allocation in its Peak Demand Forecasting Model and used them to develop predictions for CEC regions.

${ }^{32}$ Chapter 5 included summaries of results only for the summer season models. 
The CEC Peak Demand Forecasting Model for the residential sector allocates estimates of annual conditioning energy consumption to daily consumption according to the relative values of WTHI-DD for the day and long-term average annual THI-DD (see Table 3-2 for definitions of these variables). For a given day $i$, the allocation is as follows:

Daily Energy[i] $=($ WTHISUM[i]/ATHISUM) $* \mathrm{AC}$

where

WTHISUM $[i]=0.6 *$ THISUM $[i]+0.3 *$ THISUM $[i-1]+0.1 *$ THISUM $[i-2]$

THISUM

ATHISUM $\quad=\quad$ Long-term annual average sum of THISUM for the year

$\mathrm{AC}$

$=\quad \sum_{h=1}^{24} \max (T H I[h]-68,0)$

$=\quad$ Annual electricity consumption for central air conditioner $[\mathrm{kWh} / \mathrm{y}]$

For our model evaluations, we modified the procedure by (1) replacing ATHISUM by the sum of daily WTHISUM between 1985 and 1989; and (2) defining AC as the fiveyear (1985-1989) UEC for central air conditioner load, computed from the AMP sample. Thus, for day $i$ we scale the load profile generated from the time-temperature matrix by the factor:

$$
\text { Daily Energy[i] }=\left[\text { WTHISUM[i] } / \sum_{j \in D a y s(85-89)} \text { THISUMU]] } * \sum_{y e(85-89)} U E C[y]\right.
$$

Once again, these factors are defined separately for each CEC region (e.g. using data from the NOAA Fresno weather station and the UEC for CEC region 3 AMP sample to derive estimates for $\mathrm{CEC}$ region 3 ).

The modified computations necessarily produce a UEC which matches sample UEC for the five years combined ${ }^{33}$. Note that this energy allocation procedure is not directly based on modeling sample loads, whereas both LBLWRF and LBLHWRF are based the

\footnotetext{
${ }^{33}$ We considered re-scaling separately for each year, rather than using a five-year total, but rejected this since for appropriate comparison, regression model results would also have to be re-scaled to produce one-year UECs (which is unreasonable).
} 
AMP sample data ${ }^{34}$. This model produces daily energy estimates that, when summed, equal the corresponding five-year observed energy totals from the AMP sample (including winter). According to this model, the peak load occurs on the day with the highest value of WTHISUM.

\subsubsection{CEC Peak Demand Forecasting Model with LBL Raw region-Specific Matrices (LBLRAW)}

Using different time-temperature matrices, but the same daily energy allocation procedure as that described for LBLMAT, we derived another set of hourly and daily load predictions for CEC regions 2, 3, and 4. For each region, we used a time-temperature matrix based on data from that region alone, e.g. to model CEC region 2 we used a matrix which was derived on the basis of 1985-1989 AMP data for residences assigned to CEC region 2. We used "raw" (unsmoothed) matrices for these computations, whereas for the LBLMAT predictions we used a smoothed matrix.

These models were developed for CEC regions only, not for PG\&E zones.

\subsubsection{CEC Peak Demand Forecasting Model with CEC Matrix (CECMAT)}

Using the time-temperature matrix currently used by CEC (not developed by LBL), along with the same daily energy allocation procedure described previously for LBLMAT (also developed by CEC), we derived CECMAT predictions. Once again, these models were developed for CEC regions but not PG\&E zones.

\subsubsection{PG\&E Daily Regressions with PG\&E Load Shape Libraries (PGEWRF)}

We generated hourly load predictions for PG\&E zones for 1985-1989 from PG\&E's current HELM input files of daily WRFs and corresponding binned load shapes (which were developed by PG\&E on the basis of 1989 AMP data). ${ }^{35}$ We re-scaled the energy estimates (which were expressed in units of PG\&E sector-wide energy) to produce the

\footnotetext{
${ }^{34}$ One might also consider using the load shape derived from the time-temperature matrix itself, rather than the current practice of normalizing it and re-scaling it with this "external" allocation procedure.

${ }^{35}$ Estimates were received by LBL from CEC 3/26/92 (with letter dated 1/24/92) in the files CZRRESAC.T, CZSRESAC.T, and CZXRESAC.T.
} 
same total energy as did the LBLWRF above ${ }^{36}$. Estimates were provided for all hours within the five-year period, whereas LBLWRF estimates are missing for some hours, because of missing weather data.

These models were developed for PG\&E zones but not for the CEC regions.

\subsection{Methods of Comparison}

The basic approach for the comparisons was to develop, for each model, a prediction of hourly load for each hour of the five year period for which AMP data were obtained. ${ }^{37}$ We then compute the difference or residual between the model-predicted loads and the average loads from the AMP sample (by CEC region or PG\&E zone, as appropriate).

We selected three measures upon which to base our evaluations: (1) daily energy use; (2) maximum hourly load; and (3) 4 p.m. load. Figure 6-1 illustrates how the last two measures were calculated. Note that the hour of the predicted maximum hourly load may be different than the hour for observed maximum hourly load. We perform an hourspecific comparison for 4 p.m. because 4 p.m. is often the hour of PG\&E's system peak load in the summer.

We use boxplots to describe the distribution of residuals for each model and for each of the three measures, separately by season and geographic aggregation. A boxplot is a graphical representation which provides a concise description of a distribution. While a histogram describes a distribution by showing the frequency or relative frequency for set bins defined by the range of the variable, a boxplot, in contrast, describes a distribution by indicating which values of the variable correspond to set percentiles of the cumulative distribution, for example, the first quartile (which equals the twenty-fifth percentile). The most advantageous aspect of a boxplot representation is that it provides for easy side-by-side comparison of distributions.

Figure 6-2 is an example of this form of presentation. Each boxplot is comprised of a box with lower and upper ends at the first and third quartiles of the distribution; a white line inside the box indicates the median of the distribution. Connected to the box are

\footnotetext{
36 That is, the hourly load estimates received were expressed as sector loads. We re-scaled these data to match observed UEC by allocating UEC proportionally to sector loads. This re-scaling and subsequent comparisons may bias results, since sector load tends to increase with time, whereas sample average load does not.

${ }^{37}$ Recall that we did not model cooling loads for Winter, implicitly setting these loads to zero.
} 
"whiskers", which are drawn at the nearest value not further than 1.5 times the interquartile range from the nearer quartile, indicating the spread of the bulk of the data. Points lying outside this range are marked individually (by horizontal lines) on the plot, so that the full range of the data is represented on the plot.

Generally speaking, there are two aspects of goodness of fit represented by the box plot: First, the location of the median load indicates the central value of difference between the predicted and observed values; with fifty percent of the residuals above (in signed value) than this median value, and fifty percent below the median value. Since we are examining residuals, a median value at 0 indicates that the prediction corresponding to this residual matched the observed value exactly. We would thus deduce that the "central tendency" of the predictions is to predict observed values accurately. Second, the height of the box and whiskers indicates how "tight" is the fit in terms of dispersion around th mean, other things being equal, a tight dispersion is better than a wide dispersion of residuals. In an absolute sense, the location of the median is probably more important. On the other hand, if the dispersion of residuals is relatively tight, there may be a systematic bias which can be corrected, thereby moving the mean closer to zero.

Since model performance on peak days is of particular importance for forecasting applications, we make a separate comparison for a handful of system peak load days (in addition to making model comparisons by season). We identified 50 system peak load days based on daily 1985-1989 PG\&E system load data provided by CEC and called these days the Peak Day Subset. A system peak load day was defined by computing the ratio of daily system load to the annual system load for each day of the year and then selecting the days with the fifty highest values of this ratio over the five year period. ${ }^{38}$ Note that as a result, the peak day subset may not contain the 50 highest cooling peak loads. This method of day selection has the advantage of being "external" to the data; that is, the criteria for selection of days is independent of the sample data.

\subsection{Results of the Model Comparisons for CEC Regions}

As indicated on Table 6-1, five models were compared for the three CEC regions: (1) LBL's daily Weather Response Functions and binned load shapes (LBLWRF); (2) LBL's hourly Weather Response Functions (LBLHWRF); (3) LBL's grand, smoothed THI matrix with CEC's daily energy allocation procedure (LBLMAT); (4) LBL's raw, unsmoothed, regional THI matrices with CEC's daily energy allocation procedure (LBLRAW); and (5) CEC's current THI matrix and daily energy allocation procedure

${ }^{38}$ We "standardized" loads in this manner to adjust for the generally increasing annual system load over the five years. 
(CECMAT), Since the last three models rely on the same CEC daily energy allocation procedure, the residuals from the predictions of daily energy use are identical. Accordingly, we report only a single residual for these three models in the comparisons of daily energy use (labelled MATRIX).

Model comparisons are presented in a standardized fashion to facilitate evaluation. A single page of comparisons presenting results for each of the three performance measures (daily energy use, peak hourly load, and 4 p.m. load) for all regions $(2,3$, and 4$)$ for the peak day subset (Figure 6-3). We describe results for the system peak days separately from those for the three seasons. For ease of presentation, the results for the three seasons are discussed here, but the graphic summaries are contained in Appendix C.

\subsubsection{Model Performance for System Peak Days}

Figure 6-3 shows the distributions of residuals among days in the Peak Day Subset (the days with the fifty highest ratios of system to annual load between 1985 and 1989). The plots are arranged so that each column corresponds to a CEC region and each row corresponds to one of the three measurements of prediction accuracy. Note that residuals are not restricted to have mean zero within this subset (since the restriction to mean zero is for all data within a Season or all data in the five-year subset, but not necessarily for any subset thereof).

The matrix-based models tend to underpredict daily energy among the Peak Day Subset substantially, particularly for CEC regions 2 and 3 . This tendency towards underprediction again carries through to the CECMAT, LBLMAT, and LBLRAW predictions of maximum hourly load and 4 p.m. load. LBLWRF (except for CEC region 2) and LBLHWRF also tend to underpredict daily energy among the Peak Day Subset, but give much closer predictions than do the matrix-based models. LBLWRF tends to give the best predictions of maximum hourly load and 4 p.m. load for CEC regions 2 and 3 among the models compared, although LBLHWRF does nearly as well.

We examined model predictions for the two days with the highest ratio of daily system load to annual system load: 18 July 1988, and the following day, 19 July 1988. Table 6-3 lists, for these two days and each CEC region and model, predicted daily load, the residual corresponding to that prediction (in comparison to the corresponding AMP sample average load), and the rank of the absolute value of the residual among those for the approximately 460 summer season estimates generated by the model for the region. The matrix-based approach gives its highest underpredictions on these two peak days (e.g. the biggest undeprediction among 1985-1989 estimates for CEC regions 2 and 3 on July 18, 1988). Both LBLWRF and LBLWRF also show large underpredictions for these 
days, relative to the models' predictions for other days. Predictions are particularly low for CEC region 3 on July 19, 1988.

\subsubsection{Overall Model Performance for Three Seasons}

Figure C-1 shows the distribution of model residuals for CEC region 2 for each of the three measures of model predictions compared (daily energy, maximum hourly load, and 4 p.m. load) by season. The data for each statistic are plotted on the same scale (across season and across CEC regions), so the boxplots may be easily compared. The median daily energy residual for the matrix-based models (which allocate daily energy proportionately to WTHIDD) is below zero for all seasons, indicating a tendency to overpredict energy (recall that the average prediction is necessarily near zero as a consequence of model scaling, see discussion in Section 6.5). For the summer season in particular, the matrix-based models yield some large underpredictions of sample loads. The regression-based approaches, LBLHWRF and LBLWRF, perform about the same, both predict considerably better than the matrix-based approach. The tendency of the matrix-based approaches to overpredict daily energy carries through to the other two characteristics compared (since both of these are generated by scaling relative hourly loads by daily energy estimates). Thus, for maximum hourly load and 4 p.m. load, LBLHWRF and LBLWRF again perform substantially better than the matrix-based approaches (showing relatively small spreads), with LBLWRF performing slightly better than LBLHWRF for most comparisons.

Figure C-2 shows the distribution of model residuals for CEC region 3. Results are similar to those for CEC region 2. Again, the daily energy allocation procedure used for the matrix-based estimates leads to some large underpredictions of daily energy in summer.

Figure C-3 shows the distribution of model residuals for CEC region 4 , which is the mildest of the three climate regions. In this case, the matrix-based approaches perform somewhat better than they did for CEC regions 2 and 3. For example, daily energy predictions for the matrix-based approach are about as good as those for LBLWRF and LBLHWRF for summer. Among the models compared, however, LBLHWRF performs the best for predicting both 4 p.m. load and maximum hourly load. 


\subsection{Results of Model Comparisons for PG\&E Zones}

As indicated on Table 6-2, two models were compared for the three PG\&E zones: (1) LBL's daily Weather Response Functions and corresponding binned load shapes; and (2) PG\&E's daily WRFs with corresponding binned load shapes. The presentation of results follows the structure used for the presentation of CEC results.

\subsubsection{Model Performance for System Peak Days}

Figure 6-4 shows the distribution of model residuals for the Peak Day Subset, for each of the three PG\&E zones. For PG\&E zone R, LBLWRF gives unbiased predictions for these days, whereas PGEWRF tends to overpredict. For PG\&E zone $S$, both models tend to undepredict slightly daily energy and 4 p.m. load. The distribution of residuals for PGEWRF are in most cases only slightly broader, if at all, than the corresponding distributions for LBLWRF.

\subsubsection{Overall Model Performance for Three Seasons}

Figures C-4 through C-6 show the distribution of model residuals for the three PG\&E zones, by season. Recall that LBLWRF necessarily has a residual distribution centered on zero, whereas PGEWRF does not (due to the energy scaling mentioned in Section 6.1 and commented on in Section 6.5).

For PG\&E zone R (Figure C-4), PGEWRF tends to overpredict daily energy, and correspondingly overpredict maximum hourly load and 4 p.m. load. For this zone and for each of the three statistics, LBLWRF yields a considerably tighter distribution of residuals than those from PGEWRF. For PG\&E zone $S$ (Figure C-5), the most notable result is the poor predictions from both LBLWRF and PGEWRF for summer. LBLWRF works slightly better than PGEWRF for spring and fall, primarily because of occasional, large overpredictions by PGEWRF. For PG\&E zone X (Figure C-6), PGEWRF gives unbiased predictions overall for all statistics in spring and summer, with again a tendency to overpredict fall loads. While LBLWRF again gives noticeably higher distributions of residuals than PGEWRF for this zone, the difference in LBLWRF and PGEWRF performance is relatively small, which is somewhat surprising considering the fact that PGEWRF was developed on a small subset of the AMP data.

In summary, LBLWRF gives better predictions than PGEWRF for all zones, with PGEWRF having a tendency to overpredict loads. As noted in Section 6.1, PGEWRF estimates are available for all hours $1985-1989$, which implies that missing weather data 
were filled in some cases; this may give a disadvantage to PGEWRF in our evaluations, since no estimates (and hence no comparisons) were made for LBLWRF on days with missing weather data. ${ }^{39}$

\subsection{Interpretation of Results}

In general, the distribution of residuals from a good predictive model would be centered at zero and be clustered relatively tightly about zero. However, the results of the evaluations in this chapter must be interpreted cautiously, since (1) the location of the distribution (i.e. where it is centered relative to zero) is a consequence of the scaling procedures used, which in some cases restricts the location to be near zero; and (2) model "predictions" are not truly predictions in the purest sense of the word, since the same data used to develop the model was also used to evaluate the predictions (except for the daily energy allocation procedure of the matrix-based models).

\subsubsection{Energy Scaling}

As described in Section 6.1, we scaled the output from each model to correspond to a fixed total energy computed over the five years of AMP data. In practice, energy allocations are based on externally-specified scaling factors, e.g. in the HELM forecast file. ${ }^{40}$ Because of this scaling, the average difference between observed and predicted values for matrix-based model estimates must be zero when averaged across the entire five-year period. That is, the prediction is necessarily on average an unbiased estimate of the observed load, because of the method by which predictions were constructed. This restriction is appropriate because energy scaling is a procedure distinct from the portions of the models we examined. ${ }^{41}$

Standard linear regressions always result in regression residuals which overall have a mean of zero. Since the same data were used to build regression models as to generate predictions, the average residual for each season's LBLHWRF model is zero (e.g., the

\footnotetext{
39 Omitting comparisons for both models on days with missing weather data (or deriving LBLWRF estimates for those days, as was apparently done for the PGEWRF model) may provide a fairer comparison of the models.

${ }^{4}$ If one were forecasting AMP sample averages, forecasts would, of course, be biased high or low (or unbiased) depending the accuracy of the energy estimates used for the forecasts.

41 Since winter season was omitted from our comparisons, however, the mean residual over the days compared may not be exactly zero.
} 
mean residual for 1985-1989 summer season combined for CEC region 3 predictions is zero). Also as a result of the regression technique, the average residual for each season's LBLWRF model are slightly above zero (but not exactly zero, because LBLWRF assigns cool days a load of zero, whereas the AMP sample indicate small nonzero loads on even some of the coolest days). Since the PGEWRF estimates were rescaled to yield the observed AMP sample UEC, mean residuals for this model are also zero, when averaged across the five years (including winter).

Thus, by design, the average model residual for the models compared should be zero or nearly so. Consequently, model predictions should be compared on the basis of (1) the spread of residuals about the mean (e.g., the variance, range, etc.) when comparison are mode over all days; and (2) both location and spread for residuals for peak load days, since this evaluation does not restrict estimates to be unbiased among peak days.

\subsubsection{Cross-Validation}

Our evaluations use the same data to assess models as were used to construct them: (1) the LBL matrix models (LBLMAT, LBLRAW) and LBL daily WRF model with binned load shapes (LBLWRF), and the LBL hourly WRF model (LBLHRWF) were all developed on the basis of 1985-1989 AMP sample data; (2) the PGE daily WRF and corresponding binned load shapes was developed on the basis of 1989 data; and (3) as described above, the energy scaling for all models is restricted to match that in the AMP sample. To the extent that the same data are used for model development as for model assessment, results may be more favorable than if the assessment were conducted out-ofsample.

One approach to address this issue would be to use cross-validation; the is, use one part of the AMP sample to build models and a different part to assess the ${ }^{12}$. While this approach would lead to more robust models from the standpoint of predicting sample loads, it remains a step removed from the ultimate forecasting objective, which is to forecast system, not sample, cooling loads. We return to this issue in Chapter 8.

\footnotetext{
${ }^{42}$ This would be fairly complicated however, and, in our opinion, less important than assessing other aspects of model performance relative to forecasting systemwide cooling loads.
} 


\subsection{Summary}

We have evaluated the performance of a variety of models for forecasting cooling load shapes for CEC regions and PG\&E zones. Within the context of this evaluation, we find that the LBL models developed in this project perform better than previous approaches used by CEC (including those developed previously by LBL for CEC using AMP data) and PG\&E. Between the LBL daily Weather Response Functions and associated binned load shapes and the LBL hourly Weather Response Functions, we find that, while the hourly models yield slightly better results for a few measures of model performance in some regions/zones and seasons (notably 4 p.m. loads), the daily models perform as reliably or better than the hourly models in most cases. Hence we consider the daily models at least as reliable as the hourly models overall. Generally speaking, the models do not perform as well in the more temperate region 4 and zone $\mathrm{X}$. 
Table 6-1. Models Compared for CEC regional Load Shape Forecasting

\begin{tabular}{|c|c|c|c|}
\hline Model & Allocation to Day & Allocation to Hour & Data Source Used \\
\hline LBL daily WRF & $\begin{array}{l}\text { LBL Daily Weather } \\
\text { Response } \\
\text { Functions }\end{array}$ & $\begin{array}{l}\text { LBL Binned Load } \\
\text { Shapes }\end{array}$ & $\begin{array}{l}\text { PG\&E AMP - } \\
\text { region/ Season }{ }^{2}\end{array}$ \\
\hline LBL hourly WRF & LBL Hourly Weather & Response Functions & $\begin{array}{l}\text { PG\&E AMP - } \\
\text { region/ Season }\end{array}$ \\
\hline CEC matrix & CEC Weighted THI & CEC THI Matrix & CEC \\
\hline $\begin{array}{l}\text { LBL smoothed } \\
\text { matrix }\end{array}$ & CEC Weighted TH! & $\begin{array}{l}\text { LBL Grand THI } \\
\text { Matrix }\end{array}$ & $\begin{array}{l}\text { PG\&E AMP - All } \\
\text { regions' }\end{array}$ \\
\hline LBL raw matrix & CEC Weighted THI & $\begin{array}{l}\text { LBL "Raw" region } \\
\text { Matrix }\end{array}$ & $\begin{array}{l}\text { PG\&E AMP - } \\
\text { region' }\end{array}$ \\
\hline
\end{tabular}

1 see Eto and Moezzi (1992) for a description of the development of this model

2. see Chapter 3 and 4 of this report for a description of the development of this model

3. see Chapter 5 for a description of the development of this model 
Table 6-2. Models Compared for PG\&E Zonal Load Shape Forecasting

\begin{tabular}{|c|c|c|c|}
\hline Model & Allocation to Day & Allocation to Hour & Data Source Used \\
\hline $\begin{array}{l}\text { PG\&E daily } \\
\text { WRF }\end{array}$ & $\begin{array}{l}\text { PG\&E Daily } \\
\text { Weather Response } \\
\text { Functions }\end{array}$ & $\begin{array}{l}\text { PG\&E Binned Load } \\
\text { Shapes }\end{array}$ & PG\&E \\
\hline LBL daily WRF & $\begin{array}{l}\text { LBL Daily Weather } \\
\text { Response } \\
\text { Functions }\end{array}$ & $\begin{array}{l}\text { LBL Binned Load } \\
\text { Shapes }\end{array}$ & $\begin{array}{l}\text { PG\&E AMP - } \\
\text { region/ Season' }\end{array}$ \\
\hline
\end{tabular}


Table 6-3. Comparison of Model Performance on Top Two System Load Days 1985-1989', by CEC region

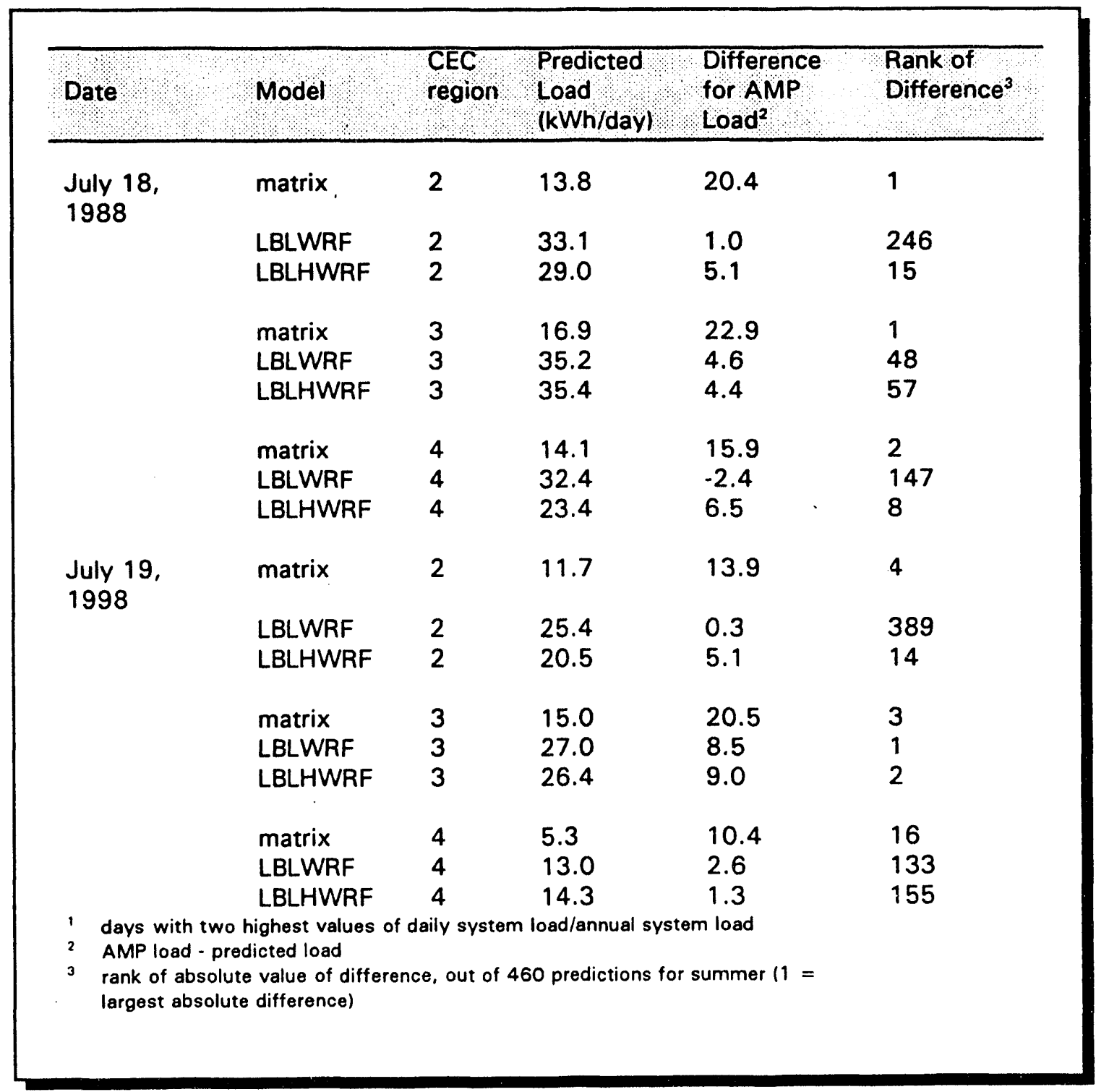


Figure 6-1. Measures of Hourly Fit

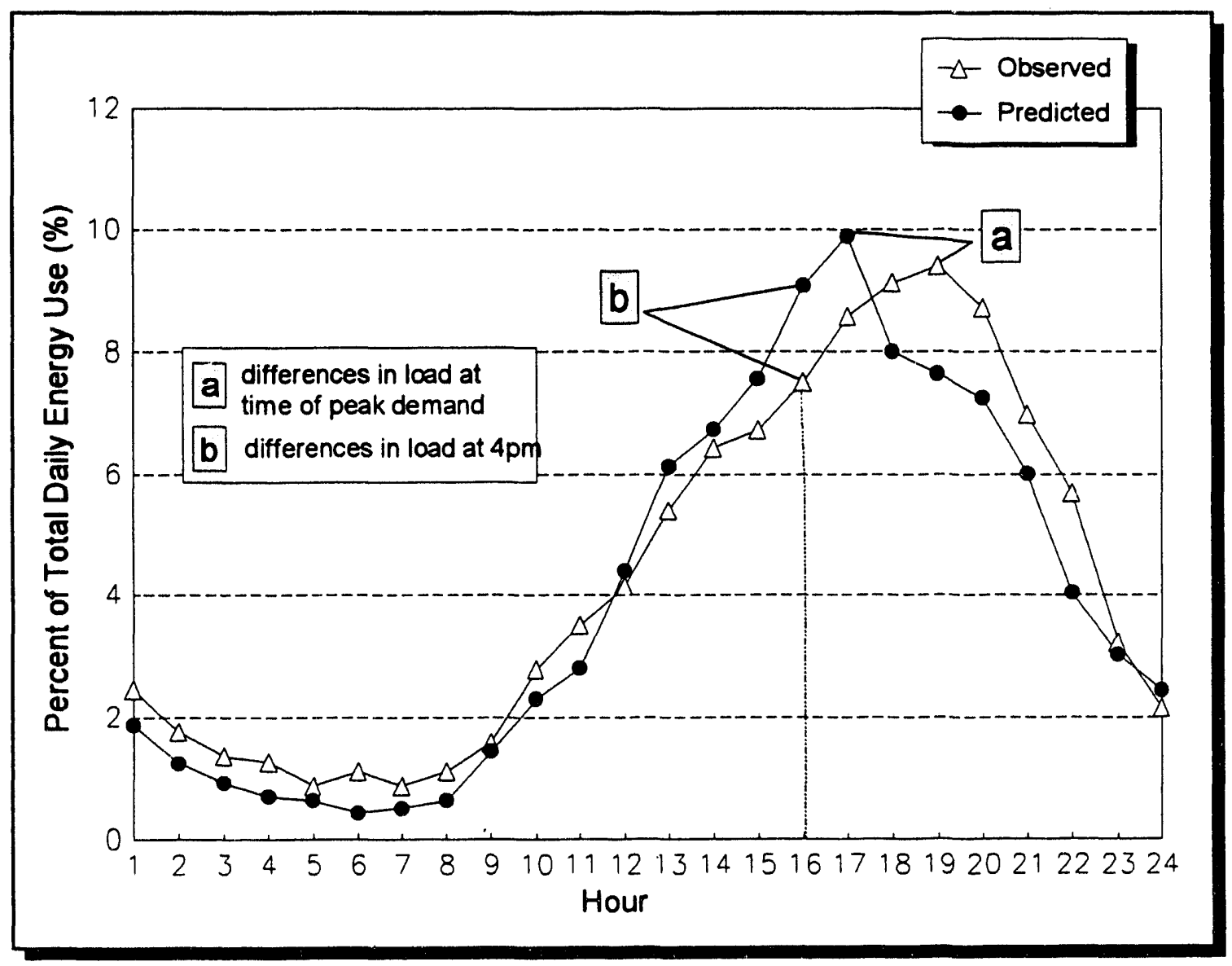




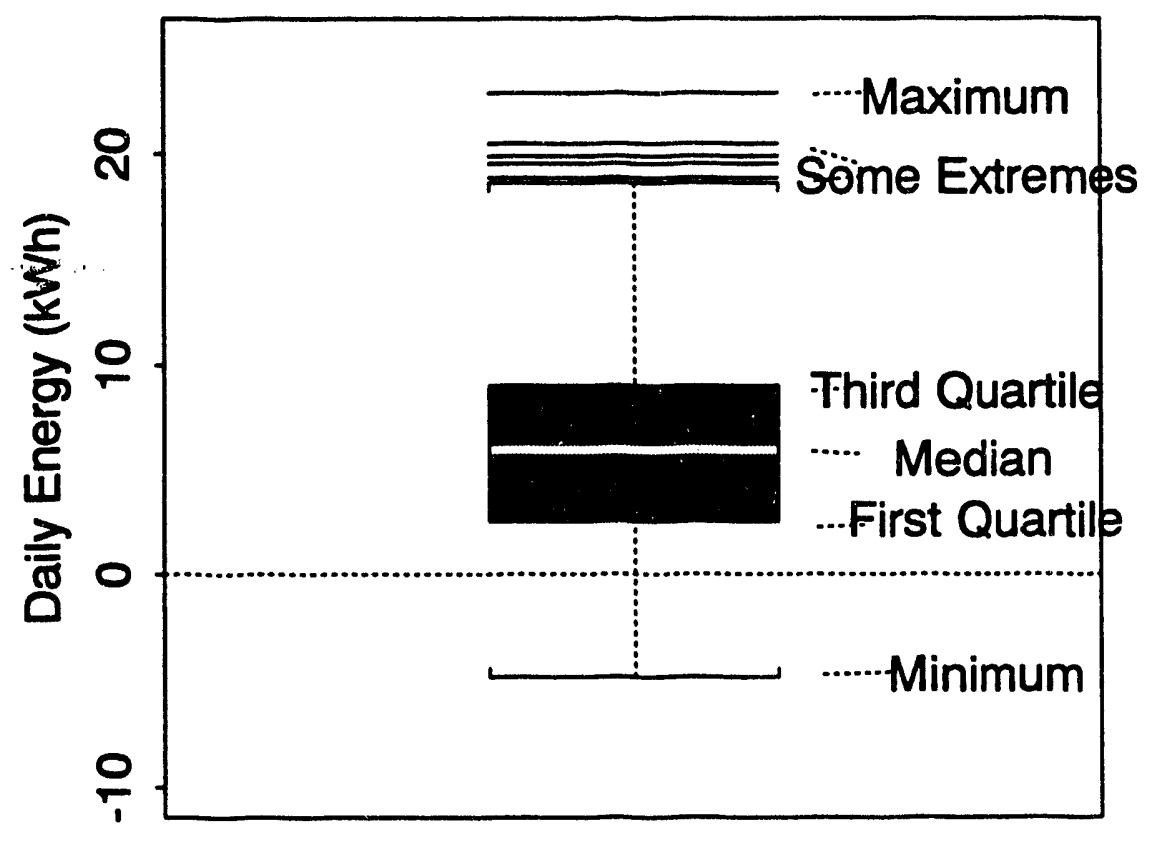

Figure 6-2. Components of a boxplot. 
Region 2
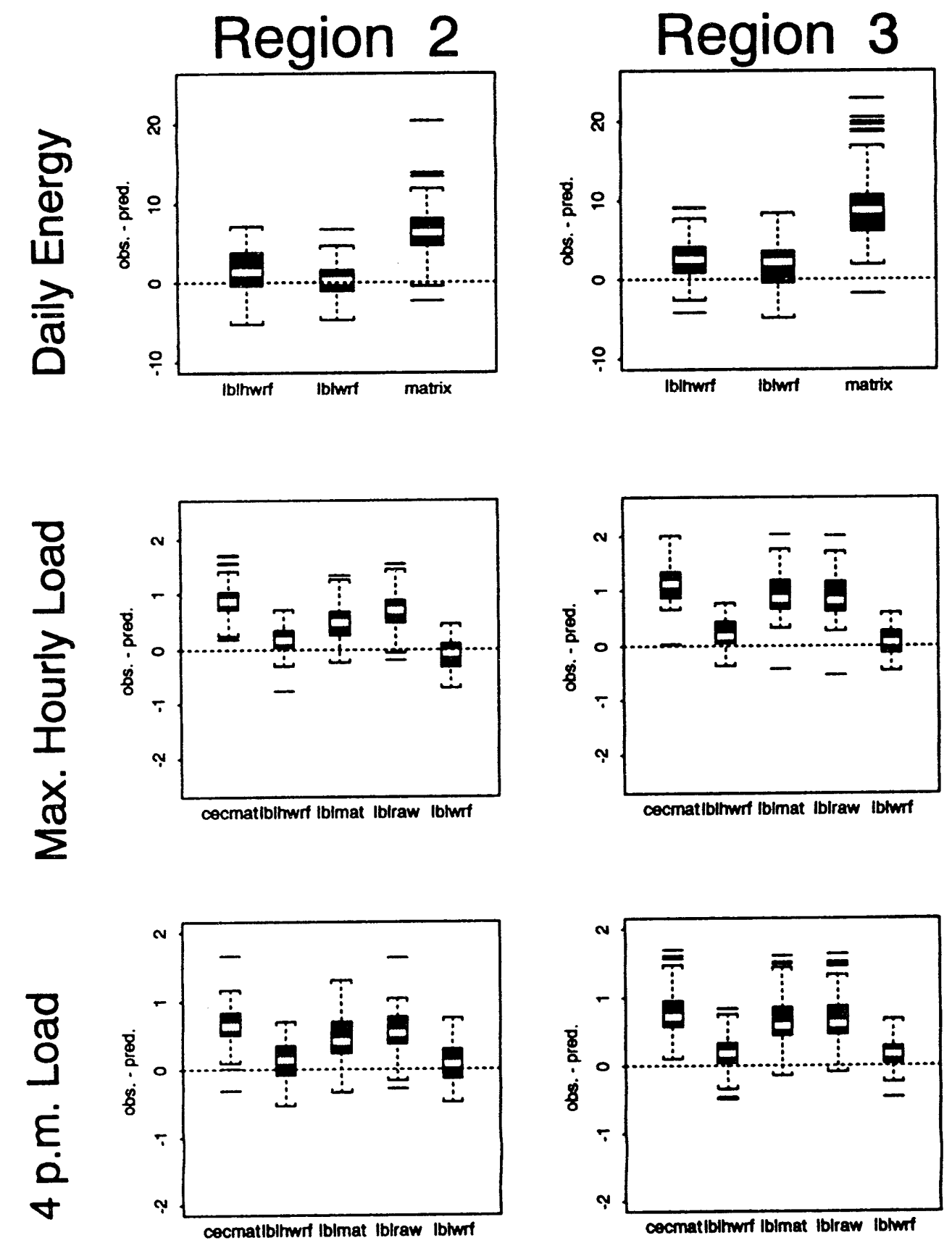
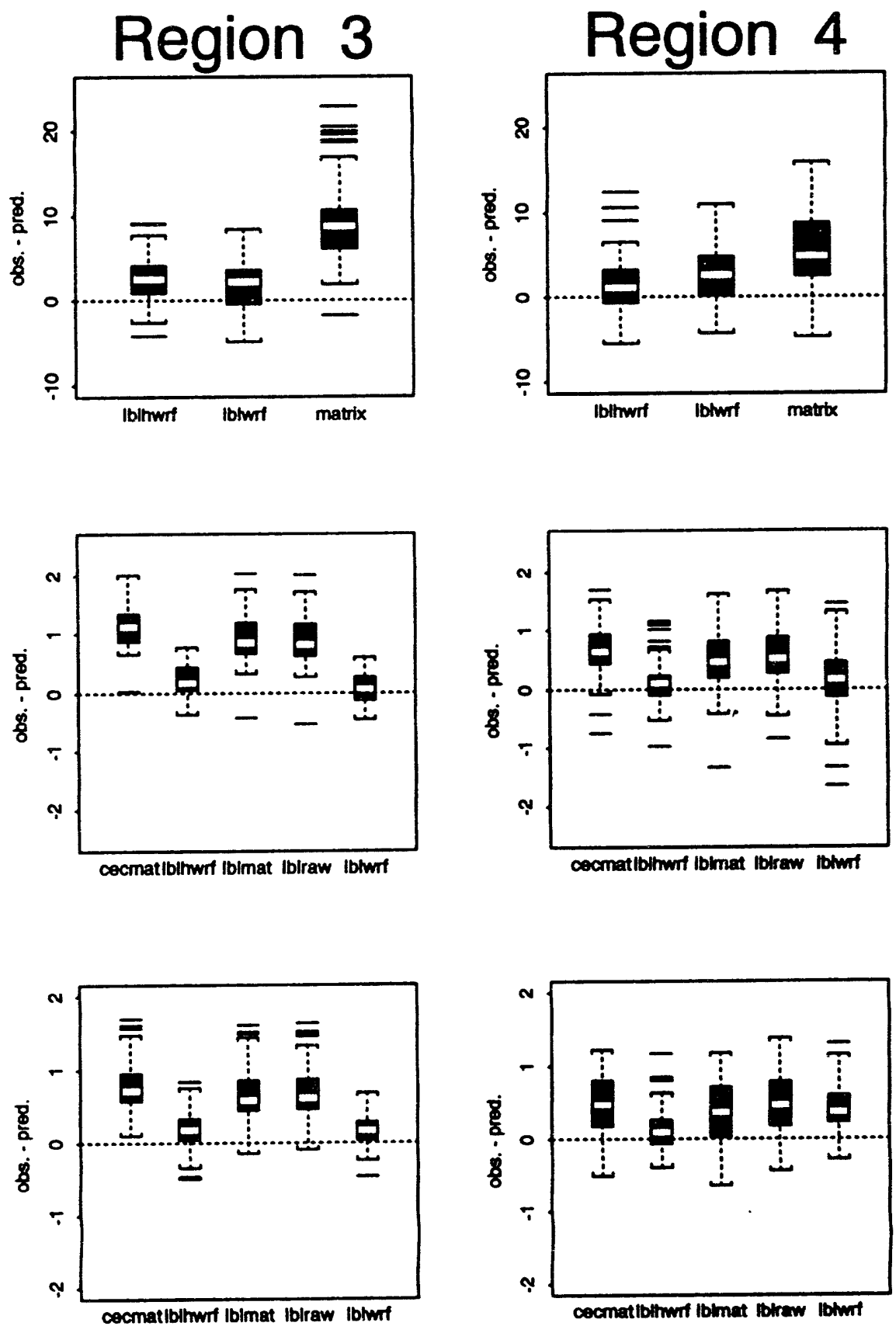

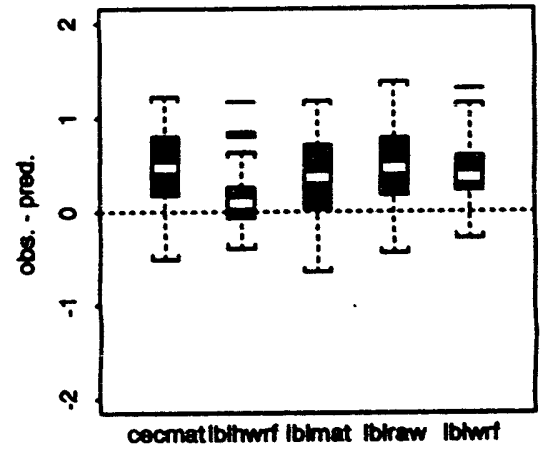

Figure 6-3. Distribution of model residuals (obs. - pred.) for Peak Day Subset by CEC Region. 

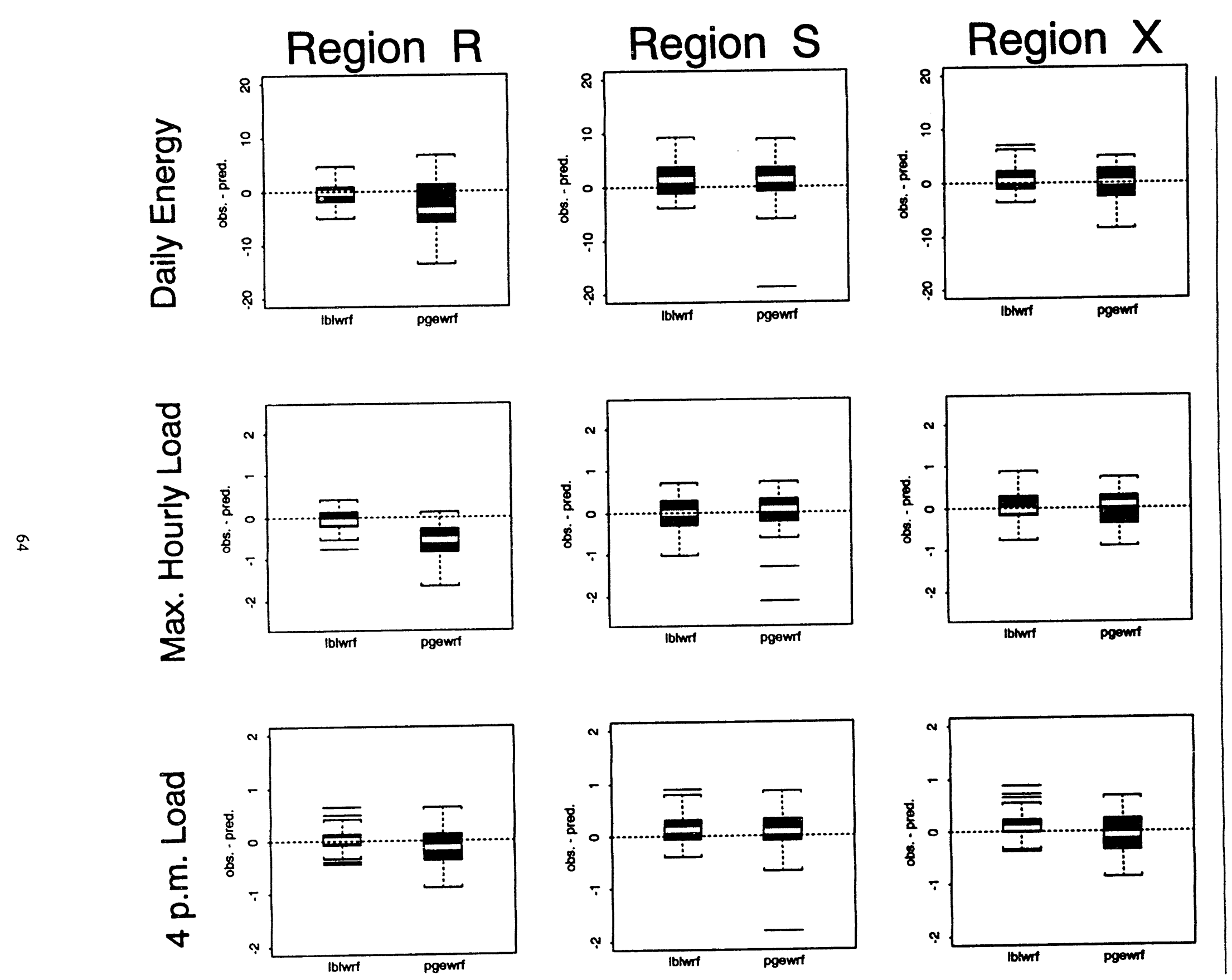

Figure 6-4 . Distribution of model residuals (obs. - pred.) for Peak Day Subset by PG\&E Zone 


\section{Exploratory Analyses}

Chapters 3 through 6 summarize findings from the major areas of research conducted in the project. In meetings held during the analysis process, both PG\&E and CEC identified areas of mutual interest, which were outside the primary focus of research. In this Chapter, we report our findings from exploratory analyses for a few of these areas, including (1) explicit specification of heat storms for modeling daily cooling loads; (2) inclusion of heat pump compressor cooling loads in modeling daily central air conditioner energy use; (3) modeling daily room air conditioner cooling loads; (4) the use of alternative weather data for modeling daily cooling loads in CEC region 4; (5) alternative bin specifications for the hourly load shape libraries; and (6) separate modeling of peak days. Each topic is written in the form of a short, memorandum. Chapter 8 summarizes outstanding issues remaining from these analyses and other areas for future research.

\subsection{Heat Storms}

In order to capture explicitly the additional effects that sustained, extremely hot periods of weather might have on loads, we investigated the possibility of adding an explicit "heat storm" variable to our linear regression models for daily central air conditioner loads. We found some evidence that including a such a variable did modestly improve the load predictions made by daily regression models, but that the effect is probably not large enough to warrant incorporation into forecasting models.

\subsubsection{Motivation}

There is anecdotal evidence that the responsiveness of cooling loads to weather may be different during prolonged periods of hot weather than on isolated hot days. Both thermodynamic (thermal mass) and behavioral explanations have been offered in support of this evidence.

With respect to forecasting, Ignelzi found significant heat storm effects in their linear regression model for Southern California Edison's (SCE) system load. They defined a heat storm as four or more consecutive days with maximum average temperatures greater than 95. Separate variables were included for the sequence of each heat storm in a season (i.e., there were separate variables for the first, second, and any subsequent heat 
storm). The coefficient of each of the three heat storm variables was significant and positive, with the first heat storm having the largest coefficient and the third and subsequent heat storms having the smallest coefficient.

With respect to the daily energy models presented in Chapter 3 , the lagged cooling degree day variables (e.g., CDD95SM1, CDD95SM2 in Table 3-1), the three-day lagged average dry-bulb temperature variable WAVGDRY in PG\&E's daily weather response functions, and the three-day lagged THI variable WTHI-DD in the CEC Peak Demand Forecasting model are, in fact, alternative approaches for capturing heat storm effects.

\subsubsection{Approach}

We considered two issues: first, we examined alternative definitions of heat storms based on a combination of temperature thresholds and duration and determined the number of occurrences of each type for PG\&E zones R, S, and X. Second, for the historic periods of heat storms identified by these definitions, we examined the residuals from our regression models of daily central air cooling loads (Chapter 3).

\subsubsection{Findings}

Table 7-1 shows the number of periods lasting at least four days for PG\&E weather stations in Fresno (zone R) and Sacramento (zone S) and lasting at least three days for the PG\&E weather station in San $\operatorname{Ramon}^{43}$ for zone $X$ in which the average daily temperature was greater than 75, 80, 85, 90, and 95 degrees, for each historic year, 1985 through $1989 .{ }^{44}$ We adopted the convention that a new heat storm began only after the previous heat storm had ended (e.g., a six-day period of temperatures above 80 counts as only one heat storm).

Table 7-2 reports findings for an alternative heat storm definition based on the frequency of four-day (Fresno and Sacramento) and three-day (San Ramon) periods with maximum hourly temperatures (rather than average daily temperature) greater than 90,95 , and 100 degrees.

\footnotetext{
43 There were no heat storms of length greater than three days at San Ramon for the selected temperature thresholds.

44 Days for which temperature data were missing were considered to be below the heat storm threshold.
} 
We investigated the potential explanatory capability (for predicting daily cooling loads) of several of these heat storm definitions by examining the residuals from the final daily WRFs for these zones (summarized in Table 3-4) for each day of a heat storm. The motivation for this approach is that justification for the addition of an explicit heat storm variable should be based on there being a clear pattern in the residuals from the daily energy models during heat storms. That is, such a clear pattern in the residuals would be precisely the phenomena that a heat storm variable would be used to signal (and thereby "explain" by eliminating the residuals).

Figure 7-1 shows, as an example, the distribution of residuals for PG\&E zone $R$ (the hottest region) for three different heat storms definitions: daily average temperature greater than 80 , greater than 85 , and greater than 90 . The first box-plot includes the residuals for all days with temperature below the indicated limit for all summer days from all five years of the data. The second box includes the residuals for all days with temperature above the limit on the given day but below the lower limit on the preceding day. The third box includes the residuals for all days with two consecutive daily average temperatures above the limit and proceeded by a day with an average temperature below the limit. For example, residuals for the third day of a four-day heat storm are in boxplot for length 3, and those for the fourth day are in the boxplot for length 4 . The last box groups together the residuals for all days of a greater than the indicated number $(10,5$, and 3 , respectively).

The first set of box-plots shows that while the distribution is fairly symmetric about zero for the $\mathbf{4 8 6}$ summer days with average dry bulb temperature less than 80 , the regression over-predicts for sustained periods with average dry bulb temperature greater than 80 , except for periods of length at least 10 , when the regression tends to slightly underpredict daily loads. The final set of box-plots indicates that the WRF for very hot days in PG\&E zone $R$ tends to under-predict loads, but does not indicate a heat storm effect beyond this general tendency to under-predict.

These figures suggest that prolonged periods of particularly hot weather in PG\&E zone $\mathbf{R}$ may lead to slightly higher average cooling loads than would be expected on a typical day with similar weather, but the effect, in the context of our PG\&E zone $\mathbf{R}$ Weather Response Function, does not appear to be a large one and might to some extent be thought of as meaning "peak days have higher then predicted loads." Results might be different in the milder zones ( $S$ and $X$ ) or CEC regions (2 and 4). 


\subsubsection{Conclusion}

Our results for the hot PG\&E zone $R$ suggest that adding a heat storm variable to the daily Weather Response Function is not likely to substantially improve daily cooling load forecasts. Because of the importance of predicting and understanding peak day loads, it will remain appropriate to examine heat storm effects, but most likely in conjunction with other possible determinants of peak days. Our previous analyses (in Chapter 6) for example, did indicate a general tendency for LBLWRF, in the comparably hot CEC region 3 to underpredict loads slightly on hot days. This suggests it may be worthwhile to examine this tendency in greater detail. We take up the issue of peak day forecasts again in Section 7.6 and in Chapter 8. 
Table 7-1. Annual Number of Heat Storms for PG\&E Weather Stations, as Defined by Daily Average Dry Bulb Temperature

Fresno: 4 or More Consecutive Days With Average Dry Bulb Temperature

\begin{tabular}{llllll} 
& $>75^{\circ}$ & $>80^{\circ}$ & $>85^{\circ}$ & $>90^{\circ}$ & $>95^{\circ}$ \\
\hline 1985 & 67 & 46 & 8 & 0 & 0 \\
1986 & 82 & 31 & 7 & 0 & 0 \\
1987 & 68 & 24 & 5 & 0 & 0 \\
1988 & 77 & 36 & 21 & 5 & 0 \\
1989 & 58 & 26 & 5 & 0 & 0
\end{tabular}

Sacramento: 4 or More Consecutive Days With Average Dry Bulb Temperature

\begin{tabular}{llllll} 
& $>75^{\circ}$ & $>80^{\circ}$ & $>85^{\circ}$ & $>90^{\circ}$ & $>95^{\circ}$ \\
\hline 1985 & 18 & 3 & 0 & 0 & 0 \\
1986 & 6 & 0 & 0 & 0 & 0 \\
1987 & 14 & 0 & 0 & 0 & 0 \\
1988 & 24 & 6 & 0 & 0 & 0 \\
1989 & 6 & 0 & 0 & 0 & 0
\end{tabular}

San Ramon: 3 or More Consecutive Days With Average Dry Bulb Temperature

\begin{tabular}{llllll}
\multicolumn{1}{c}{} & $>75^{\circ}$ & $>80^{\circ}$ & $>85^{\circ}$ & $>90^{\circ}$ & $>95^{\circ}$ \\
\hline 1985 & 0 & 0 & 0 & 0 & 0 \\
1986 & 0 & 0 & 0 & 0 & 0 \\
1987 & 2 & 0 & 0 & 0 & 0 \\
1988 & 0 & 0 & 0 & 0 & 0 \\
1989 & 0 & 0 & 0 & 0 & 0
\end{tabular}


Table 7-2. Annual Number of Heat Storms for PG\&E Weather Stations, as Defined by Day's Maximum Hourly Dry Bulb Temperature

Fresno: 4 or More Consecutive Days With Maximum Dry Bulb Temperature

\begin{tabular}{llll} 
& $>90^{\circ}$ & $>95^{\circ}$ & $>100^{\circ}$ \\
\hline 1985 & 61 & 34 & 2 \\
1986 & 71 & 30 & 6 \\
1987 & 60 & 22 & 3 \\
1988 & 61 & 32 & 20 \\
1989 & 56 & 24 & 0
\end{tabular}

Sacramento: 4 or More Consecutive Days With Maximum Dry Bulb Temperature $>90^{\circ}$ $>95^{\circ}>100^{\circ}$

\begin{tabular}{llll}
\hline 1985 & 35 & 13 & 1 \\
1986 & 28 & 3 & 0 \\
1987 & 24 & 5 & 0 \\
1988 & 30 & 16 & 0 \\
1989 & 16 & 4 & 0
\end{tabular}

San Ramon: 3 or More Consecutive Days With Maximum Dry Bulb Temperature

\begin{tabular}{|c|c|c|c|}
\hline 1985 & 3 & J & 0 \\
\hline 1986 & 0 & 0 & 0 \\
\hline 1987 & 7 & 2 & 0 \\
\hline 1988 & 3 & 2 & 0 \\
\hline 1989 & 2 & 1 & 0 \\
\hline
\end{tabular}




\section{avg temp $>80$}
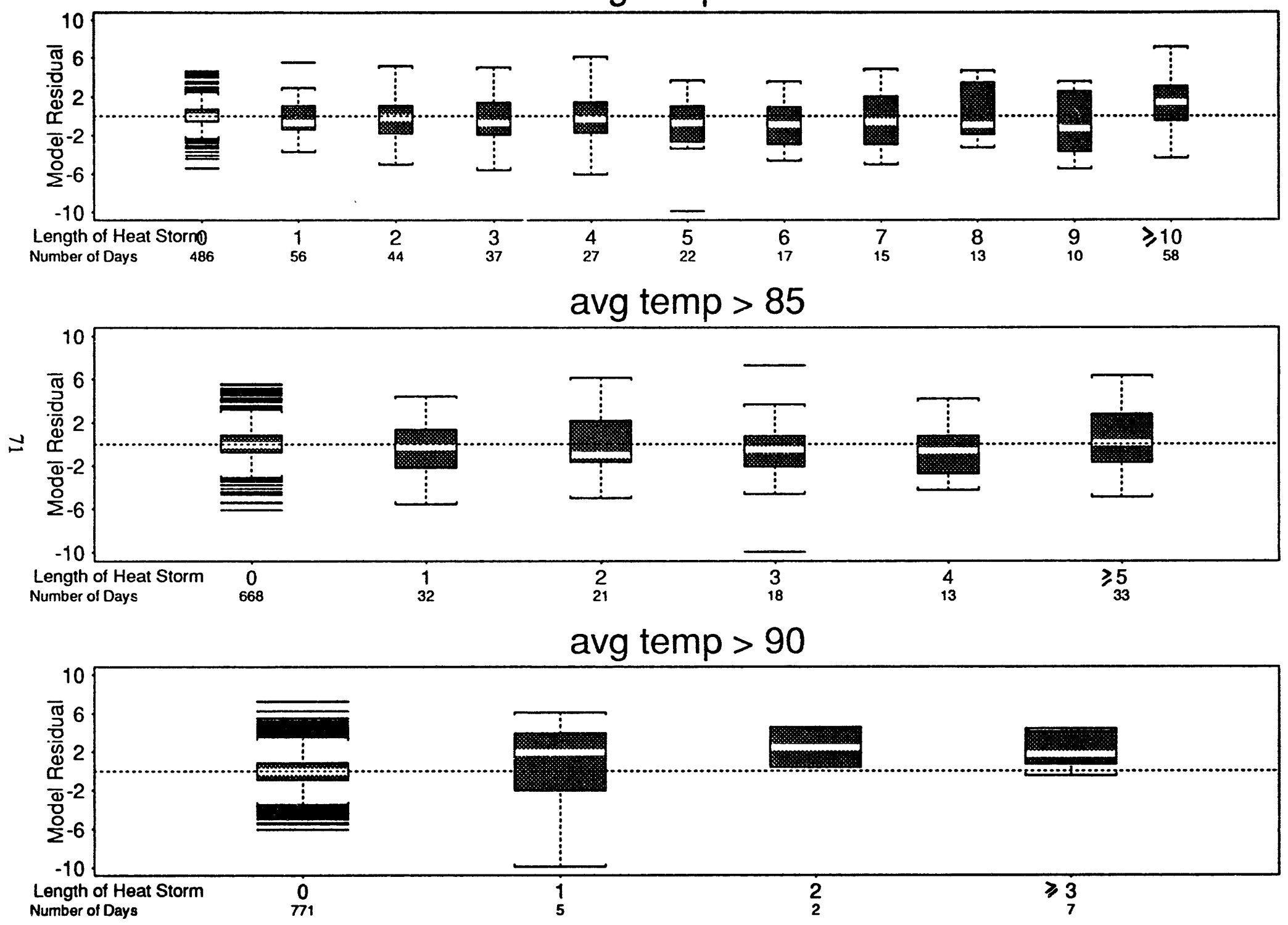

Figure 7-1. Distribution of Residuals from Daily WRF by Length of Heat Storm, PG\&E Zone R. 


\subsection{Heat Pump Compressor}

We investigated differences between heat pump compressor loads and central air conditioner loads in the AMP sample at the level of daily and monthly energy use. We found aggregate loads to be roughly comparable between the two appliance types, adjusting for the difference in average square footage of the residences in which the two different appliance types were metered.

\subsubsection{Motivation}

Heat pump compressors use the same technology to provide cooling as do central air conditioners, but metered data from heat pump compressors cannot simply be included with those from central air conditioners because heat pump compressors provide heating as well as cooling, whereas central air conditioners provide only cooling (save for small heating ventilation loads). Nevertheless, the heat pump compressor data contain potentially valuable information on cooling loads, which may be useful for forecasts. For example, if the use of heat pumps in the cooling mode differs significantly from the use of cooling-only central air conditioners, explicit recognition of these differences in the forecast may be warranted.

\subsubsection{Approach}

A comparison between heat pump compressor and central air conditioner data is complicated by several factors. First, as mentioned, heat pump compressors are used to meet heating loads whereas central air conditioners do not. Second, while over 350 central air conditioners were metered in the AMP sample, less than 50 heat pump compressors were metered; the small sample size for heat pump compressors tends to make population load estimates relatively imprecise. Third, residences with heat pump compressors metered are on average larger than those with central air conditioner metered: for the 1985-1986 AMP participants, the size of the average home with a central air conditioner was 1750 square feet, whereas the average square footage of a home with a heat pump was 2200 (Brodsky and McNicoll 1987) or 26\% larger. Fourth, the total household electricity consumption of homes with heat pump compressors is twice that of average homes (Brodsky and McNicoll 1987).

We limited our analysis to simple comparisons of energy use on a monthly and daily basis. For example, the small sample size for heat pump compressors would made estimation of hourly or daily Weather Response Functions solely on the basis of heat 
pump compressor data impractical. Table 7-3 reports the number of heat pump compressors metered by geographic region. (The comparable counts for central air conditioners are presented in Table 2-1 and 2-2.)

\subsubsection{Findings}

Table 7-4 reports the monthly heat pump compressor UEC (averaged over 1985-1989) for the regions with at least 10 heat pump compressors metered on average (eliminating PG\&E zone $X$ and CEC region 4), along with average monthly central air conditioner UECs. For the summer months, heat pump compressor UECs are very close to the central air conditioner UECs for PG\&E zone S, and about 10-20 percent higher than central air conditioner UECs in PG\&E zone R and CEC region 3; this is consistent with what one would expect, based on the somewhat larger average area of homes with heat pump compressors.

We also compared heat pump compressor loads to central air conditioner loads on a daily basis. Figure 7-2 is a time series plot of the daily difference between heat pump compressor load and central air conditioner load, as a fraction of the heat pump compressor load, for PG\&E zone R and S. Only days with appreciable cooling (central air conditioner load greater than $5 \mathrm{kWh}$ /day are included). The 1987 data shows the greatest difference, but this difference should probably be disregarded since in the copy of the AMP data we used for analyses, half of the expected PG\&E zone $R$ heat pump compressor data is missing. Spikes above the line suggest possible heating. Particularly for PG\&E zone R, there is appreciable variability in the day-tc-day relative differences in heat pump compressor and central air conditioner load. Part of this variability may be due to the small sample sizes for heat pump compressor.

\subsubsection{Conclusion}

For PG\&E zone R and S, differences between heat purnp compressor UECs and central air conditioner UECs are about what one would expect, given differences in the sizes of residences sampled for the two appliances. Comparison on a daily basis shows substantial variation in the differences in sample average loads between the two appliances, in part, due to the likely presence of heating. Without more information on the capacity of the heat purnps and central air conditioners, as well as better identification of heating versus cooling operation, we believe it is premature to either combine heat pump compressor data with central air conditioner data or develop separate cooling WRFs for heat pump compressors. 
Table 7-3. Number of Heat Pump Compressors Metered by CEC Region and PG\&E Zones

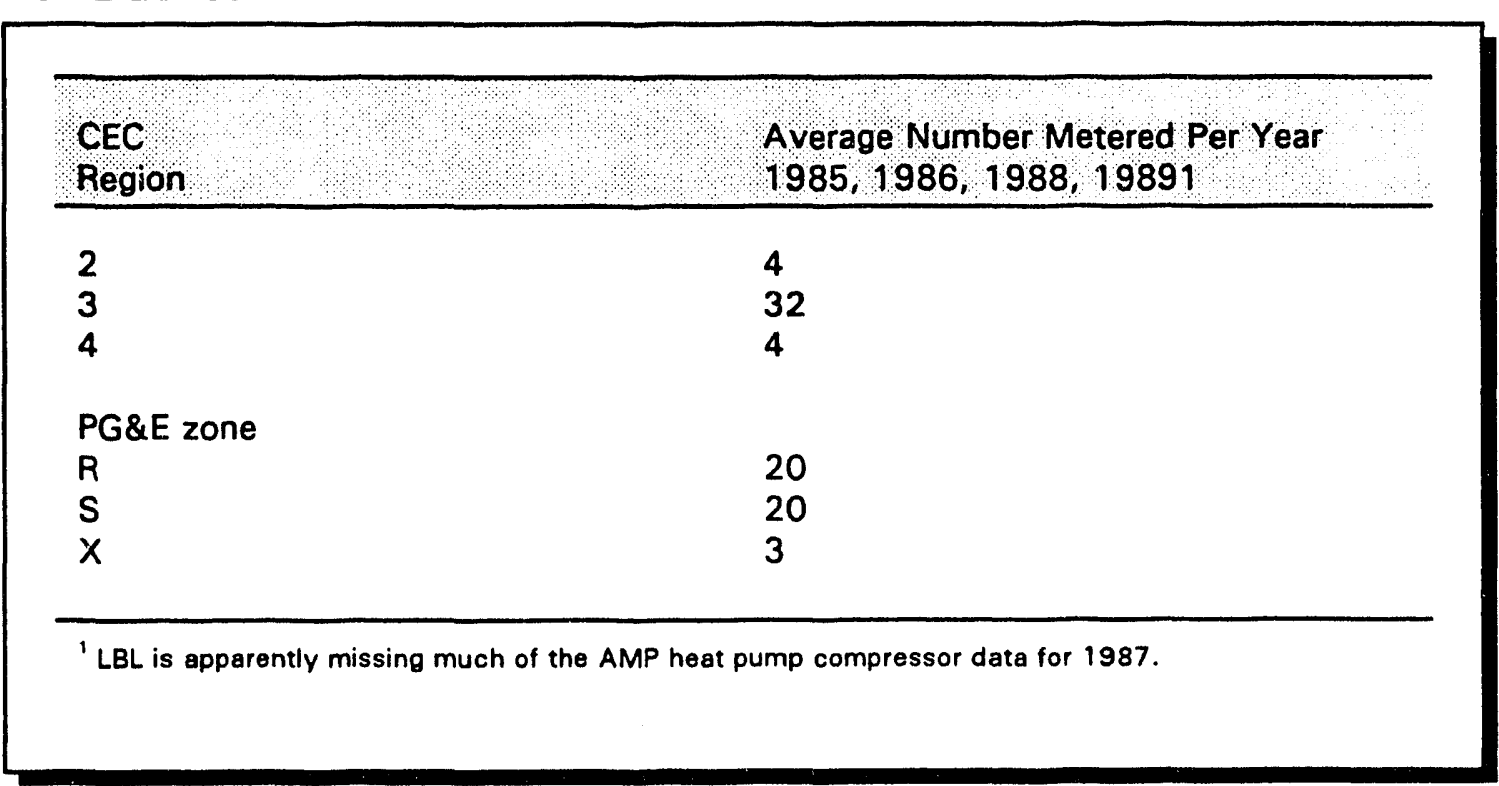

Table 7-4. Average Monthly UECs for Heat Pump Compressor (HPC) and Central Air Conditioner (CAC) for CEC Region 3, PG\&E Zone R, and PG\&E Zone S

\begin{tabular}{|c|c|c|c|c|c|c|}
\hline & \multicolumn{6}{|c|}{ Average Monthly UEC (kWh/month) } \\
\hline & \multicolumn{2}{|c|}{ CEC Region 3} & \multicolumn{2}{|c|}{ PG\&E Zone R } & \multicolumn{2}{|c|}{ PG\&E Zone S } \\
\hline & HPC & CAC & HPC & CAC & HPC & CAC \\
\hline January & 468 & 51 & 435 & 59 & 499 & 44 \\
\hline February & 291 & 36 & 273 & 41 & 311 & 30 \\
\hline March & 231 & 29 & 198 & 33 & 249 & 26 \\
\hline April & 112 & 24 & 108 & 28 & 114 & 26 \\
\hline May & 122 & 82 & 150 & 92 & 110 & 76 \\
\hline June & 273 & 229 & 380 & 336 & 223 & 225 \\
\hline July & 505 & 492 & 688 & 560 & 379 & 366 \\
\hline August & 338 & 378 & 493 & 448 & 232 & 249 \\
\hline September & 163 & 142 & 227 & 173 & 115 & 107 \\
\hline October & $9 ?$ & 34 & 112 & 40 & 74 & 35 \\
\hline November & 219 & 30 & 207 & 33 & 241 & 29 \\
\hline December & 441 & 54 & 438 & 62 & 529 & 53 \\
\hline rce: AMP r & ment 115 & 989) & & & & \\
\hline
\end{tabular}


(Daily HPC - Daily CAC)/HPC, CAC > 5 kWh/day June-Sept 1985-1989, Zone R

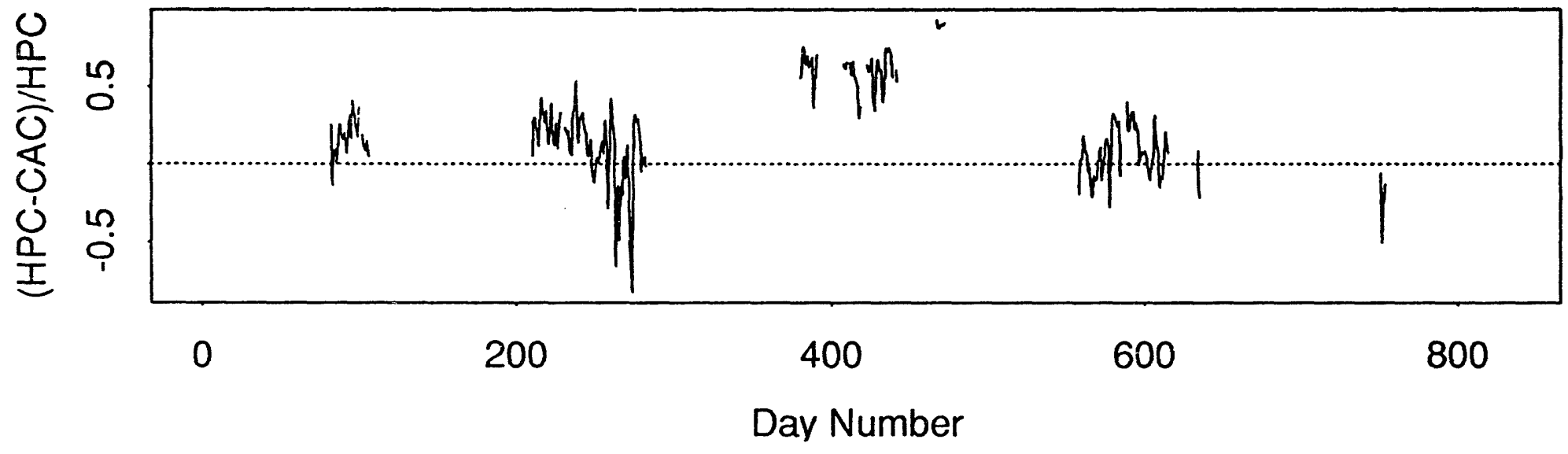

के

(Daily HPC - Daily CAC)/HPC, CAC > 5 kWh/day June-Sept 1985-1989, Zone S

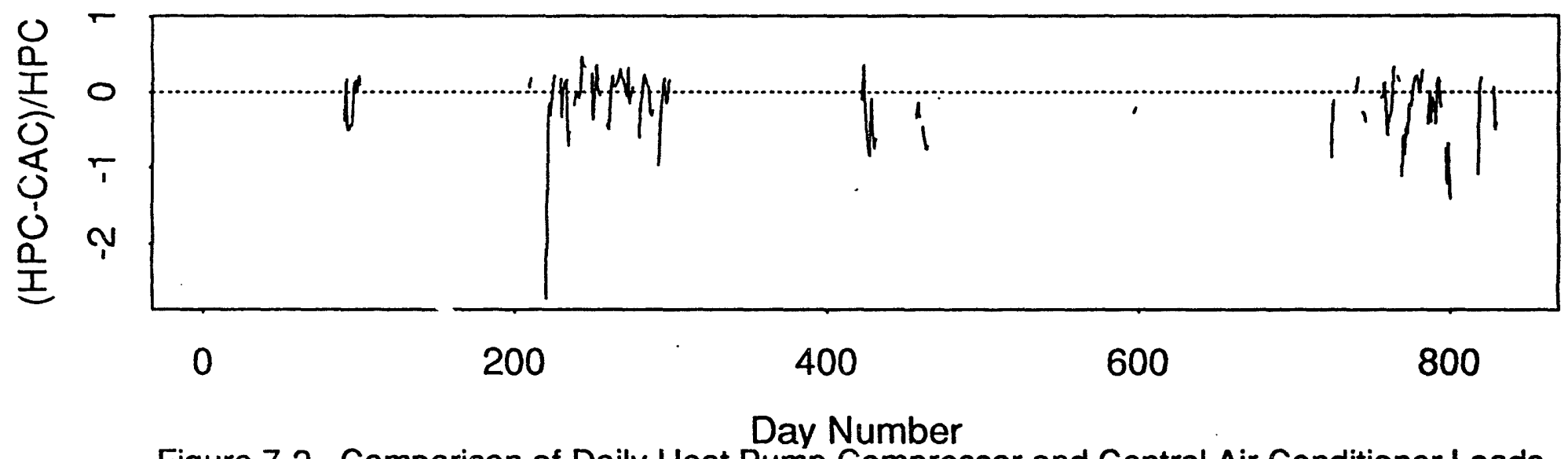

Figure 7-2. Comparison of Daily Heat Pump Compressor and Central Air Conditioner Loads. 


\subsection{Room Air Conditioner}

We developed linear regression models for daily room air conditioner loads for those zones and regions with sufficient data (PG\&E zone $S$ and $X$, and CEC region 4).

\subsubsection{Motivation}

Room air conditioners are estimated to contribute a small but not insignificant proportion of residential cooling loads, with a relatively larger contribution in milder areas. CEC, which forecasts these loads separately from central air conditioners, previously forecast that room air conditioners accounted for 8.4 percent of the combined central and room air conditioner residential cooling loads (excluding cooling loads from evaporative coolers) for all sectors combined, and 5.1 percent, 18.2 percent, and 13.7 percent, respectively for regions 3,2 and all others, respectively. ${ }^{45}$

Relatively few room air conditioners were metered in the AMP sample. As with the heat pump compressors, the small sample sizes tend to make computations based on sample data less reliable. Despite this imprecision, models based on the room air conditioner data alone are likely to perform better than those based on central air conditioner data.

\subsubsection{Approach}

We modeled average room air conditioner load for each of those regions/zones with at least 20 or more metered units using a stepwise variable selection for linear regression based on the same explanatory variables used for the development of the Daily Weather Response Functions for daily central air conditioner load.

\footnotetext{
4 PG\&E estimates an $8.6 \%$ system saturation for room air conditioners, as compared to $24.7 \%$ for central air conditioners and 2\% for heat pumps (Brodsky 1987). In CEC's Revised ER-92 Forecast for 1989 for PG\&E residential air conditioning (single-family, multi-family, and mobile home residences treated separately and then combined), room air conditioners contributed $50.7 \mathrm{GWh}$ as compared to central air conditioner 934.5 GWh in region $3,29.9 \mathrm{GWh}$ (room air conditioner) as compared to $134.1 \mathrm{GWh}$ (central air conditioner) in region 2 , and $42.5 \mathrm{GWh}$ (room air conditioner) as compared to $266.7 \mathrm{GWh}$ (central air conditioner) in "All Other" regions.
} 


\subsubsection{Findings}

Tables 7-5 and 7-6 (taken from Eto 1992) shows 1985-1989 AMP sample average annual and average monthly UEC for room air conditioners for CEC regions and PG\&E zones. Room air conditioner UEC is generally less than half of the central air conditioner UEC, for each of the areas compared. These tables also show the number of metered air conditioners by CEC region and by PG\&E zone. The AMP data base includes metered data for 65 room air conditioners for some portion of the five year period 1985-1989. The metered room air conditioners are concentrated in the cooler geographic areas: for the PG\&E zone aggregations, 36 in zone $\mathrm{X}$ and 20 in zone $\mathrm{S}$, and for the CEC region aggregations, 39 in region 4 , and 13 in region 3.

Table 7-7 summarizes the results of the stepwise-model (again using F-to-enter/F-todelete 0.15 ). indicating which variables were included in the model and the model $r$ squared. Model r-squared for the seasonal models are in most cases lower than the $\mathrm{r}$ squared of the corresponding models for central air conditioner load: 0.72 for room air conditioner model vs. 0.84 for central air conditioner model for PG\&E zone $S$ summer, 0.85 for room air conditioner model vs. 0.91 for central air conditioner model for PG\&E zone $\mathrm{X}$ summer. and 0.78 for room air conditioner model vs. the lower 0.74 for central air conditioner for CEC region 4 summer. The poorer fits for room air conditioner may be partially a result of the relatively smaller sample sizes.

The sets of explanatory variables included in the models for room air conditioner are quite different than those included in the corresponding models for central air conditioner. These differences in variables selected are not necessarily reflective of behavioral differences; as discussed in Chapter 3, caution should be used in drawing conclusions from subsets of variables selected by the stepwise procedure.

However, differences in the specification of the models developed from the central air conditioner data and those developed from the room air conditioner data do not necessarily indicate that a re-scaled central air conditioner model would not be appropriate for modeling room air conditioner loads. To assess whether it would be worthwhile to develop and use separate models for room air conditioner, the prediction errors resulting from using a re-scaled central air conditioner models should be compared to prediction errors from models developed from the room air conditioner data itself. ${ }^{46}$

${ }^{46}$ Units would be re-scaled to reflect room air conditioner UEC instead of central air conditioner UEC. 


\subsubsection{Conclusion}

We developed regression models for daily room air conditioner load for PG\&E zones $S$ and $\mathrm{X}$ and for CEC region 4 using the stepwise regression procedure. The resulting models were somewhat different than the corresponding models for central air conditioner loads. We did not conduct analyses that would allow us to conclude definitively that these models are more appropriate for forecasting room air conditioner loads than is the use of re-scaled central air conditioner models. 
Table 7-5. Annual UEC for Room Air Conditioning, CEC regions

\begin{tabular}{|c|c|c|c|c|c|c|}
\hline APPLIANCE & 1985 & 1986 & 1987 & 1988 & 1989 & $\begin{array}{r}\text { average } \\
\text { (all } \\
\text { years) }\end{array}$ \\
\hline \multicolumn{7}{|l|}{ all regions } \\
\hline mean & 344 & 363 & 476 & 668 & 477 & 475 \\
\hline wt.mean & n/a & $n / a$ & n/a & $n / a$ & 514 & n/a \\
\hline n & 41 & 40 & 50 & 51 & 48 & 46 \\
\hline mean & 371 & 595 & 578 & 754 & 343 & 521 \\
\hline wt.mean & $n / a$ & $n / a$ & $n / a$ & $n / a$ & 325 & $n / a$ \\
\hline std.dev. & 188 & 254 & 262 & 307 & 208 & 261 \\
\hline$n$ & 10 & 9 & 8 & 10 & 10 & 9 \\
\hline \multicolumn{7}{|l|}{ region 4} \\
\hline mean & 296 & 226 & 433 & 502 & 393 & 373 \\
\hline wt.mean & $n / a$ & $n / a$ & $n / a$ & $n / a$ & 381 & $n / a$ \\
\hline std.dev. & 134 & 120 & 474 & 181 & 151 & 275 \\
\hline$n$ & 103 & 98 & 108 & 105 & 120 & 107 \\
\hline urce: AMP m & nts 119 & & & & & \\
\hline
\end{tabular}


Table 7-6. Annual UEC for Room Air Conditioning PG\&E zones

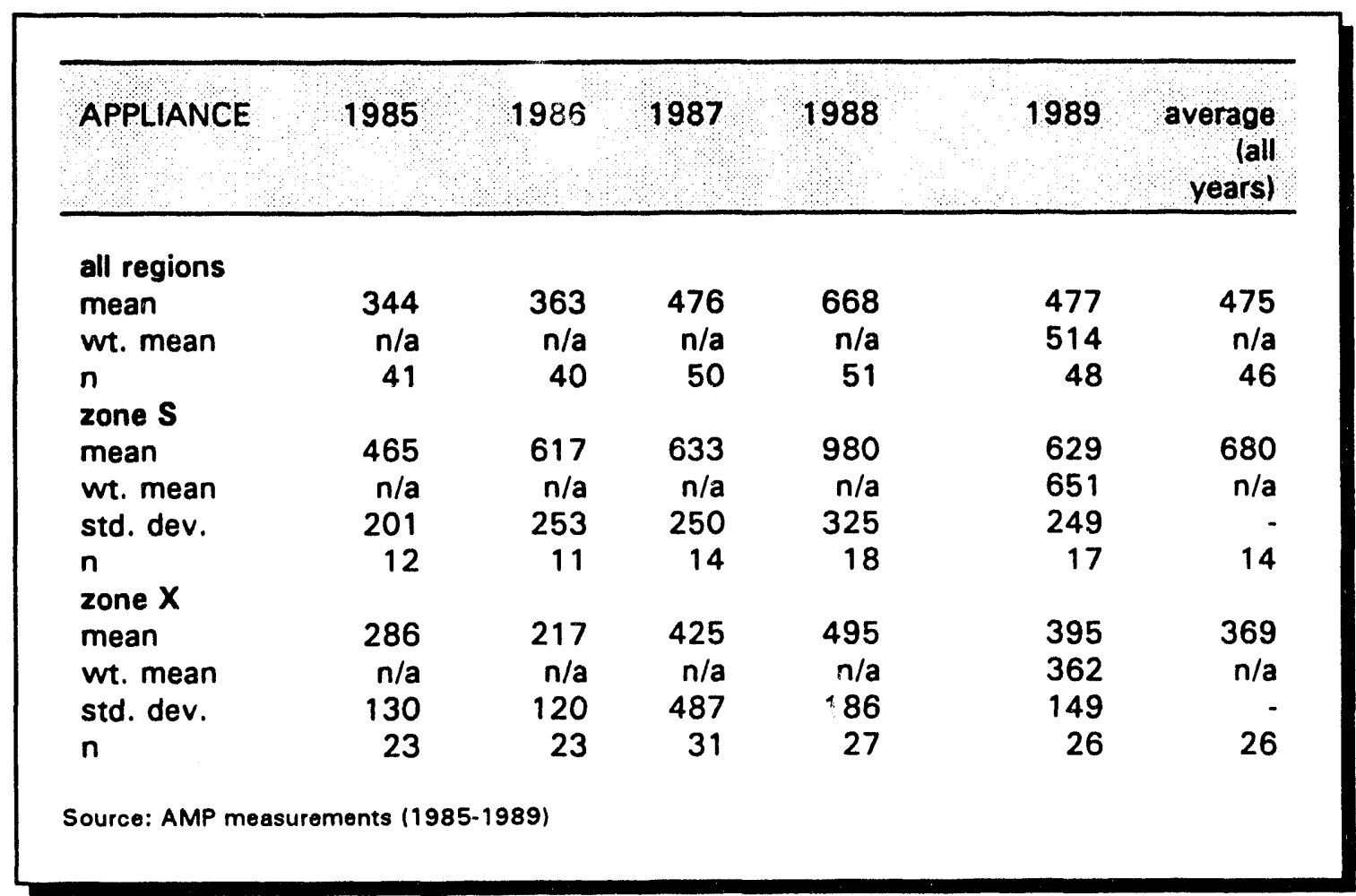


Table 7-7. Summary of Regression Results for Daily Room Air Conditioner Load

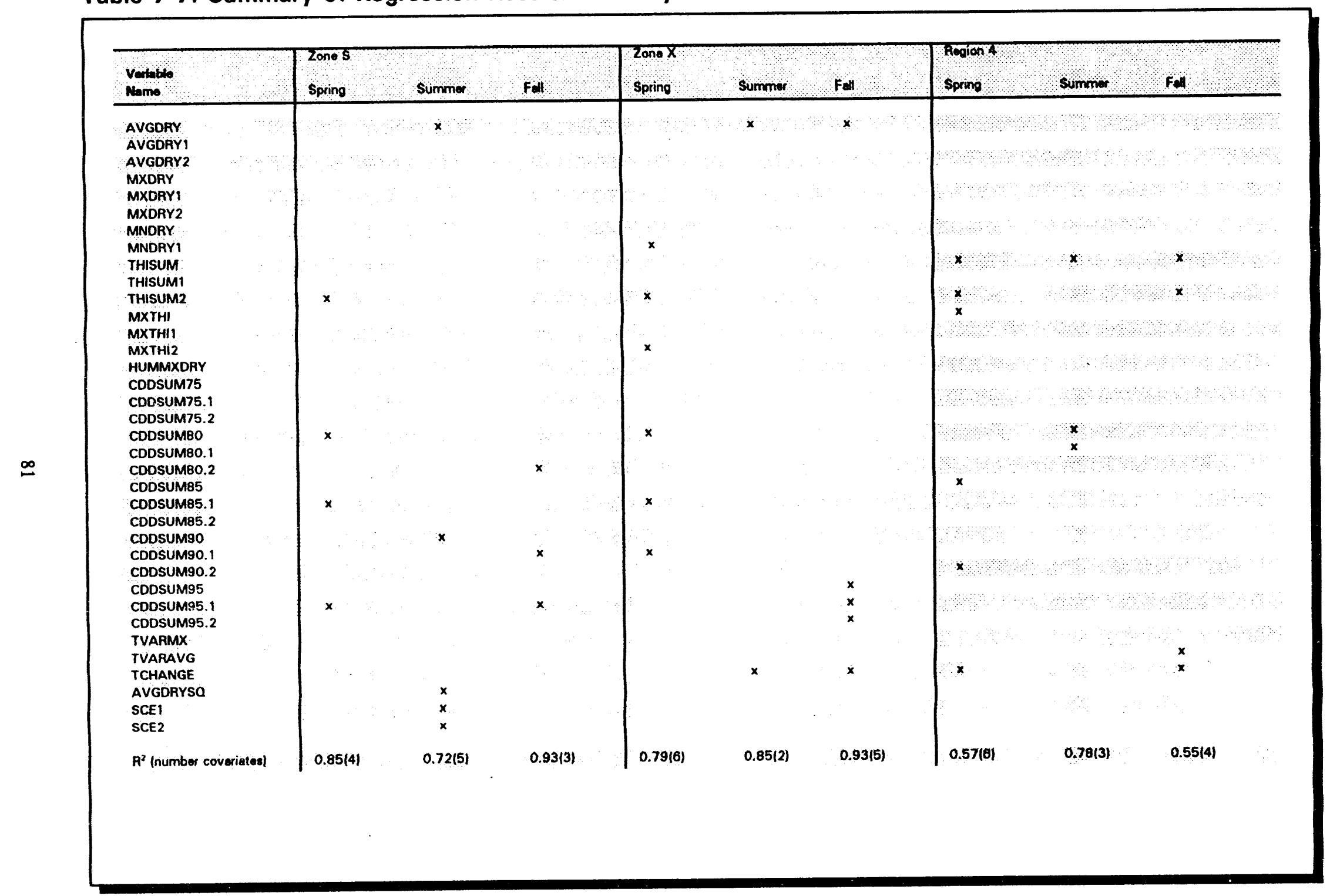




\subsection{Alternate Weather for Modeling CEC Region 4}

We investigated the use of alternative weather data for modeling CEC region 4 daily central air conditioner loads, as a possible means of importing model fit. We found that the weather data used in the original Weather Response Functions reported in Chapter 3 (1985 Sunnyvale, 1986-1989 San Jose) resulted in the highest model $r$-squared of the three sets of weather data tested.

\subsubsection{Motivation}

Both the daily and hourly Weather Response Functions for central air conditioner loads had considerably poorer fits for CEC region 4 than for CEC regions 2 and 3 . For example, the summer season WRF for daily central air conditioner loads in region 4 has an r-squared of 0.74 as opposed to 0.92 for the region 2 model and 0.89 for the region 3 model. The residences metered in that region have, on average, the lowest annual central air conditioner UEC among regions 2,3 , and $4(849 \mathrm{kWh} /$ year in region 4 as compared to 1174 in region 2 and 1651 in region 3; see Table 2-1).

Region 4 also has milder weather than region 2 and region 3, so this relatively poor fit is not unexpected, both because of expectations about customer behavior and because the characteristics of the statistical modeling techniques. In addition, region 4 may be more climatically diverse than the other regions, so that the weather reported at any one weather station may be less representative of the weather at the metered sites.

\subsubsection{Approach}

We used weather data recorded at other sites within region 4 to develop new daily Weather Response Functions. The regression models developed in Chapters 3 and 4 for region 4 were based using four years of weather data from the San Jose station (19861989), and one year of data from the Sunnyvale station (1985) since San Jose weather data was not available for that year. We re-ran the stepwise regression procedure with two alternative sets of weather data: data recorded at the NOAA Sunnyvale station for all five years, and data recorded by PG\&E at its San Ramon weather station. For comparability, regression results are based on 1986-1989 data only for each of the three stations. We used r-squared to compare the fits of the models. We also restricted the number of first-order explanatory variables to six, as required by HELM. 


\subsubsection{Findings}

Table 7-8 shows, for each station, the season-specific r-squared for the linear regressions resulting from the stepwise procedure. R-squared is the highest using San Jose weather for both the summer and fall season. Using San Ramon weather data results in a substantial loss of fit, except for the fall season (r-squared using San Ramon weather is 0.63 as opposed to 0.59 using San Jose weather and 0.53 using Sunnyvale weather). Thus, of the three alternative sets of weather data considered for this region, data from the San Jose station, which was used in developing the WRFs reported in Chapter 3, provide the best fit.

\subsubsection{Conclusion}

Our preliminary investigation into the use of different weather stations indicates that the current practice of relying on San Jose weather remains the best choice for modeling region 4 central air conditioning loads.

Table 7-8. Comparison of CEC region 4 Daily Central Air Conditioner Load Regression Model Fits for Three Alternative Weather Stations

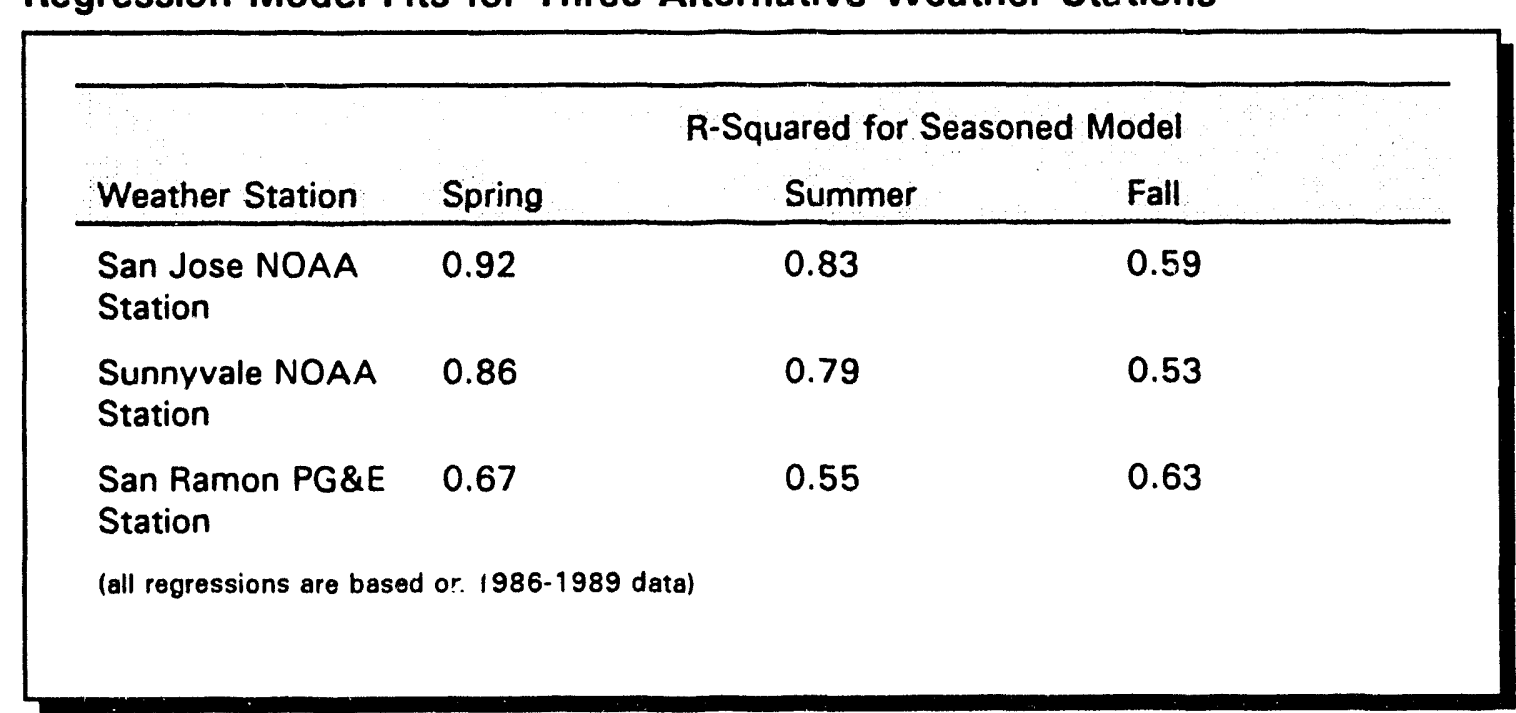




\subsection{Alternative Definitions of Hourly Load Shape Bins}

We examined the variability of loads within the bins previously defined by PG\&E (which were used in the analysis presented in Chapter 4) in order to gain insight into the potential value of alternative bin definitions.

\subsubsection{Motivation}

The definitions for the bins of hourly load shapes developed in Chapter 4 vere adopted from previous work by PG\&E. PG\&E's definitions stemmed from in-house analysis of only 1989 cooling load data. The number and definition of the bins for hourly load shapes stems on the one hand from practical limitations of the HELM model and available weather data, which restrict the total number of bins and the variables eligible for use in defining them. Within these limitations, the primary theoretical issue relates to the observed variability of loads within a bin. As mentioned in Chapter 4, ideally, bins are defined towards maximizing the variability across bins, while minimizing the variability within them. The questions we begin to address in this section are: 1) how well the current binned load shapes represent the load shape for particular days within the bin, and 2) the potential of alternative bin definitions to reduce the variability within bins. The essence of the issue of describing load shape variability within a bin is: how different is a single day's load shape within a given bin likely to be from the load shape used to represent the bin? This difference can be described in many ways; a good parsimonious description should depend on which aspects of the load shape are most important. From a forecasting viewpoint, two important load shape aspects are the maximum hourly load in a day, and the coincident end-use load for the hour of system peak load (4 p.m.).

\subsubsection{Approach}

We first examined the variability of hourly loads within a bin, using the current bin definitions which are specified using AVGDRY. We then explored whether alternative bin definitions, either using AVGDRY or some other weather variable, could reduce the variability within a load shape bin. We evaluated the capability of four different daily weather measures (THISUM, MXDRY and CDDSUM80) to group days using the following procedure: (1) define a univariate description of the load shape; (2) select a weather variable to use in bin definitions; (3) use regression tree analysis with the selected weather variable and load shape variable to determine bin cut-points; and (4) graphically examine the variability of the load shape defined within each bin. 


\subsubsection{Findings}

Figure 7-3 shows the variability in hourly load percentages for each bin for CEC region 3 for each of the six weekday bins (six plots, each with 24 boxes showing hourly load distributions). ${ }^{47}$ The distribution of percentage load for each hour is illustrated by a box; the white bar in the box indicates the median of the distribution, the lower and upper ends of each box represents the first and third quartile of the distribution; the range of the data is indicated by horizontal bars below and above the box; values a factor of more than 1.5 times the interquartile range from the nearest quartile are indicated by detached lines.

The range of 7 p.m. load (typically the peak hour for cooling) for CEC region 3 for weekdays in the hottest bin is between 0.083 and 0.124 , with an interquartile range of 0.095 to 0.108 (57 days). For the next warmest bin, the distribution of 7 p.m. load is slightly "tighter" and somewhat higher, with range between 0.092 and 0.128 , and interquartile range of 0.106 to 0.117 . In general hourly distributions become increasingly dispersed with decreasing temperature. For all bins, the maximum median of the hourly distributions occurs at 7 p.m.

Table 7-9 shows the distribution of the hour in which the maximum load occurred for region 3 summer days, by day-type (weekday vs. weekend), and weather (all days, days with THISUM greater than 100, days with THISUM greater than 200, and THISUM greater than 250). The table shows that the distribution of peak hours is different between weekdays and weekends, but does not change much with increasing THISUM. The weekday peak is typically at 7 p.m. (56.6 percent of all summer days), but is also often at 6 p.m. (27.2 percent of all summer days), with a somewhat higher percentage of 7 p.m. peaks on hotter weekdays ${ }^{48}$. Six p.m. peaks are more frequent on weekends, accounting for 41.4 percent of the peak hours over all weekend days, as compared to 36.8 percent for 7 p.m. This difference in weekday and weekend profiles supports the current practice of developing separating weekdays and weekends in deriving load shape libraries (although results may be different for other CEC regions or PG\&E zones).

Using maximum hourly load as a univariate description of load shape, we examined the use of four weather variables for their capability tc separate load shapes. Current PG\&E

\footnotetext{
${ }^{47}$ Since the hours of the day are considered independently, this depiction does not describe all aspects of load shape variability.

48 Recall that in the convention used by PG\&E, loads are recorded at the end of the period during which they occurred (so that the 7 p.m. load, which is computed by averaging the $6: 30$ and 7:00 p.m. half-hourly loads) is the load between 6 p.m. and 7 p.m.
} 
bins are defined separately by day type (weekday vs. weekend). As previously noted, this practice seems well-justified, so we examined weekday and weekend load shapes separately as well.

We used a technique called regression tree analysis to suggest cut-points to use for binning (Chambers and Hastie 1992). This technique models data by using binary recursive partitioning according to the values of covariates such that at each node the data are split so that the response variable (here percentage load in maximum hour) are maximally distinguished between the two branches of the split. We built trees for each of four weather variables: daily average dry bulb temperature (DRYTMP in Table 3-1), the sum over 24 hours of THIB (see Table 5-1), CDDSM80 (see Table 3-1), and maximum hourly dry bulb temperature (MXDRY in Table 3-1) and used the trees to suggest cut-points by which the load data could be binned.

We first examined the bins defined by partitioning on the basis of DRYTMP. Figure 7-4 shows the distribution of maximum hourly load for each of seven bins which together divide the observed range of DRYTMP, using summer weekday data for CEC region 3. For comparison, Figure 7-4 also shows the distribution of maximum hourly load for PG\&E's existing bin definitions for summer weekday in CEC region 3 . Ideally, the distribution for each bin will be narrow, with relatively little overlap with adjoining bins. The existing PG\&E bins definitions seem to accomplish this distinction as well as the binis suggested by regression tree analysis; this is particularly notable since the new bin definitions were constructed on the basis of maximum hourly load, whereas the PG\&E bin definitions were not.

Figure 7-5 shows the distribution of maximum hourly load for bins defined on the basis of the three other weather variables examined: MXDRY, THISUM68, and CDDSM80. Surprisingly, the bins defined on the basis of MXDRY are the poorest among the weather variables examined. The bins defined on the basis of THISUM68 work quite well, but are not convincingly better than the existing PG\&E bin definitions.

\subsubsection{Conclusions}

Load shapes show a fair amount of variability within bins using the existing PG\&E bin definitions. However, of the three alternative weather variables we used to define load shape bins, none was able to distinguish load shapes significantly better than DRYTMP does for the existing bin definitions. The variable THISUM68 showed the most promise for improving bin definitions. 
Table 7-9. Distribution of Hour of Day in Which Maxirnum Load Occurred for Region 3 Summer AMP Sample Average Load Shapes, by Day Type and THISUM-68 $\left(\sum_{h=1}^{24} \max (\right.$ THI $\left.[\mathrm{h}]-68,0)\right)$

weekday

Per:ent of Days by THISUM-68 Category

All days THISUM-68/100 THISUM-68)200

THISUM-68)250

\begin{tabular}{|c|c|c|c|c|}
\hline \multirow[t]{3}{*}{$\begin{array}{l}4 \text { p.m. or earlier } \\
5 \text { p.m. } \\
6 \text { p.m. } \\
7 \text { p.m. } \\
8 \text { p.m. } \\
9 \text { p.m. or later } \\
\text { number of days }\end{array}$} & $\begin{array}{l}4.6 \\
4.2 \\
27.2 \\
56.6 \\
6.3 \\
1.2 \\
523\end{array}$ & $\begin{array}{l}0.0 \\
3.4 \\
25.5 \\
67.0 \\
4.0 \\
0.0 \\
321\end{array}$ & $\begin{array}{l}0.0 \\
3.2 \\
23.0 \\
70.6 \\
3.2 \\
0.0 \\
187\end{array}$ & $\begin{array}{l}0.0 \\
0.0 \\
39.1 \\
60.9 \\
0.0 \\
0.0 \\
23\end{array}$ \\
\hline & \multicolumn{4}{|c|}{$\begin{array}{l}\text { weekend } \\
\text { ys by THISUM- } 68 \text { Category }\end{array}$} \\
\hline & All days & THISUM-68>100 & THISUM-68)200 & THISUM-68>250 \\
\hline 4 p.m. or earlier & 9.2 & 0.0 & 0.0 & 0.0 \\
\hline 5 p.m. & 4.6 & 3.9 & 6.9 & 0.0 \\
\hline $6 \mathrm{p.m}$. & 41.4 & 47.1 & 41.4 & 50.0 \\
\hline 7 p.m. & 36.8 & 43.1 & 44.8 & 50.0 \\
\hline 8 p.m. & 8.0 & 5.9 & 6.9 & 0.0 \\
\hline 9 p.m. or later & 0.0 & 0.0 & 0.0 & 0.0 \\
\hline number of days & 87 & 51 & 29 & 2 \\
\hline
\end{tabular}


Daily Average Temperature 87.5-100 degrees

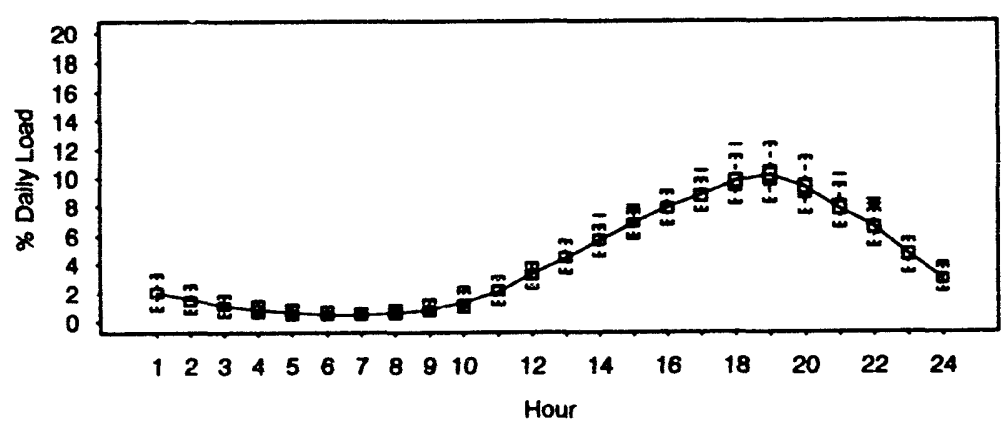

Daily Average Temperature 85-87.5 degrees

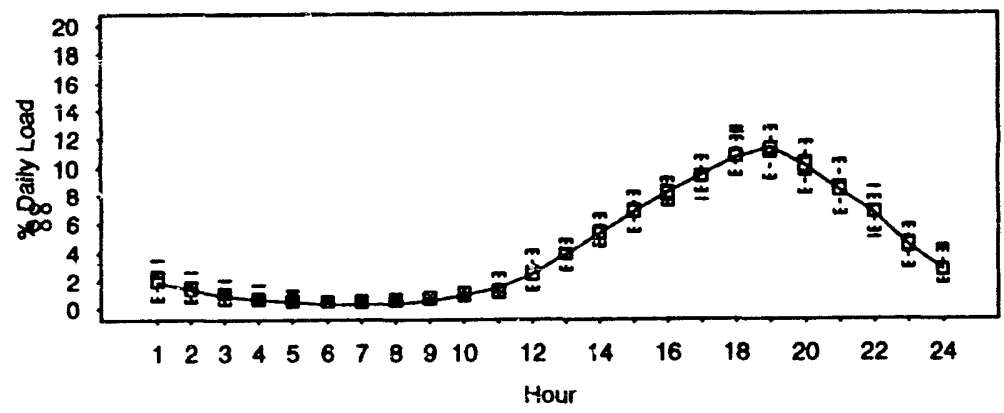

Daily Average Temperature 80-85 degrees

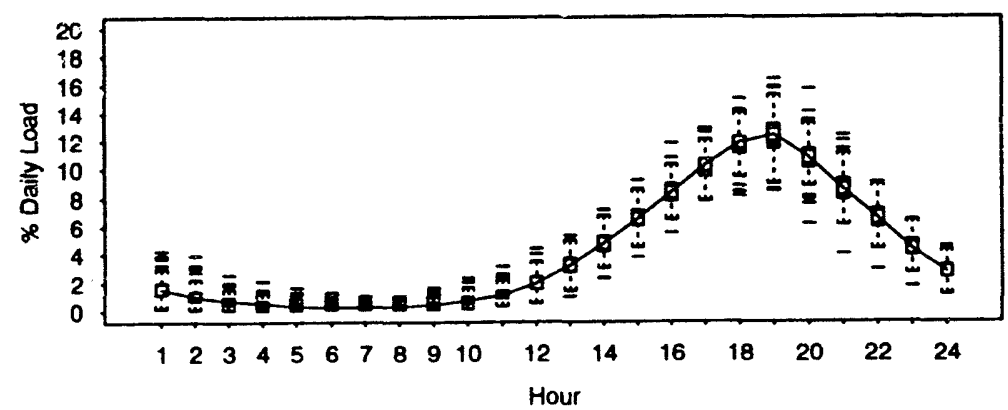

Daily Average Temperature 75-80 degrees

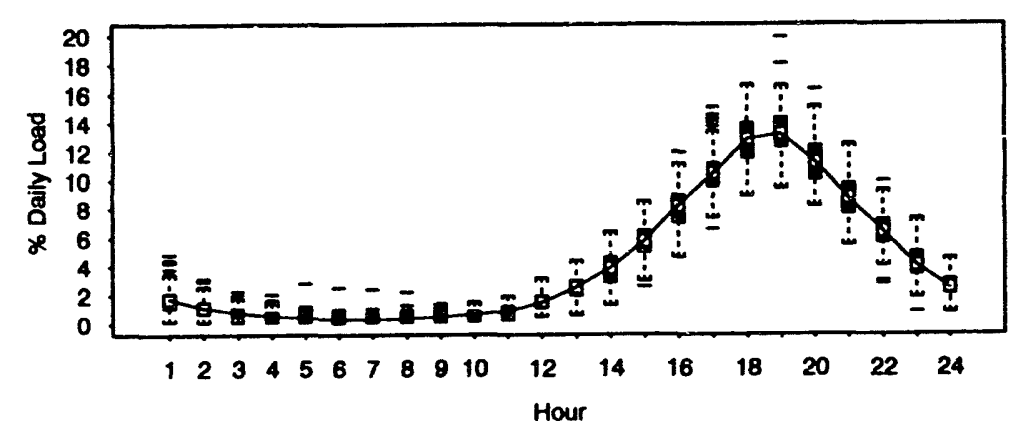

Daily Average Temperature 66.2-75 degrees

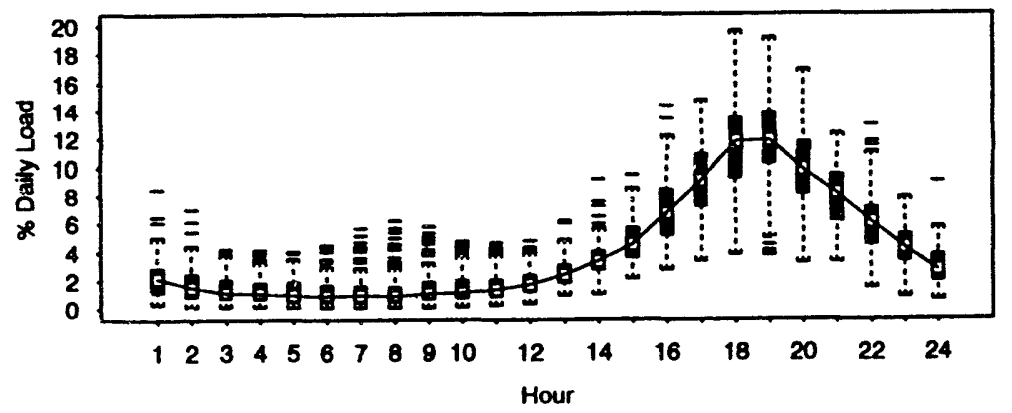

Figure 7-3. Distribution of Hourly Central Air Conditioner Loads by Bin for CEC Region 3 Weekdays. 
Bins suggested by regression tree analysis

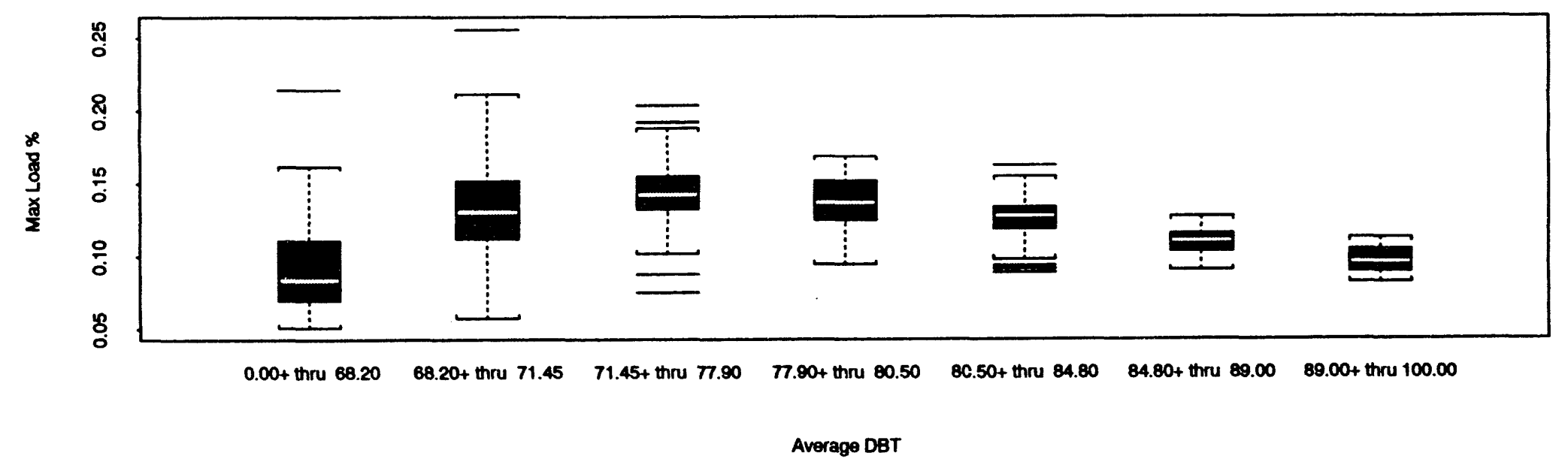

$\infty$

\section{Existing PG\&E bin definitions}

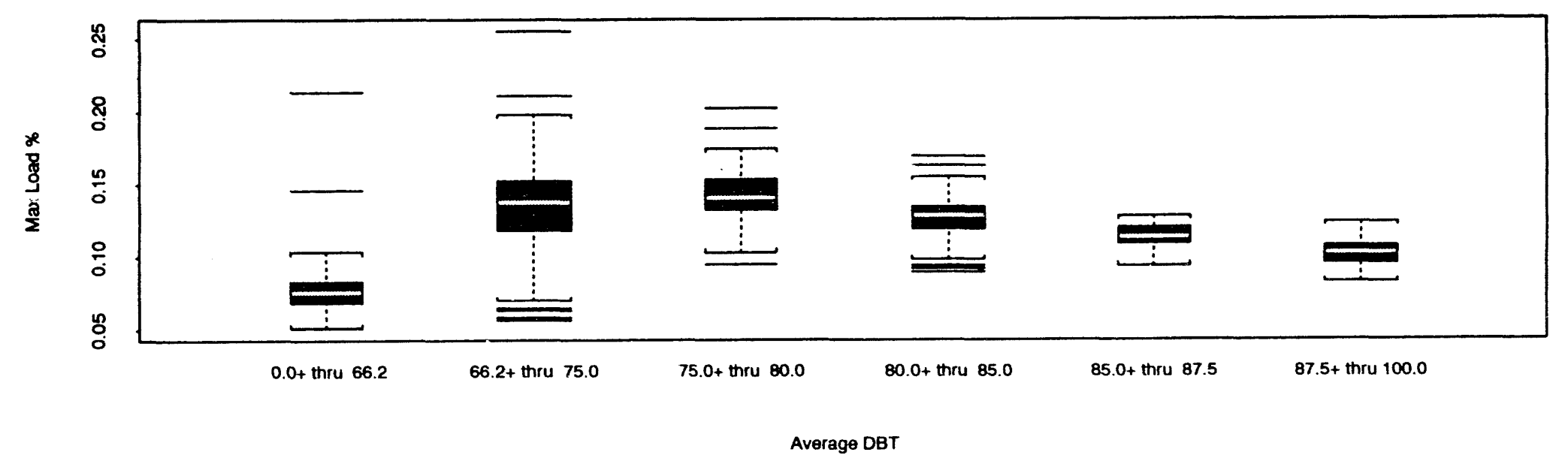

Figure 7-4. Distribution of Maximum Hourly Load for Two Binning Schemes on AVGDRY, CEC Region 3 Weekday. 
Binned by Maximum Hourly Dry-bulb Temperature

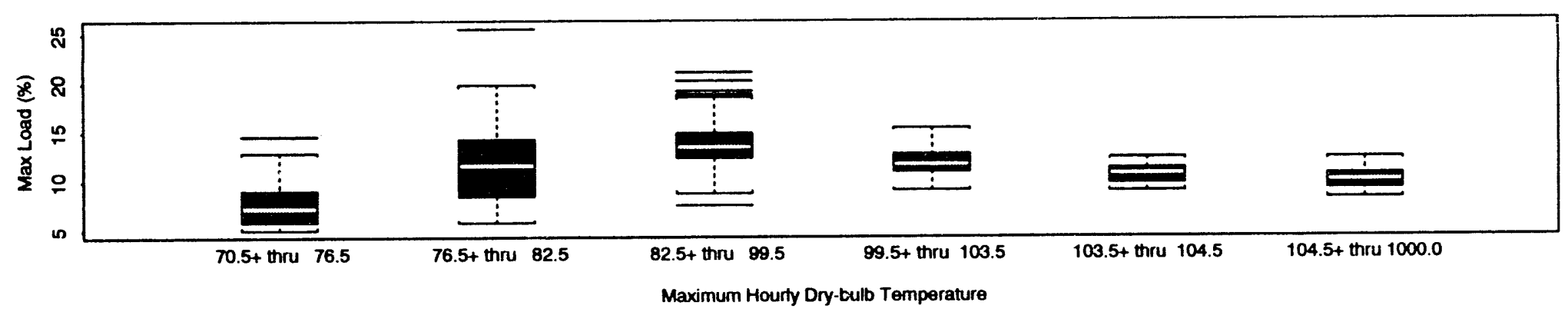

Binned by 24-hour Sum of $\max ($ THI-68,0)

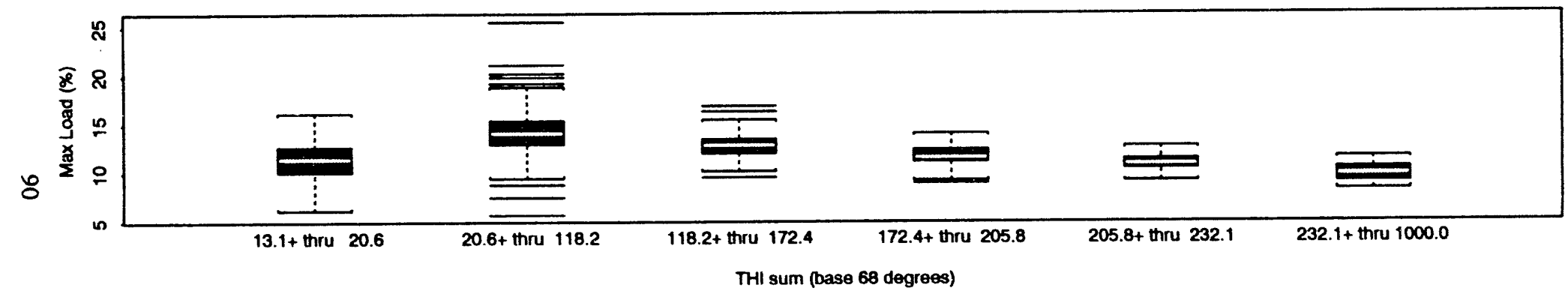

Binned by 24-hour Sum of max(DRYTMP-80,0)

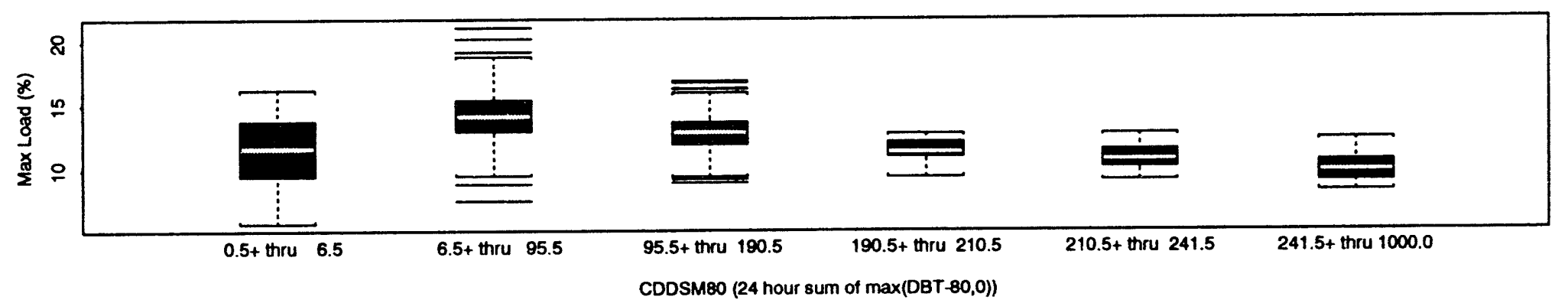

Figure 7-5. Distribution of Maximum Hourly Load for Binning MXDRY, THISUM, and CDD80SM for CEC Region 3 


\subsection{Separate Modeling of Peak Days}

We made an initial assessment of the potential benefits of modeling peak days separately from other days, using summer data for CEC region 3. This assessment is closely related to the analysis of heat storm effects (Section 7.1) and one of our recommendations for future research (Chapter 8).

\subsubsection{Motivation}

Modeling peak day loads is of primary importance in developing load forecasts. Peak days may be different than average days both because they have extreme values, and (from a different perspective) because response to weather on the hottest days may not necessarily be related to weather on cooler days in a straightforward or identifiable manner. In our model assessment phase (Chapter 6), we made separate assessments of performance for the Peak Day Subset of the various models considered in this report, and compared this performance to model performance for all summer days. This comparison indicated that the distribution of residuals for peak days may be considerable different than those for typical summer days.

In the linear regression models we developed, as well as in models used in the past by PG\&E and CEC, peak days and average days are combined. As an alternative, it may be useful to consider modeling peak days separately from average days.

\subsubsection{Approach}

We re-estimated the hourly weather response function Model 3 (see Chapter 5) for each of the 24 hours of the day, using region 3 data for days in the Peak Day Subset, and compared its performance to that of the hourly models developed in Chapter 5 from data for the entire summer season in region 3.

\subsubsection{Findings}

Table 7-10 shows a comparison of the results from some of these hourly models (the 4 p.m., 5 p.m., 6 p.m., and 7 p.m. models) to the corresponding results of the CEC region 3 summer LBLHWRF (LBL's hourly regression models) for the Peak Day Subset. The table shows the Prediction Error within the Peak Day Subset for these models. While this Prediction Error (P.E.) is expected to be lower for the Peak Day Subset 
models, the difference is appreciable, e.g. the hourly 4 p.m. model developed from all summer days yields a P.E. of 5.3, as compared to a P.E. of 3.6 for the hourly 4 p.m. model developed from the Peak Day Subset alone.

\subsubsection{Conclusions}

These results, although not conclusive, suggest that the difference between peak days and average days may warrant separate treatment in the forecasting process.

Table 7-10. Comparison of Prediction Errors in Selected Hours for Model 3 Based on Peak Day Subset to model 3 Based on All Summer Days, for CEC Region 3

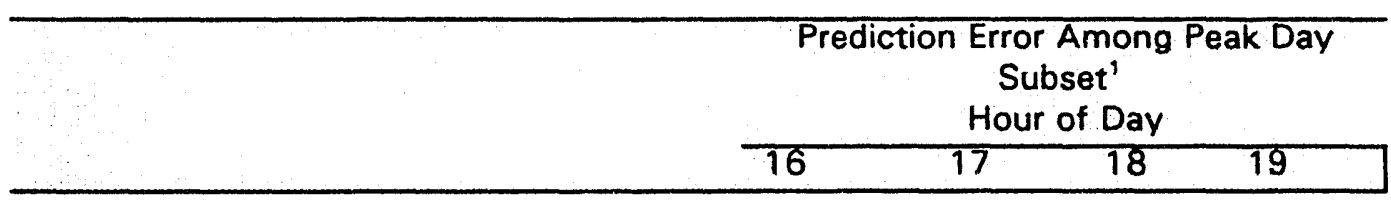

Regression Model:

Load $=f(T H I B$, THIBSO, THIBSUM6, error)

Hourly models based on all Summer days ${ }^{2}$

5.3

5.3

5.4

5.5

Hourly models based on peak day subset ( $r$-squared)

\section{6}

$(0.60)$

3.4

2.5

2.4

1 Peak Day Subset is =days with $50=$ highest $=$ values of Daily Energy/Annual Energy, 1985-1989, based on PG\&E system load data provided by CEC

2 see Table 5-4 for summary of regression results for these models 


\section{Directions for Future Research}

Throughout the analyses undertaken in this project, issues have been identified as candidates for future research. For those that could be readily addressed, albeit in a preliminary fashion, exploratory analyses were conducted and results were reported in Chapter 7. In this chapter, we summarize two particularly important issues remaining from Chapter 7: (1) respecification of binned load shapes (straightforward); and (2) issues underlying the heat storm and peak day analysis (complicated). We also identify two additional, more general issues for future research: (3) representativeness of the AMP sample with respect to the population; (4) potential differences between weather conditions recorded during the sampling period and those used in the forecasting process

Before describing these issues, it is important to preface our comments with a general statement regarding their relative importance. That is, we believe to be that the availability of metered end-use data to support model development represents a tremendous improvement over previous analyses. One should, therefore, view the issues raised as ones which we now have the luxury to address. That is, prior to the availability of these data, the significance of these issues was moot since there was no possibility of resolution.

In establishing priorities for these activities, we believe strongly that the need for additional research must be predicated on a systematic assessment of competing load shape forecasting objectives (such as forecasting system peak, hourly load shapes for 12 typical weeks, minimum load conditions, etc.) in light of the resource constraints faced by the forecasting process.

\subsection{Respecification of Binned Load Shapes}

In Chapter 6 we concluded that the LBL daily Weather Response Functions and binned load shapes generally performed better than did the LBL hourly Weather Response Functions and significantly better than did the other approaches. In Chapter 7, we developed a framework for considering alternative bin definitions for the daily approach. Within this framework we explored the use of a single measure of load shape, maximum peak demand, as a means for creating new bin definitions.

In view of the performance of LBL daily Weather Response Functions, we believe additional work to consider alternative approaches for defining bins is warranted. 


\subsection{Peak Demand versus Load Shape Forecasting}

In several parts of the report, we took up the issue of peak demand forecasting, as distinct from load shape forecasting. In Chapter 6 , we evaluated the performance of our models separately for system peak load days and found that, generally speaking, our models underpredicted loads on these days. In Chapter 7, we evaluated the inclusion of heat storms into the daily energy models and the development of separate models to forecast peak load days. We believe that ultimate resolution of these issues requires directly addressing the tension inherent in forecasting peak demands, after first constraining load shape models with separately-derived annual energy forecasts.

The annual forecasts of energy use that are input into the load shape models represent a constraint on the load shape forecast. For forecasting average load shapes, this constraint is quite reasonable. For forecasting system peak demands, the continuing appropriateness of this constraint should be examined. For example, given a fixed annual energy forecast, allocation of additional energy to peak demand days (either by inclusion of a heat storm effect or separate modeling of peak days) results in less energy being available for allocation to average demand days.

As indicated in the introduction to this section, however, we believe it is appropriate to start this process by first re-visiting the objectives of load shape forecasts in larger context of resource planning to determine the relative importance of (A) system peak demand forecasts, (B) average load shape forecasts, and (C) other issues, such as forecasts of minimum load conditions. Only after the importance of the system peak demand forecast, relative to other applications of the load shape model, is established will it be appropriate to consider further model enhancements to address peak demand issues.

\subsection{Sample Weight Development}

Upon direction from project sponsors, all project analyses have been based on unweighted aggregations of the AMP sample data. There remain un-answered questions as to the representativeness of the sample with respect to the PG\&E population, as represented in the CEC forecasting framework.

We believe that having completed an analysis of the un-weichted data, it is appropriate to examine the potential additional value of including sample weights in subsequent analyses. Specifically, we recommend examining existing sample weights and, if appropriate, developing new sample weights for forecasting purposes. It is likely that 


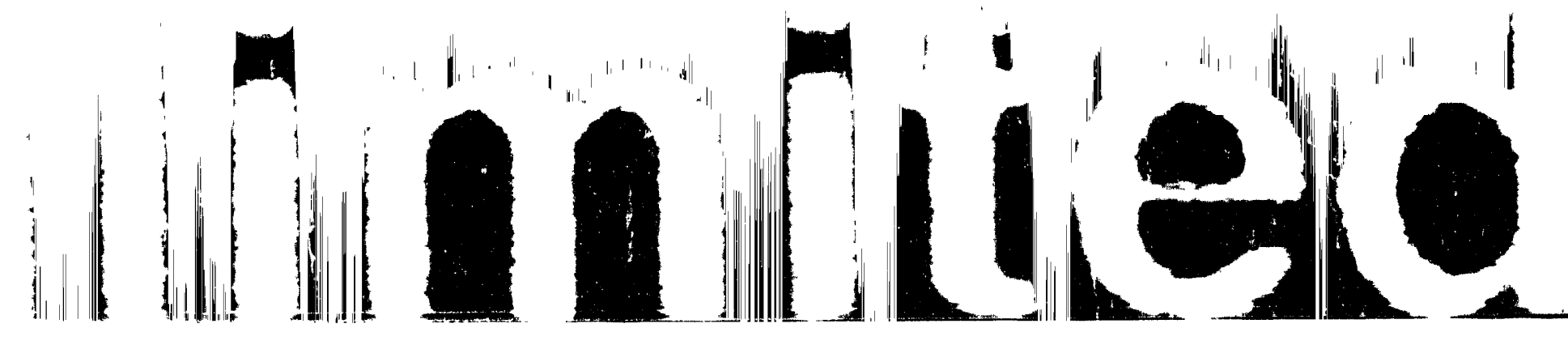

separate sets of sample weights would be required for use by PG\&E and CEC, in view of differences in their forecasting applications.

\subsection{Weather Data for Forecasting}

The weather observed in the metering period is likely to be different from that used in the forecasting process. Several specific issues arise as a result: (A) how similar (or different) is the weather data used in forecasting from that observed in the metered data; (B) to the extent there are differences, what is their significance for the forecasts.

A separate and more general issue is (C) how should weather data developed for forecasting. This issue, in turn, is also related to previously discussed issue (Section 8.2) regarding the relative importance of various load-shape forecasting objectives for system resource planning. 


\section{References}

Brodsky, J. B., and S. E. McNicoll. 1987. "Residential Appliance Load Study, 1985-1986." Appliance Metering Project. San Francisco, CA: PG\&E/Regulatory Cost of Service Department-Research Section. September.

California Energy Commission (CEC). 1991. "California Energy Demand: 1991-2001, Volume II: Electricity Demand Forecasting Methods." CEC Publication P300-91-006. June.

Chambers, J. M., and T. J. Hastie, eds. 1992. Statistical Models in S. Wadsworth \& Brooks/Cole Advanced Books \& Software:Pacific Grove, CA.

Eto, J. H., and M. M. Moezzi. 1992. "Analysis of PG\&E's Residential End-Use Metered Data tc Improve Electricity Demand Forecasts." Berkeley, CA: Lawrence Berkeley Laboratory. LBL-32118. June.

ICF Resources Inc. 1992. "The Hourly Electric Load Model (HELM-PC), Personal Computer Version 2.0 (Beta)." User's Guide. Fairfax, VA: ICF. EPRI Research Project 2863-9. December.

Ignelzi, P. C., and R. E. Way. 1988. "Analysis of Factors For Peak Load Weather Normalization." Final Report. Berkeley, CA: Pacific Consulting Services. Prepared for Southern California Edison Electric System Planning. November (Revised February 1989).

Jaske, M. R., and S. W. Paige ed. 1979. Technical Documentation of the Peak Load Forecasting Model. California Energy Commission:Sacramento, CA.

Kristov, L. 1991. "California Weather Index Development." California Energy Commission (CEC), Demand Forecasting Office. Contract Number 199-066 CA. November.

Pacific Gas and Electric Company. 1991. "Common Forecasting Methodology 9." Revised ER 92 Electric Demand Forms: 1991-2011. December. 
(1) 


\section{Data Handling Conventions}

Prior to our analysis, we developed a number of conventions for handling data. These included the assignment of iridividual households to the CEC and PG\&E geographic regions or zones used in our analysis, the aggregation of half-hourly loads to hours and the alignment of these loads with the weather data, and definition of day types and treatment of missing values. ${ }^{49}$

\section{A.1 Geographic Coding}

We used two distinct sets of geographic aggregations: a set of five climate regions used by CEC, and a set of four climate zones used by PG\&E. Table A-1 shows the number of central and room air conditioners metered for each geographic aggregation. Several regions do not have significant numbers of metered data (e.g., in the case of central and room air conditioning, CEC region 1 and 5, and PG\&E zone T); correspondingly we used only three of CEC's climate regions (regions 2, 3, and 4) and three of PG\&E's climate zones (zones $\mathrm{R}, \mathrm{S}$, and $\mathrm{X}$ ). We refer to these geographic aggregations as CEC regions and PG\&E zones, respectively. Maps of the geographic aggregations are included in Chapter 2.

To make CEC region assignments, we used the zip code of the residence (which PG\&E provided to LBL in the file LBLID.DAT) and then selected a CEC region according to the zip code/climate region correspondence given to us by CEC in the file ZIPzone.DAT. We reviewed these initial assignments by comparing the county of each zip code as given in ZIPzone.DAT to the county as given by U.S. census and postal information to determine cases for which there was a discrepancy in county coding. Based on zip code location and our inspection of the CEC map of geographic climate zones, reassigned residences in 41 zip codes.

Each residence was also assigned to one of four PG\&E zones, R, S, T, or X with corresponding PG\&E weather stations Fresno, Sacramento, Salinas, and San Ramon. These assignments were made according to the LBLID-zone assignments provided by PG\&E in the file CLIMzone.LBL.

\footnotetext{
${ }^{49}$ LBL reviewed some of AMP data for an earlier project (see Eto and Moezzi 1992)
} 


\section{A.2 Weather Data}

We used two sets of weather data in this study, one set derived from NOAA weather data, and a second set of data from PG\&E weather stations. Each CEC region is associated with a NOAA weather station. Each PG\&E zone is associated with a PG\&E weather station. See Table 3-1.

CEC provided LBL with a weather data set derived from NOAA weather files. This data set consists of hourly measurements for 1985-1989 for each of several weather variables at six weather stations: Blue Canyon, Fresno, San Jose, San Francisco, Sacramento, and Sunnyvale. The weather variables reported in this data set are: wet-bulb temperature, dry-bulb temperature, dew point temperature, relative humidity, wind speed, and cloud cover (not all of which were used in our analyses). Other meteorological measurements are reported in the NOAA weather files but were not used in our analyses. According to NOAA documentation, hourly weather data are observed within ten minutes to the hour for which the data are reported. The Blue Canyon weather station is assigned to CEC region 1, the Sacramento weather station is assigned to CEC region, the Fresno weather station is assigned to CEC region 3, the San Jose (1986-1989) and Sunnyvale (1985) weather stations are assigned to CEC region 4, and the San Francisco Airport weather station is assigned to CEC region 5 (but CEC regions 1 and 5 were not analyzed in this study).

Each of the annual PG\&E weather data sets we received for 1985-1989 contains halfhourly measurements of dry-bulb temperature and relative humidity for 25 weather stations. In this report we use data from only three of these stations, Fresno, Sacramento, and San Ramon. We considered daily average temperature missing if fewer than 24 of the 48 possible half-hourly temperatures were reported for that day.

For each station-hour of the NOAA data, we computed an index of climatic severity called the temperature-humidity index (THI). This index was used in developing the time-temperature matrices and in the allocation of annual to peak day energy use. The definition we used to compute THI is:

$$
T H I=0.4 *(\text { dry-bulb temperature }+ \text { wet-bulb temperature })+15,
$$

with dry-bulb and wet-bulb temperatures given in degrees Fahrenheit. For some of our analyses we also used THI degree-days, or THI-DD, as it is used in CEC's Peak 
Demand Forecasting Model to allocate annual demand to peak day demand. We computed THI-DD with a base temperature of 68 degrees:

$$
T H I-D D[\text { day } k]=\sum_{i=1}^{24} \max \{\text { THI }[\text { hour } i \text {, day } k]-68,0\}
$$

THI-DD serves as a measure of those hours that contribute to cooling loads (CEC defines these as hours with THI temperatures in excess of $68^{\circ}$ Fahrenheit).

\section{A.3 PG\&E End-Use Load Data}

Load data for 1985 and 1986 were obtained from PG\&E in 1989 as SAS data sets AMPLBL1 and AMPLBL2. These data sets contained half-hourly loads for both conditioning and non-conditioning appliances and for total household. Cooling load data for 1987, 1988, and 1989 were received from PG\&E in the SAS data sets CECRES87, CECRES88, and LBLRES89. Data for 1987, 1988, and 1989 were reported on an hourly basis. Each data set contained data for total household load in addition to loads for specific appliances.

PG\&E reports load data at the end of the measurement interval. For example, in the 1987,1988 , and 1989 hourly data provided by PG\&E, the 1 a.m. hourly load is the load between 12 midnight and $1 \mathrm{a} . \mathrm{m}$.. We used this convention to compute hourly average loads from the 1985 and 1986 half-hourly load data. For consistency with PG\&E's reporting procedure, we defined the hourly load for a given hour as the average of the loads reported for the two preceding half-hours. For example, load demand for 1 a.m. was computed as the average of the 12:30 a.m. and $1 \mathrm{a}$.m. loads. If data for only one of the two half-hours was available, we estimated the corresponding hourly demand using only the demand for the half-hour.

\section{A.4 Time Conventions}

The NOAA weather data are always reported in Local Standard Time whereas the PG\&E weather and load data are recorded using Daylight Savings Time. We converted the NOAA data to Daylight Savings Time for our computations.

For this report Weekend is defined as Saturday, Sunday, or one of the eight holidays listed on the PG\&E rate schedule: New Year's Day, President's Day, Memorial Day, 
Independence Day, Labor Day, Veteran's Day, Thanksgiving Day, and Christmas Day. A Weekday is any other day. 
Table A-1. Number of Central and Room Air Conditioners Metered by CEC Region and PG\&E Zone

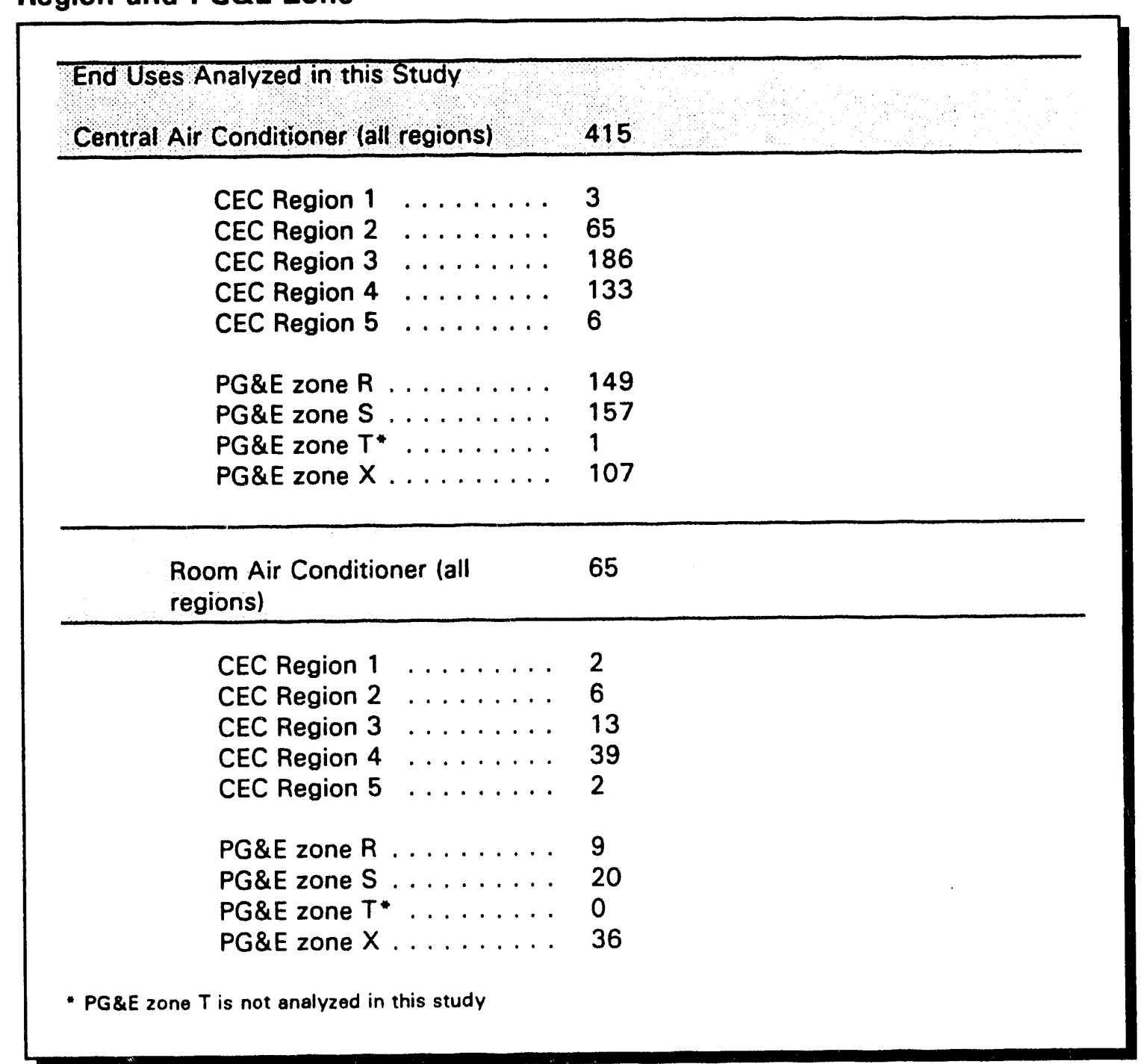


Table A-2. Comparison of Geographic Assignments Between PG\&E Zones and CEC Regions

\begin{tabular}{|c|c|c|c|c|c|}
\hline \multirow[b]{2}{*}{$\begin{array}{l}\text { CEC } \\
\text { Region }\end{array}$} & \multirow[b]{2}{*}{$\mathbf{R}$} & \multirow[b]{2}{*}{$S$} & \multicolumn{2}{|c|}{ PG\&E Zone } & \multirow[b]{2}{*}{ TOTAL } \\
\hline & & & $\mathrm{T}$ & $x$ & \\
\hline $\begin{array}{l}1 \\
2 \\
3 \\
4 \\
5 \\
6 \\
7\end{array}$ & $\begin{array}{l}6 \\
1 \\
150 \\
0 \\
i \\
0 \\
25 \\
183\end{array}$ & $\begin{array}{l}15 \\
83 \\
94 \\
43 \\
0 \\
8 \\
0 \\
243\end{array}$ & $\begin{array}{l}19 \\
0 \\
0 \\
23 \\
40 \\
0 \\
0 \\
82\end{array}$ & $\begin{array}{l}0 \\
1 \\
1 \\
226 \\
39 \\
0 \\
0 \\
267\end{array}$ & $\begin{array}{l}40 \\
85 \\
245 \\
292 \\
80 \\
8 \\
25 \\
775\end{array}$ \\
\hline $\begin{array}{l}1 \text { all AMP } \\
\text { metered. }\end{array}$ & & 5 cross & & r which & d uses were \\
\hline
\end{tabular}


Spring Weekday load profile for Zone R

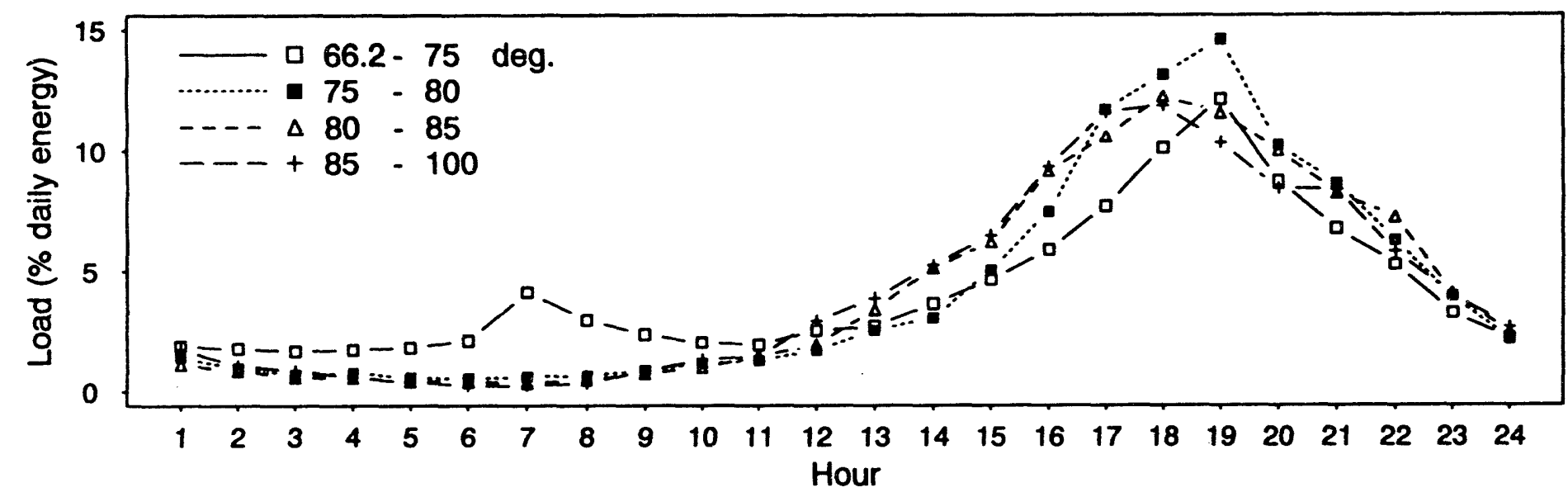

\section{Spring Weekend load profile for Zone R}

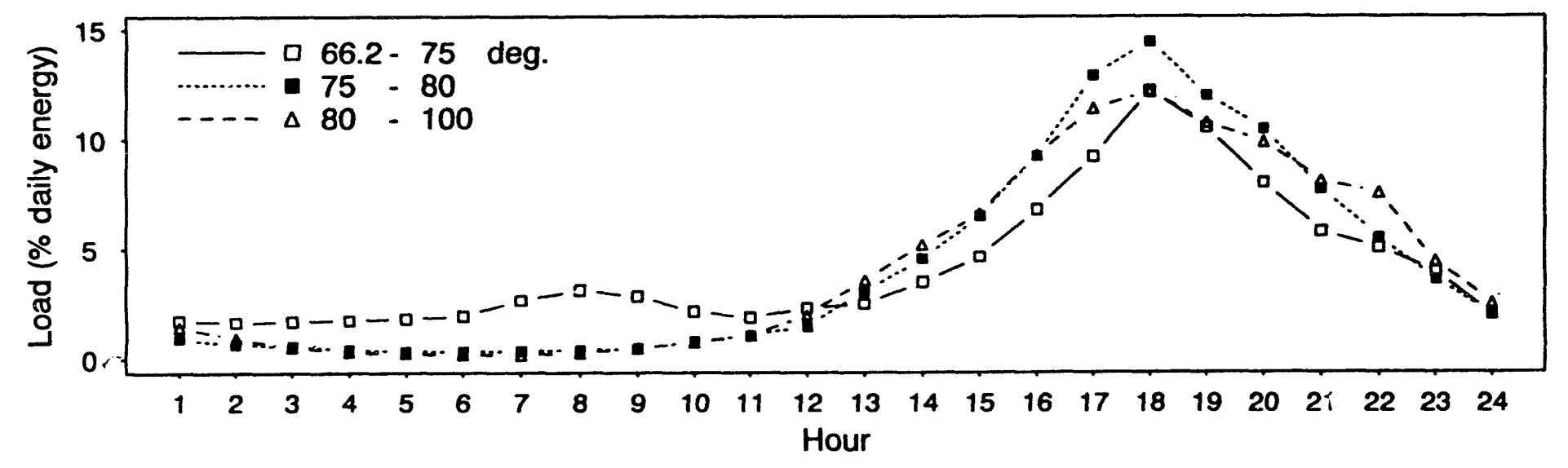

Figure B-1. Central Air Conditioner Load Profiles by Bin for PG\&E Zone R. 
Summer Weekday load profile for Zone R

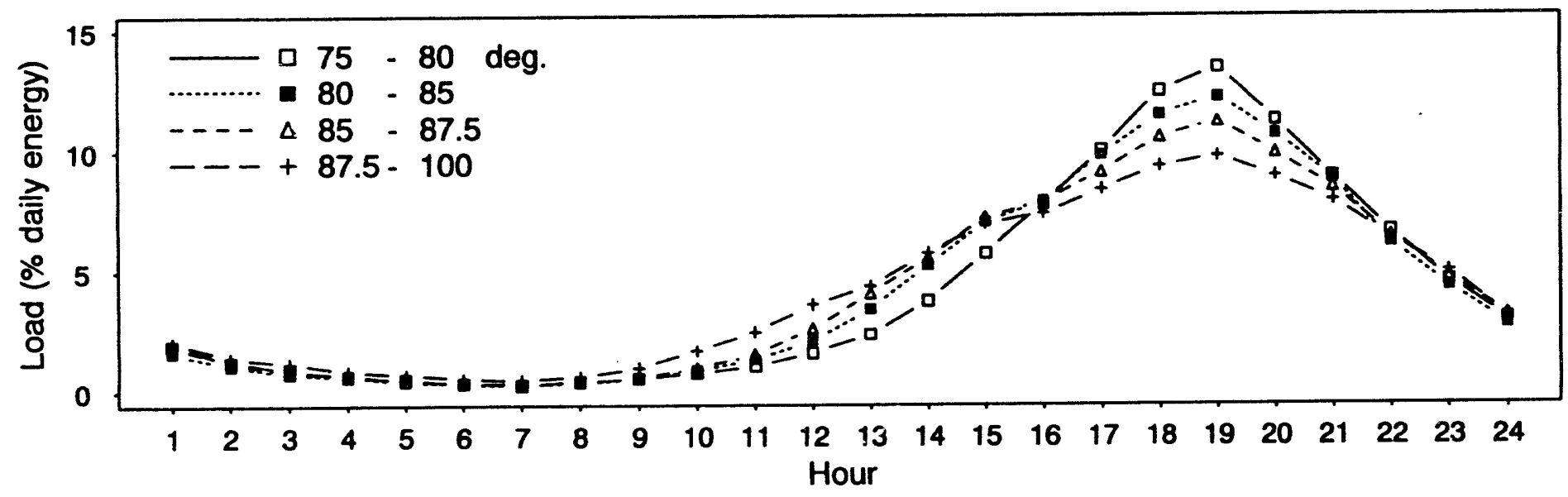

Summer Weekend load profile for Zone R

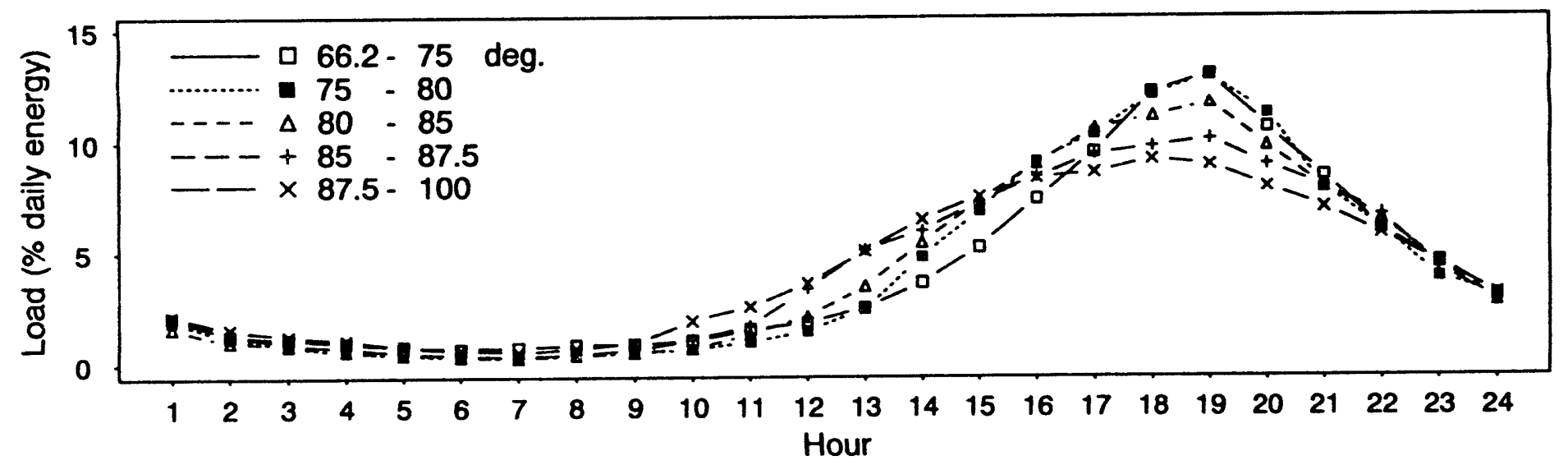

FigureB -2. Central Air Conditioner Load Profiles by Bin for PG\&E Zone R. 
Fall Weekday load profile for Zone R

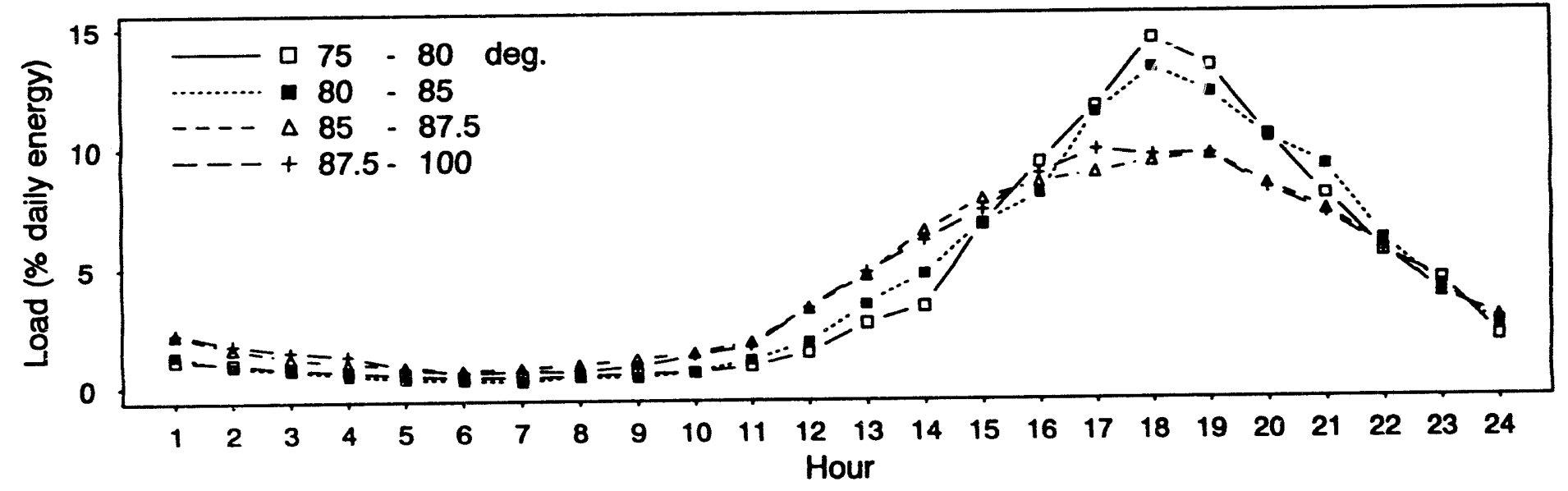

Fall Weekend load profile for Zone $\mathbf{R}$

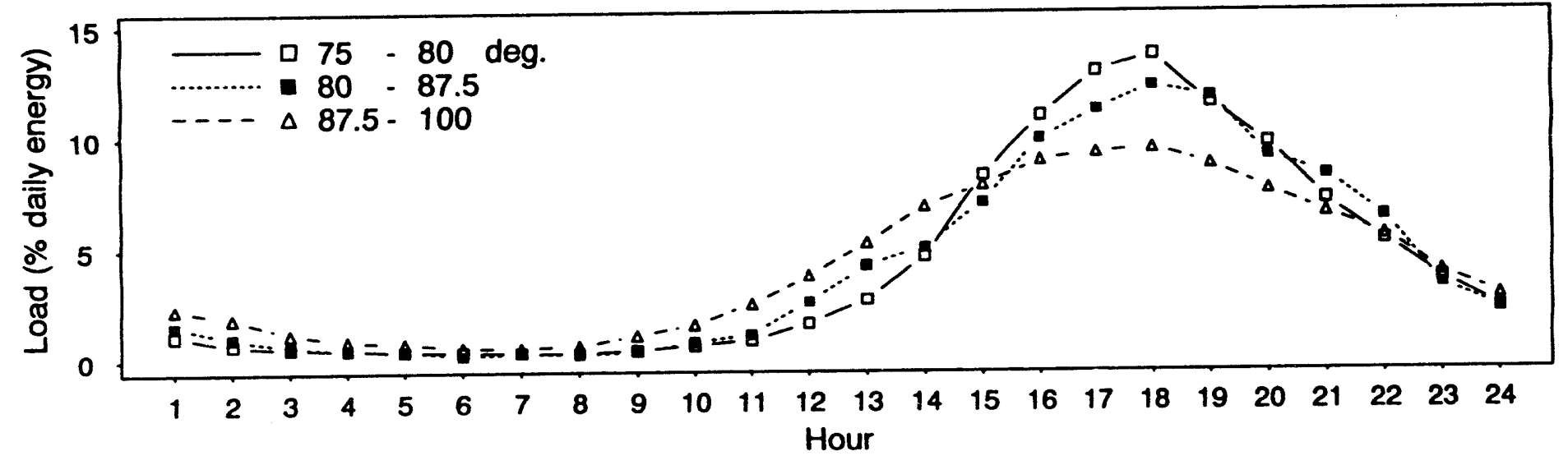

Figure B-3. Central Air Conditioner Load Profiles by Bin for PG\&E Zone R. 


\section{Spring Weekday load profile for Zone S}

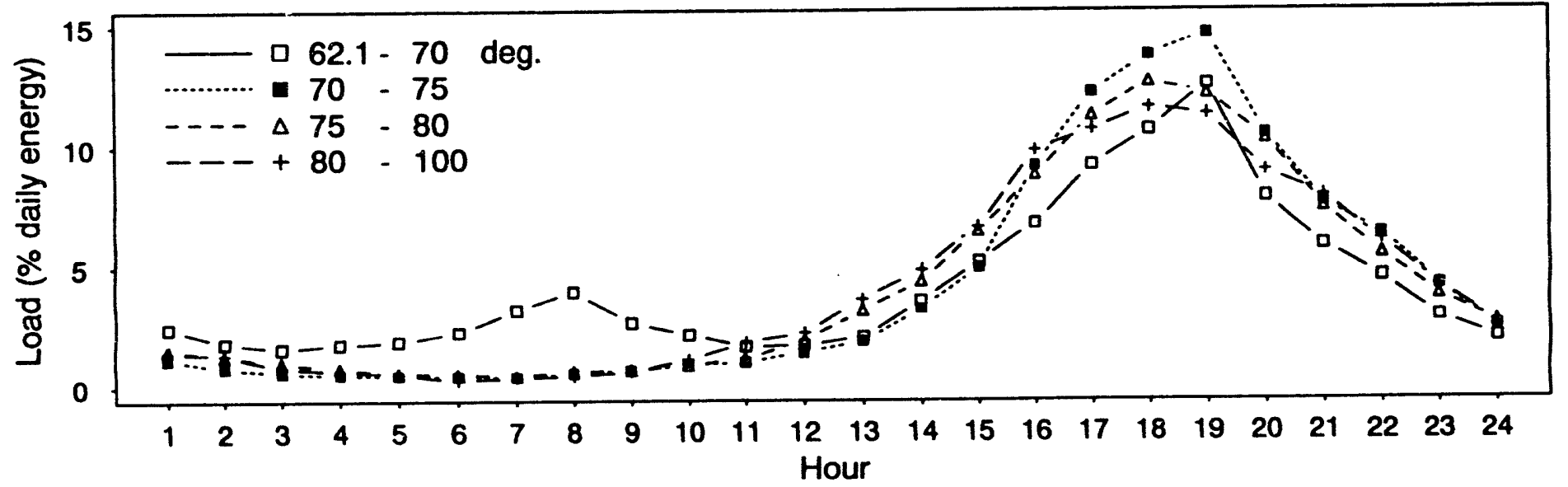

\section{Spring Weekend load profile for Zone S}

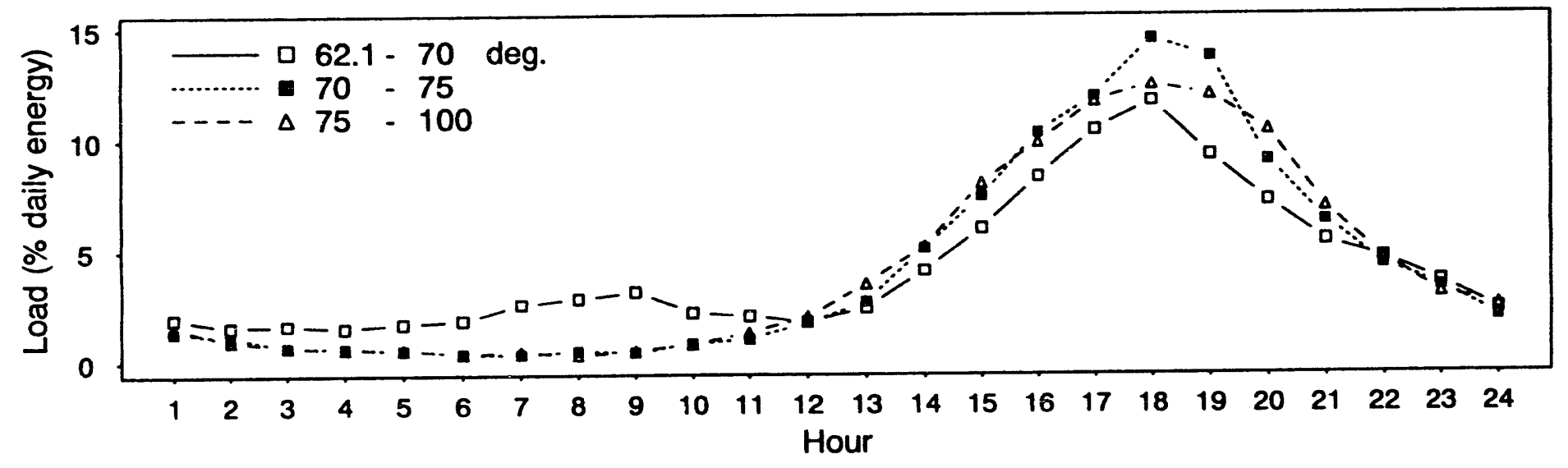

Figure B -4. Central Air Conditioner Load Profiles by Bin for PG\&E Zone S. 


\section{Summer Weekday load profile for Zone S}

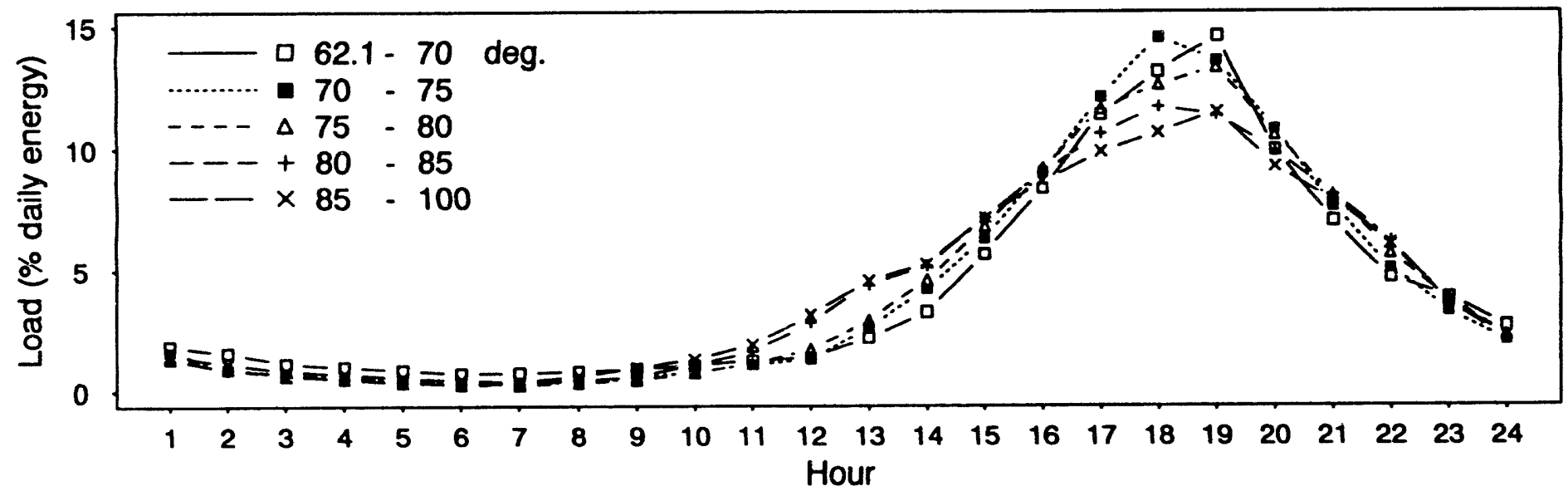

Summer Weekend load profile for Zone S

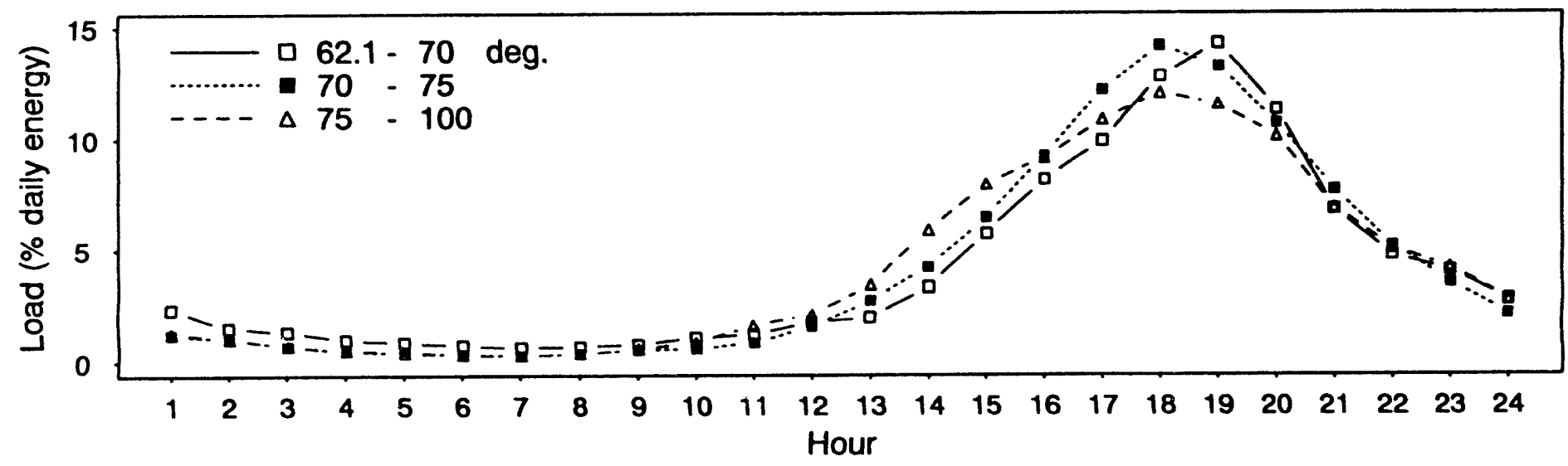

Figure B-5. Central Air Conditioner Load Profiles by Bin for PG\&E Zone S. 
Fall Weekday load profile for Zone S

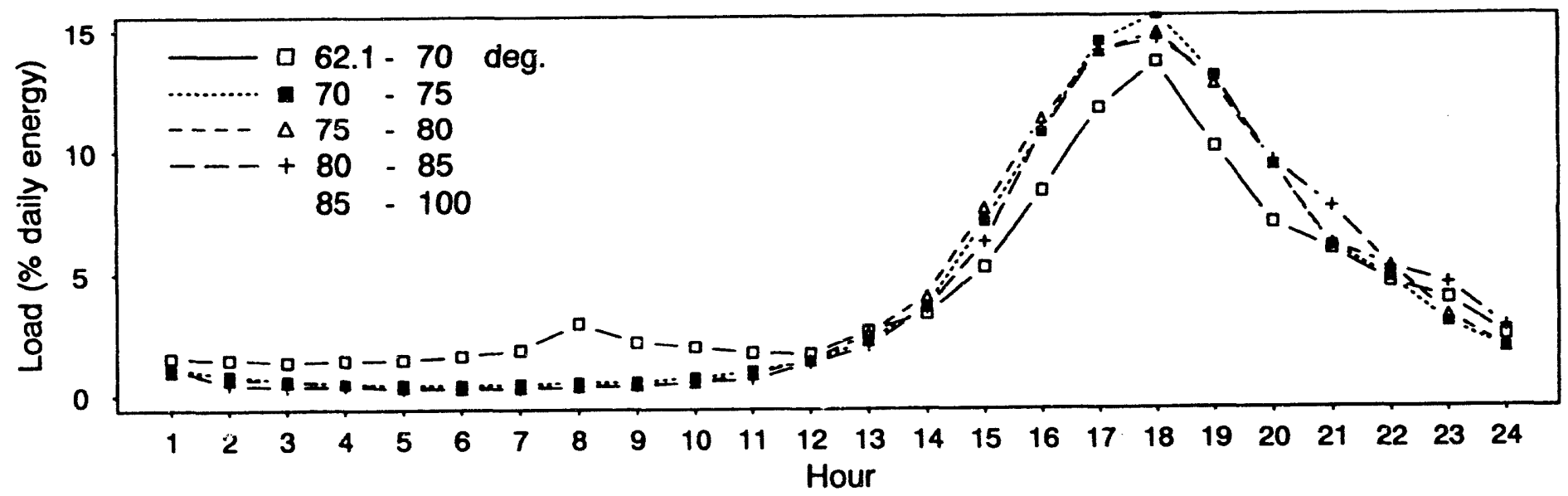

Fall Weekend load profile for Zone S

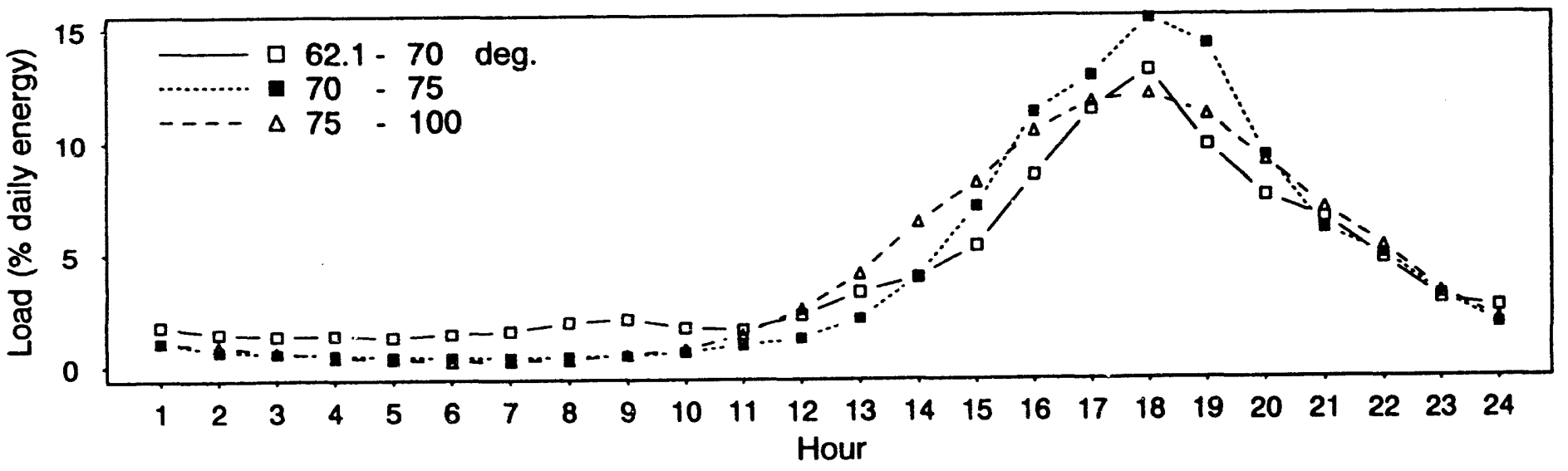

Figure B-6. Central Air Conditioner Load Profiles by Bin for PGí.E Zone S. 


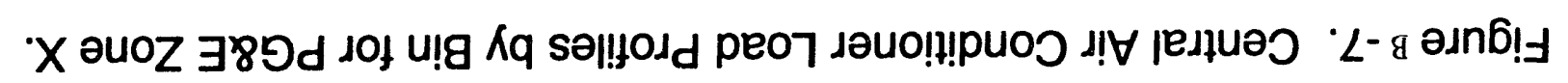

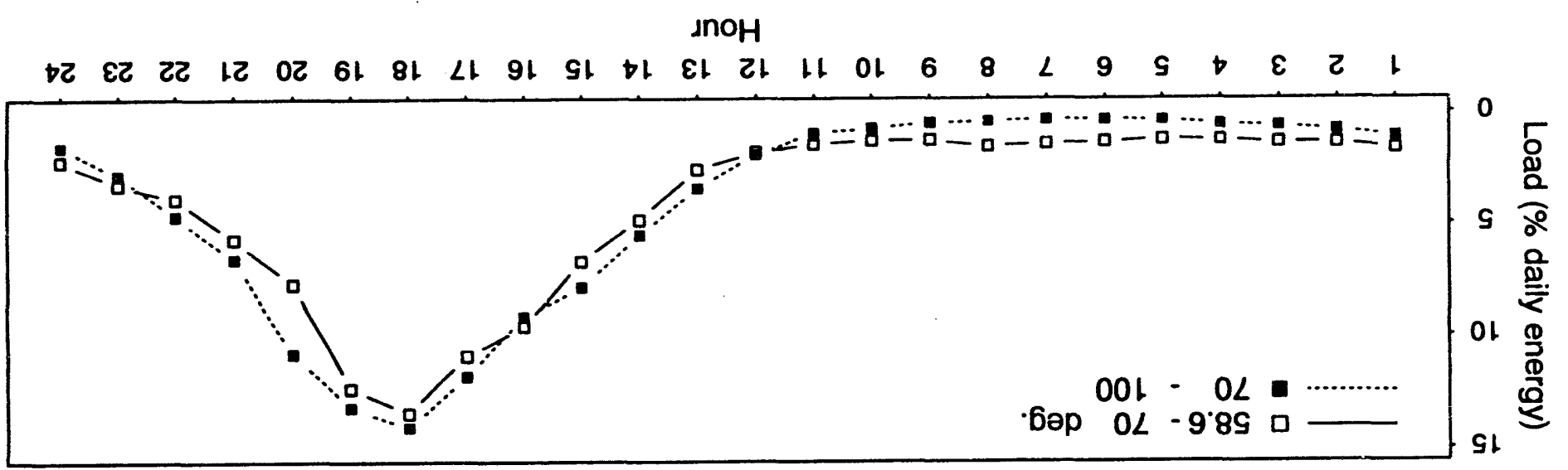

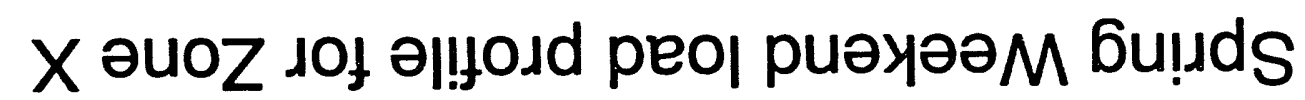

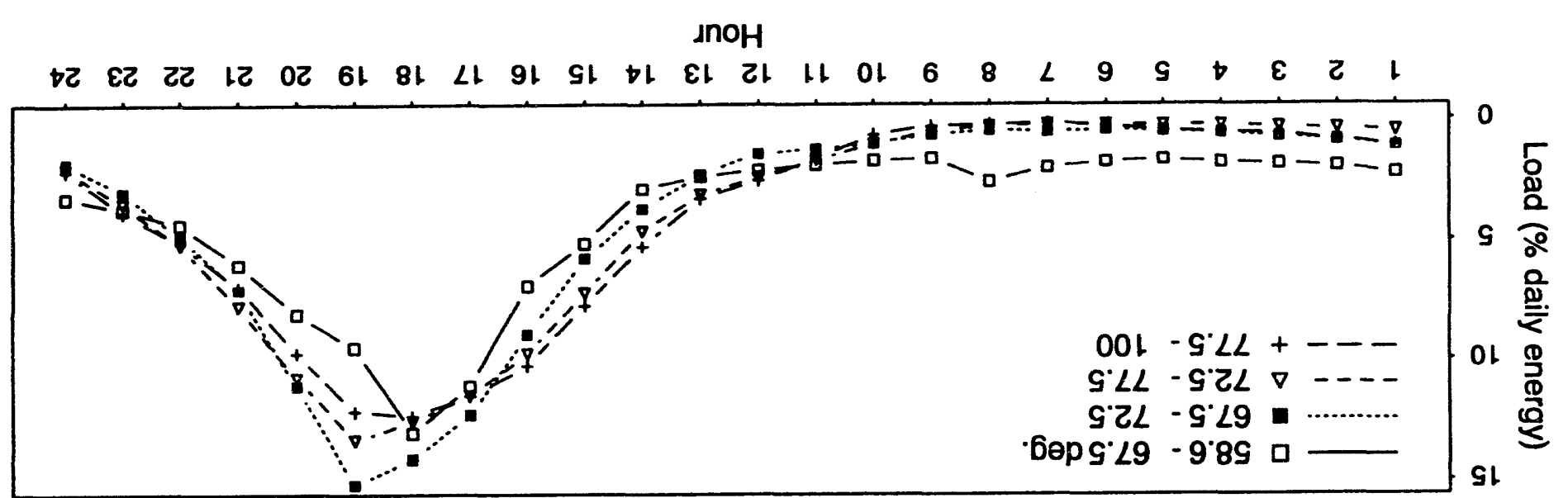

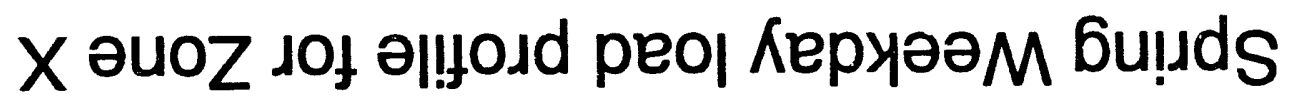




\section{Summer Weekday load profile for Zone X}

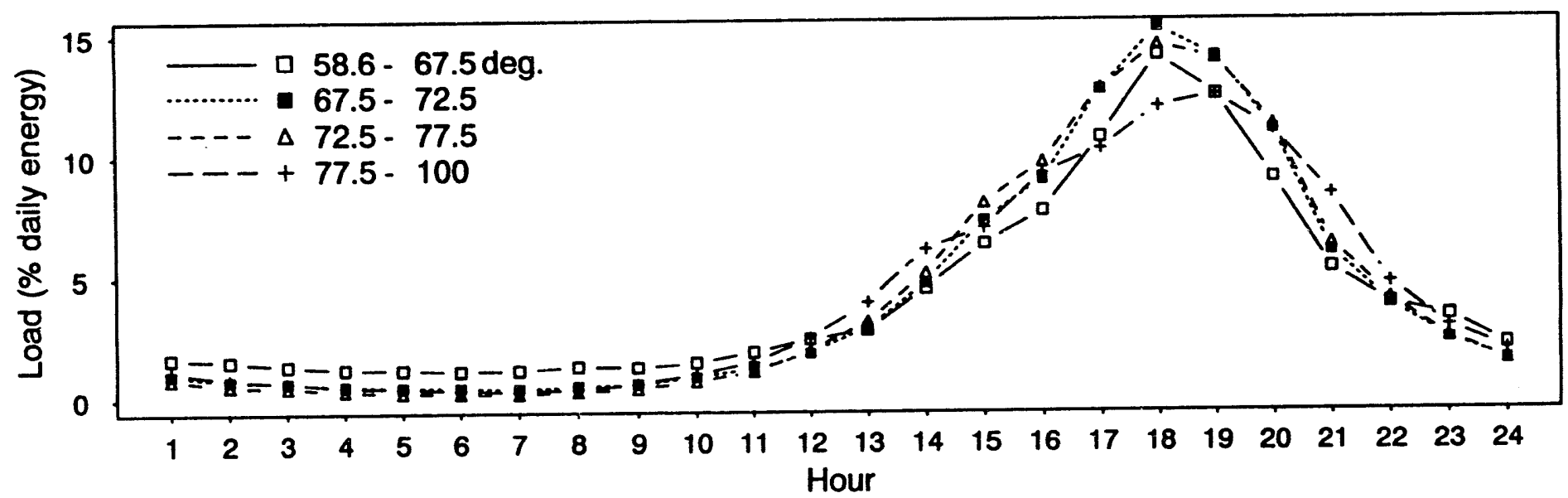

\section{Summer Weekend load profile for Zone X}

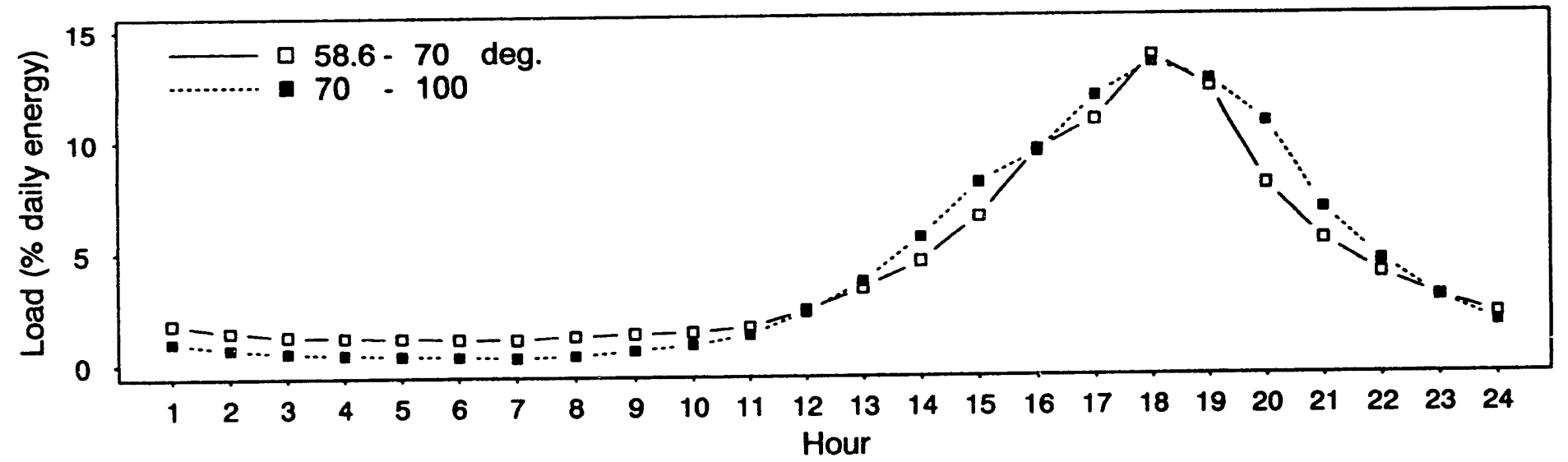

Figure B -8. Central Air Conditioner Load Profiles by Bin for PG\&E Zone X. 
Fall Weekday load profile for Zone X

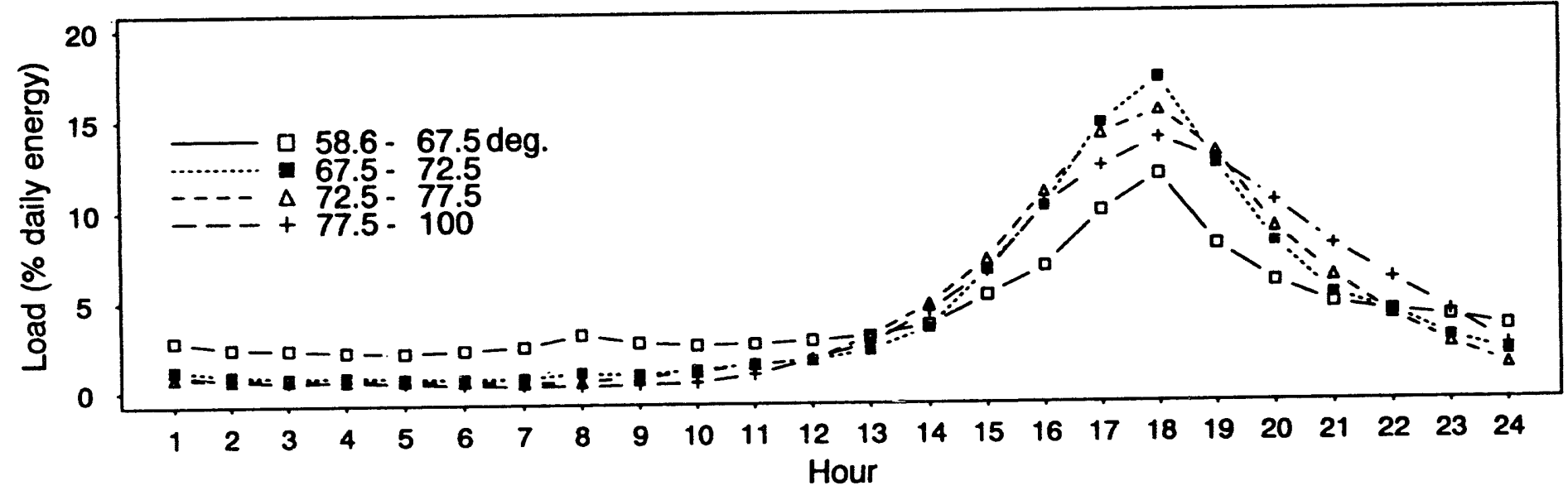

Fall Weekend load profile for Zone X

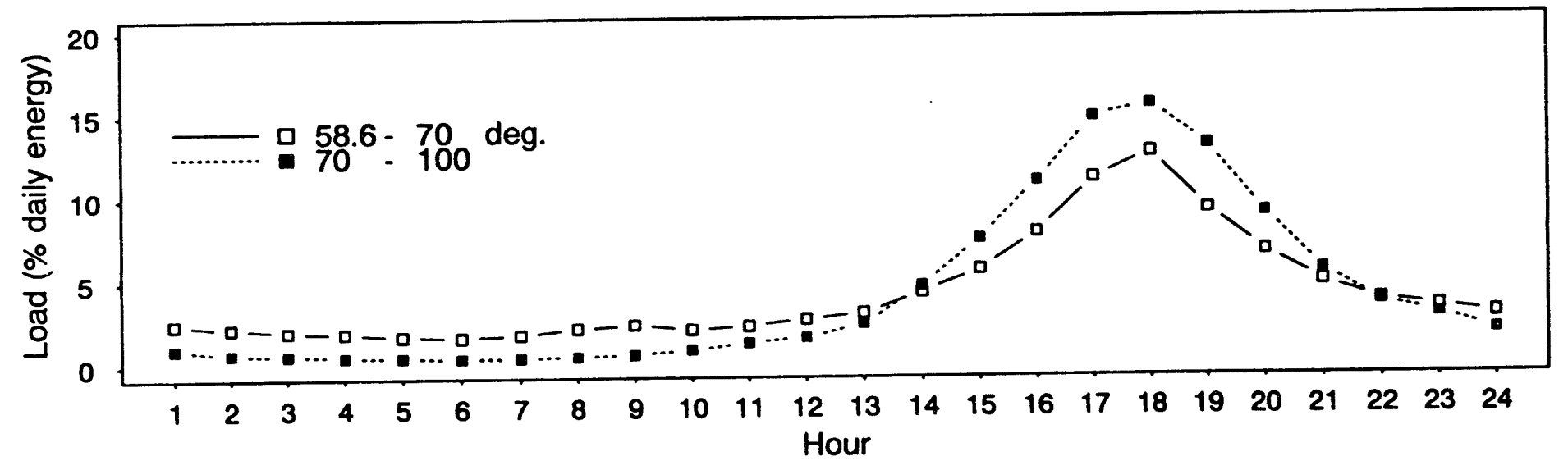

Figure B-9. Central Air Conditioner Load Profiles by Bin for PG\&E Zone X. 


\section{Spring Weekday load profile for Region 2}

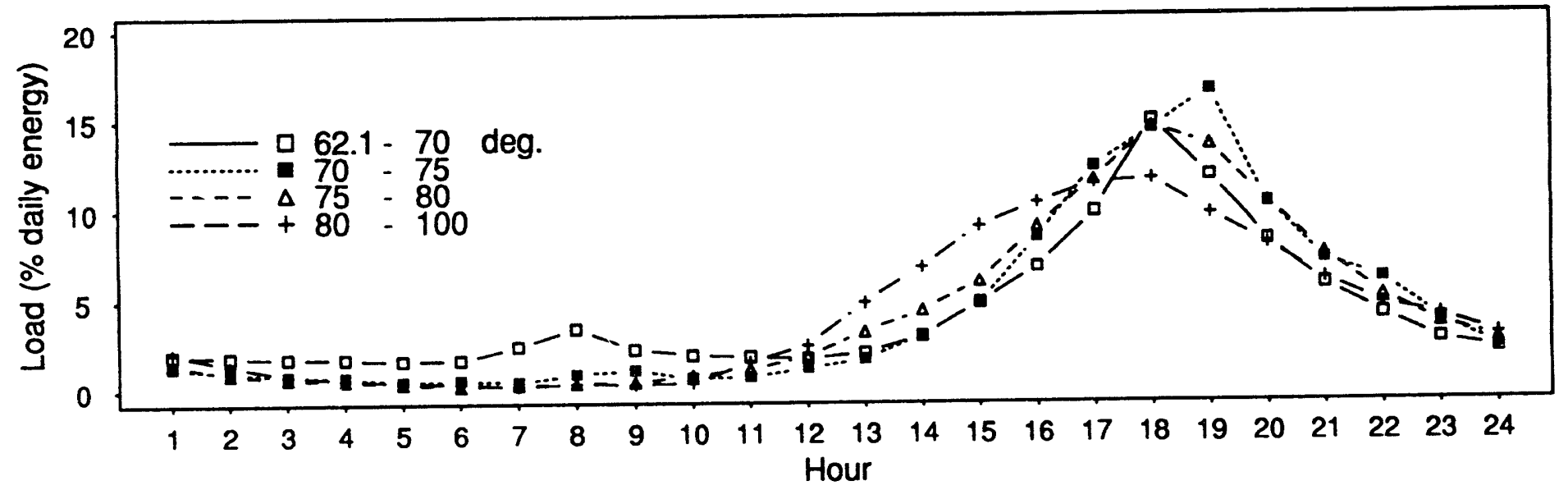

\section{Spring Weekend load profile for Region 2}

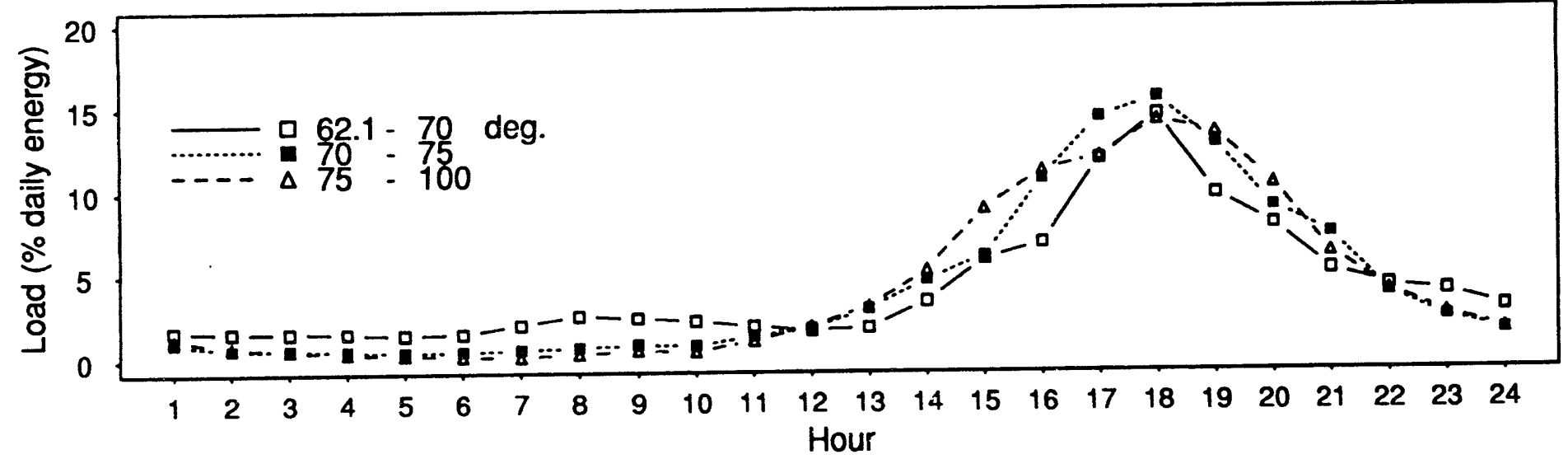

Figure B-10. Central Air Conditioner Load Profiles by Bin for CEC Region 2. 


\section{Summer Weekday load profile for Region 2}

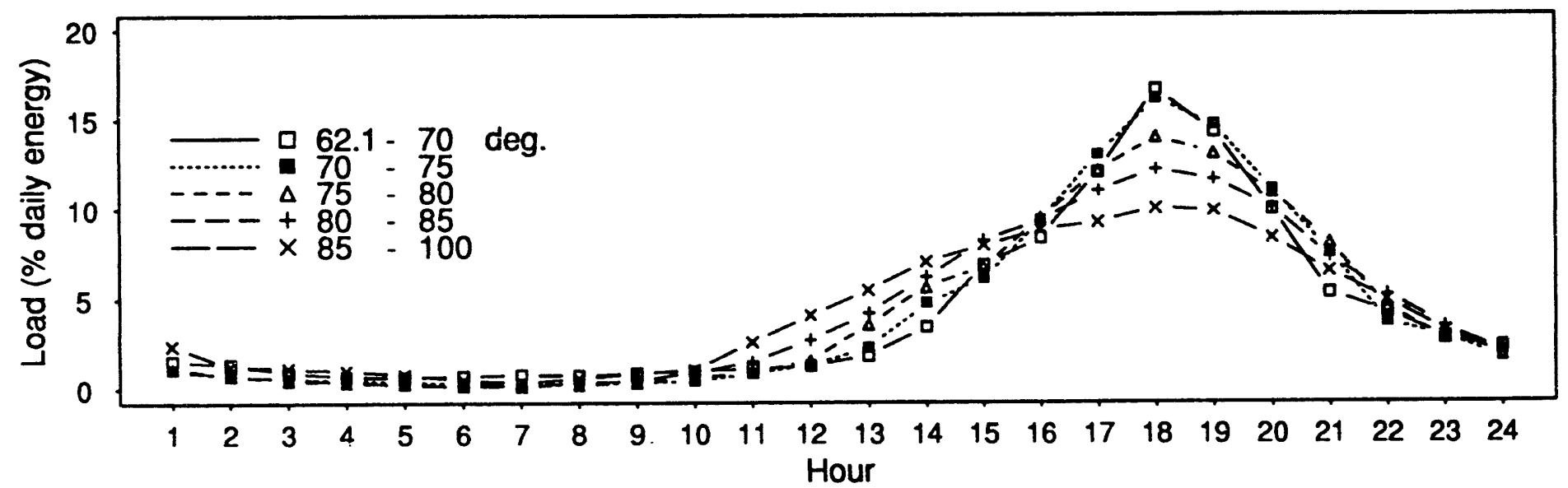

Summer Weekend load profile for Region 2

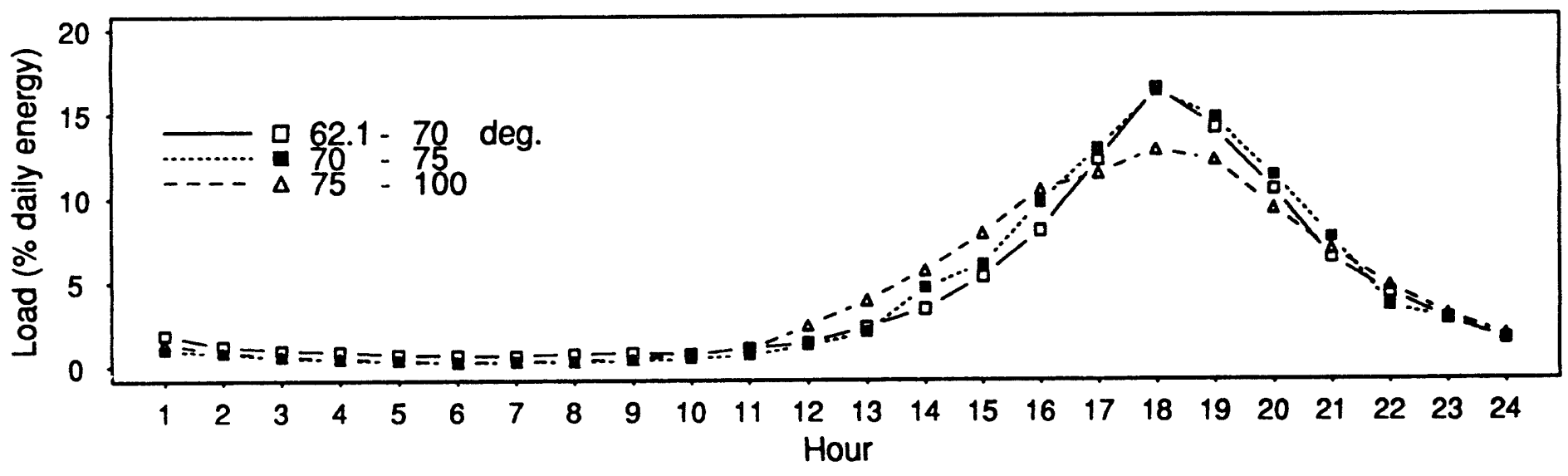

Figure B-11. Central Air Conditioner Load Profiles by Bin for CEC Region 2. 
Fall Weekday load profile for Region 2

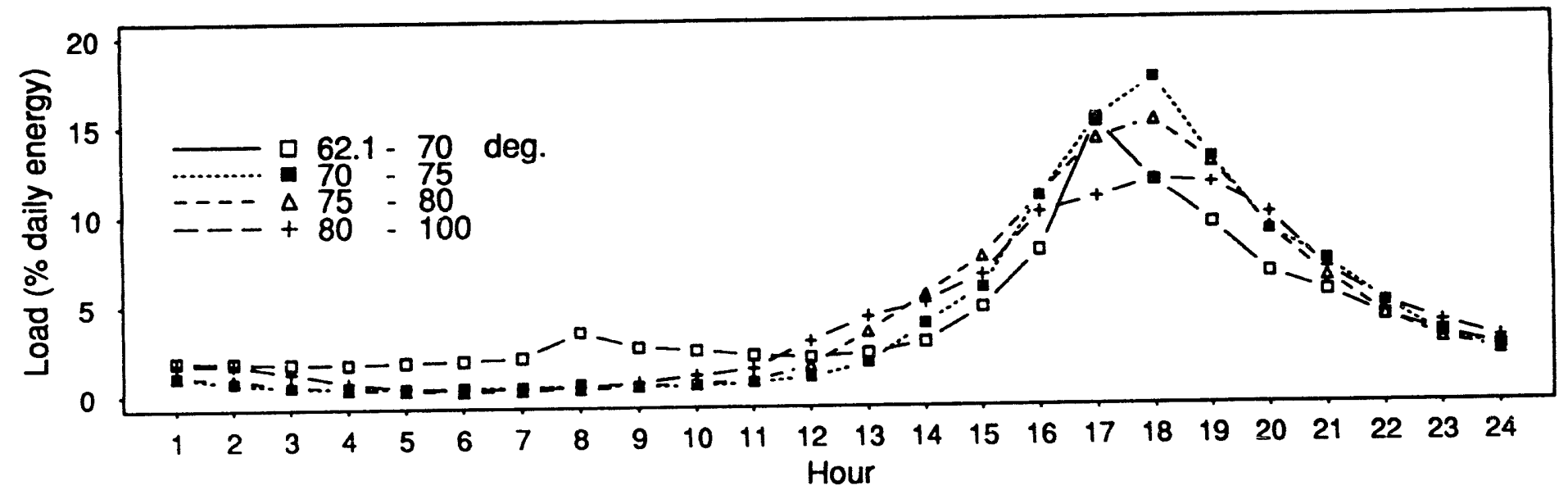

Fall Weekend load profile for Region 2

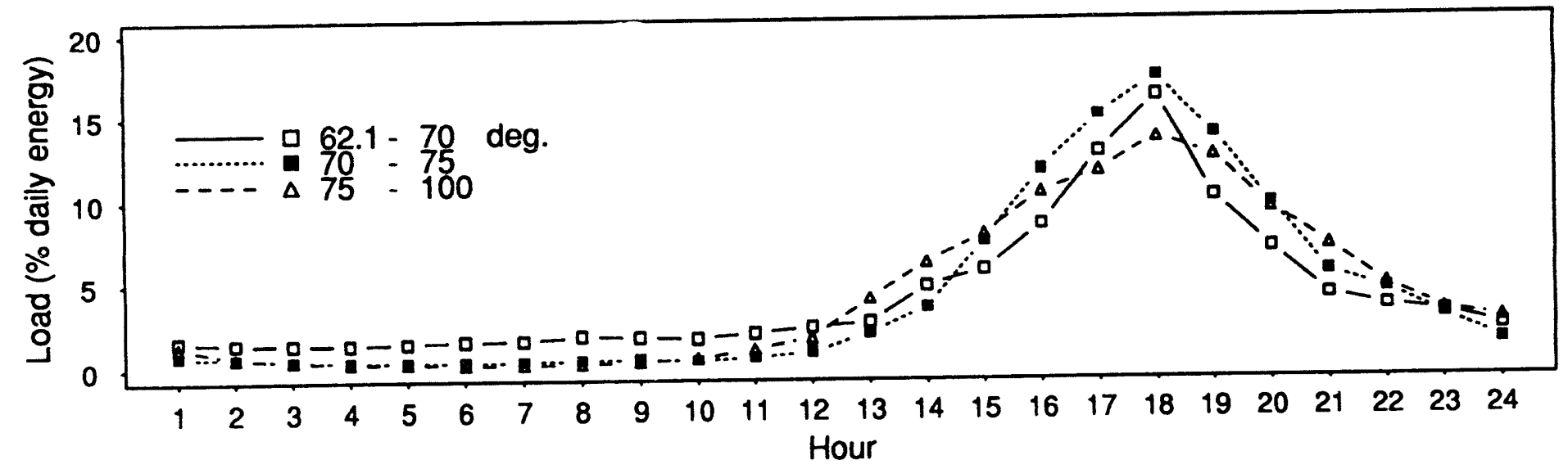

Figure B-12. Central Air Conditioner Load Profiles by Bin for CEC Region 2. 


\section{Spring Weekday load profile for Region 3}

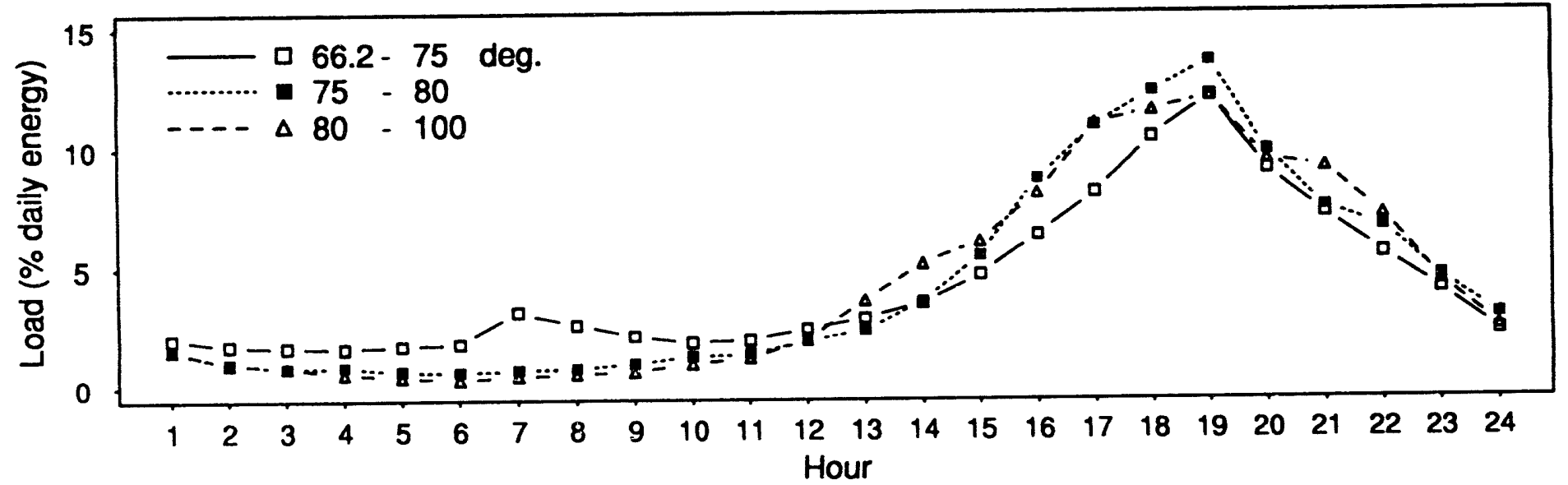

Spring Weekend load profile for Region 3

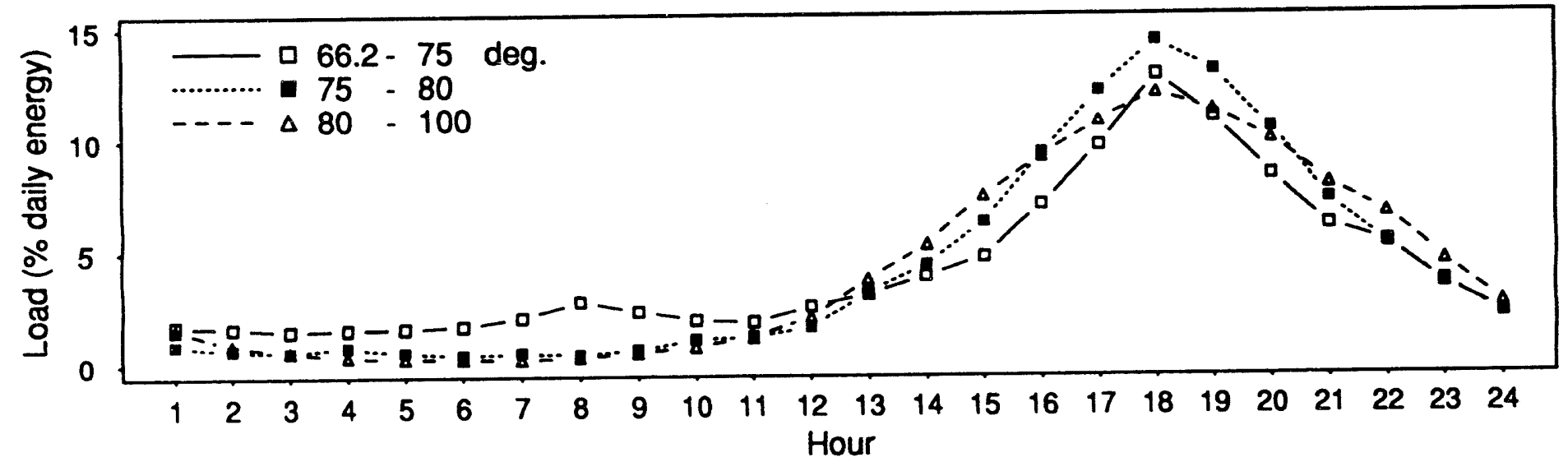

Figure B-13. Central Air Conditioner Load Profiles by Bin for CEC Region 3. 
Summer Weekday load profile for Region 3

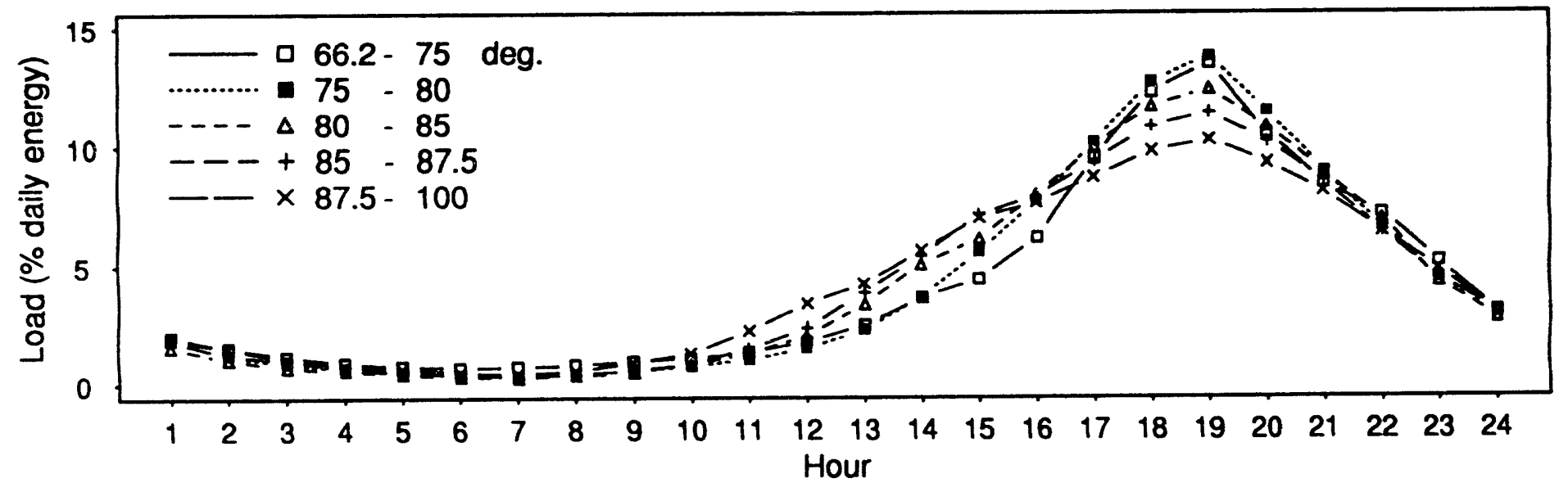

Summer Weekend load profile for Region 3

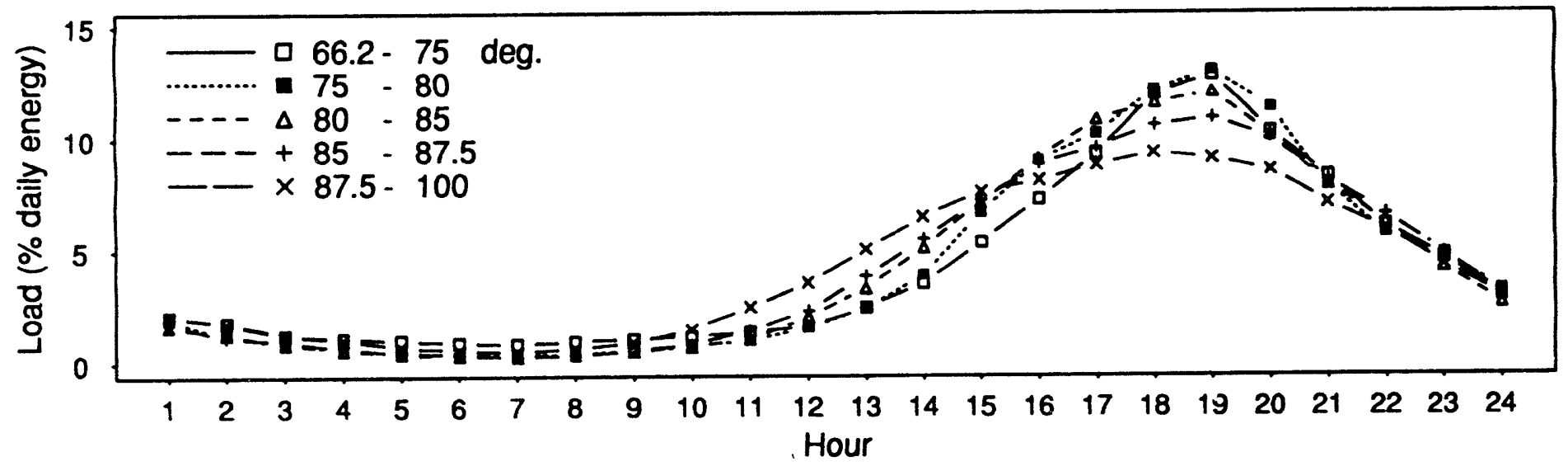

Figure B -14. Central Air Conditioner Load Profiles by Bin for CEC Region 3. 
Fall Weekday load profile for Region 3

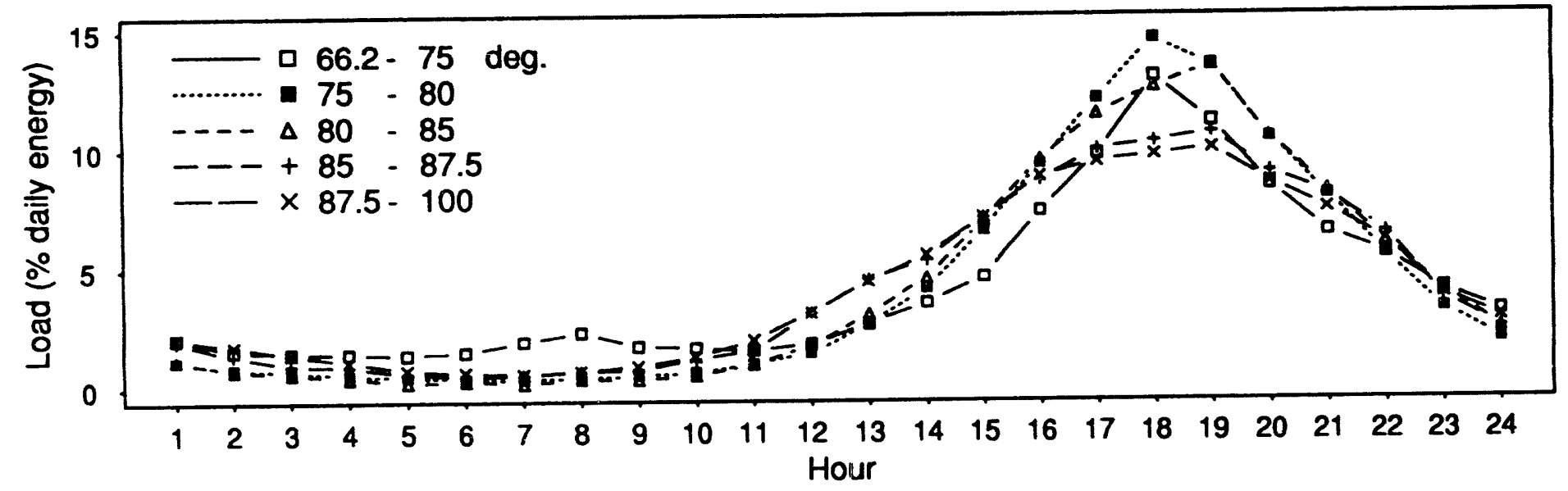

Fall Weekend load profile for Region 3

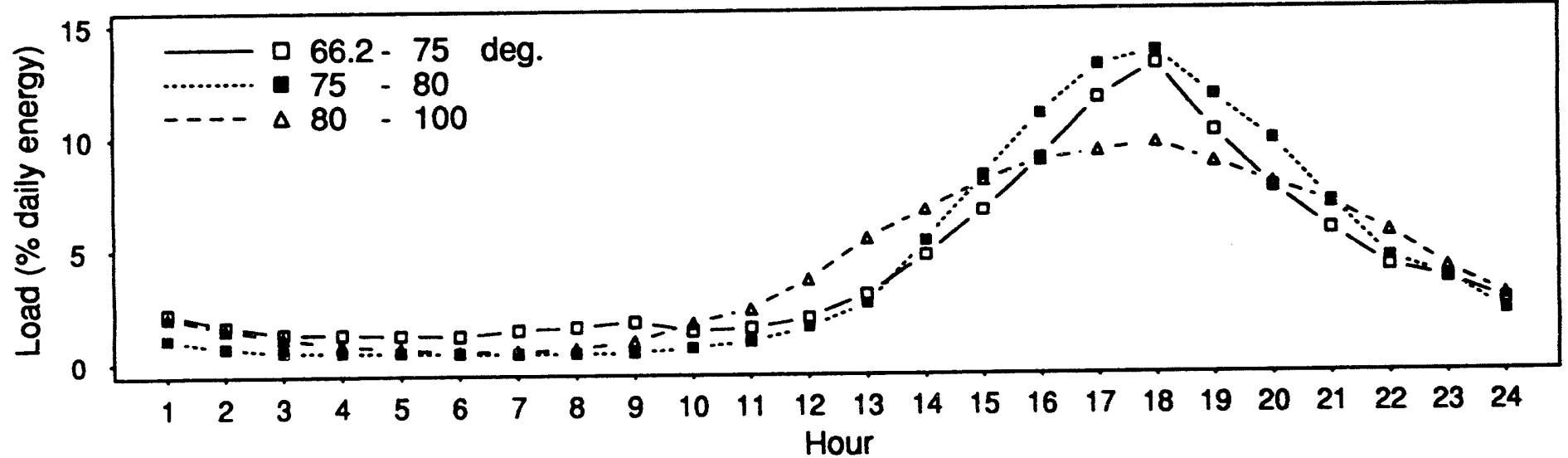

Figure B-15. Central Air Conditioner Load Profiles by Bin for CEC Region 3. 
Spring Weekday load profile for Region 4

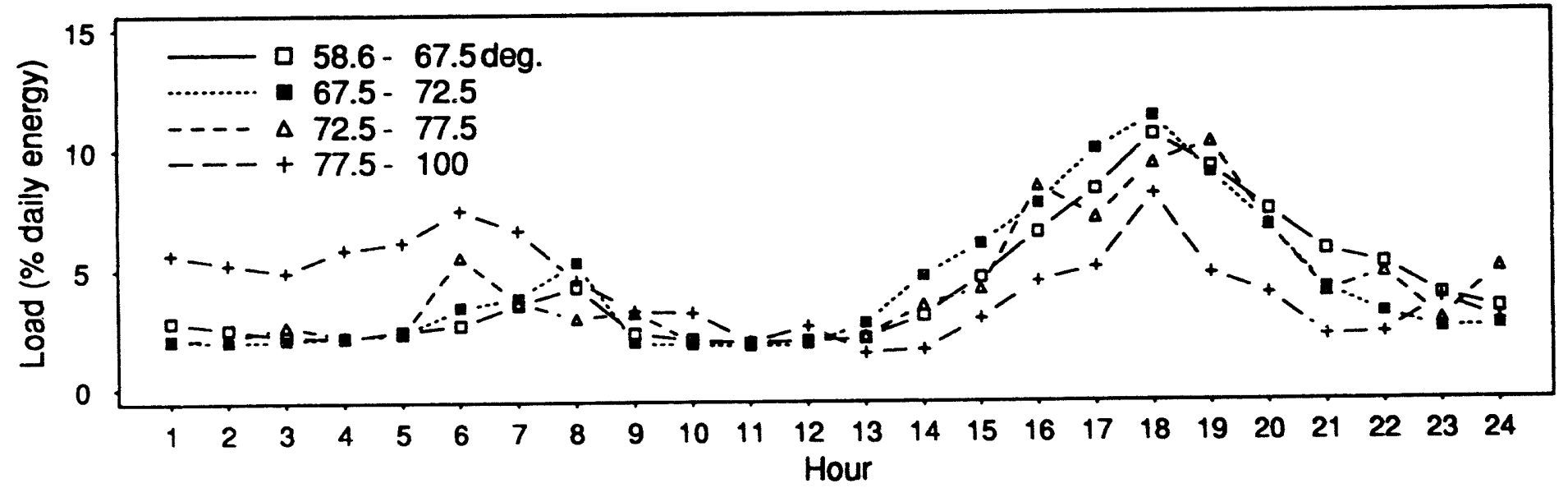

Spring Weekend load profile for Region 4

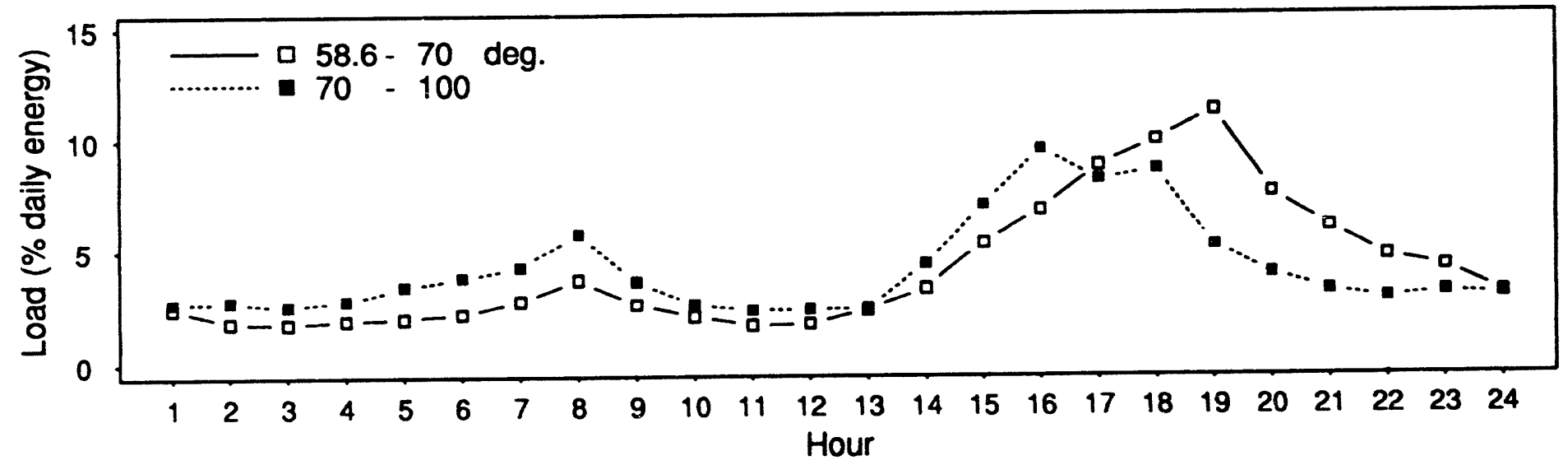

Figure B-16. Central Air Conditioner Load Profiles by Bin for CEC Region 4. 
Summer Weekday load profile for Region 4

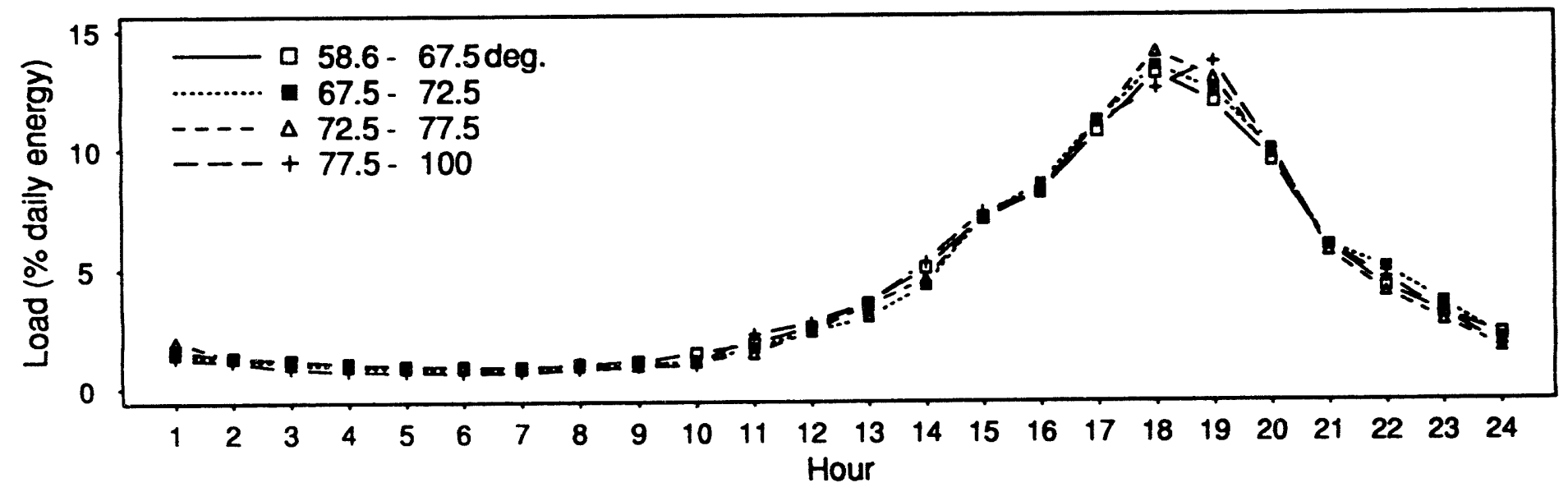

Summer Weekend load profile for Region 4

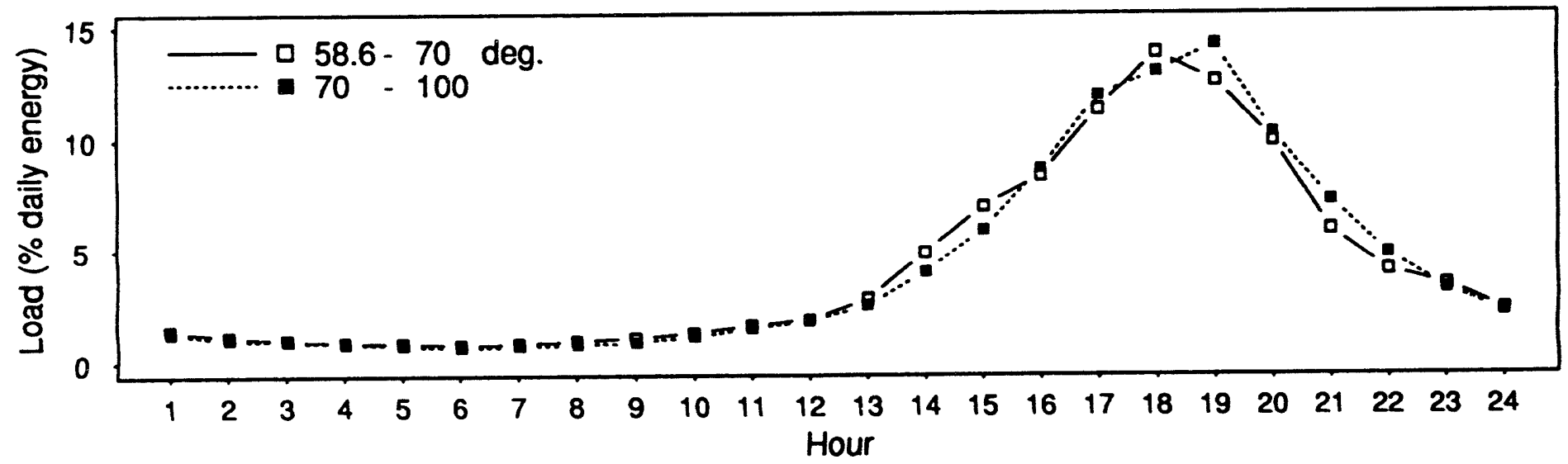

Figure B-17. Central Air Conditioner Load Profiles by Bin for CEC Region 4. 
Fall Weekday load profile for Region 4

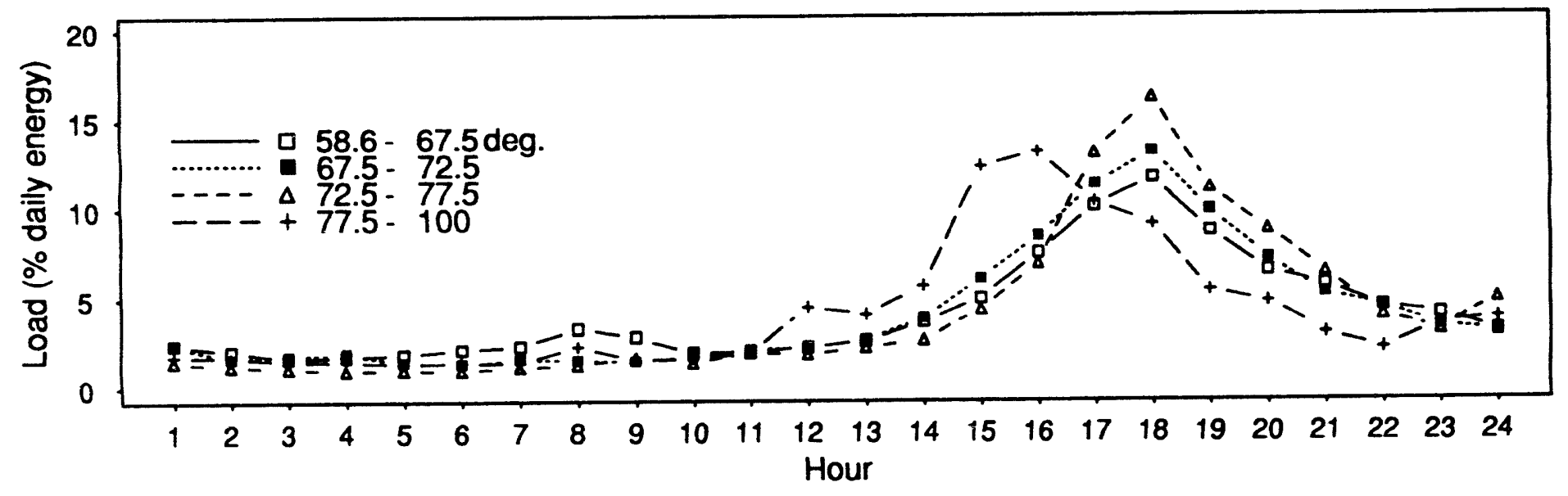

Fall Weekend load profile for Region 4

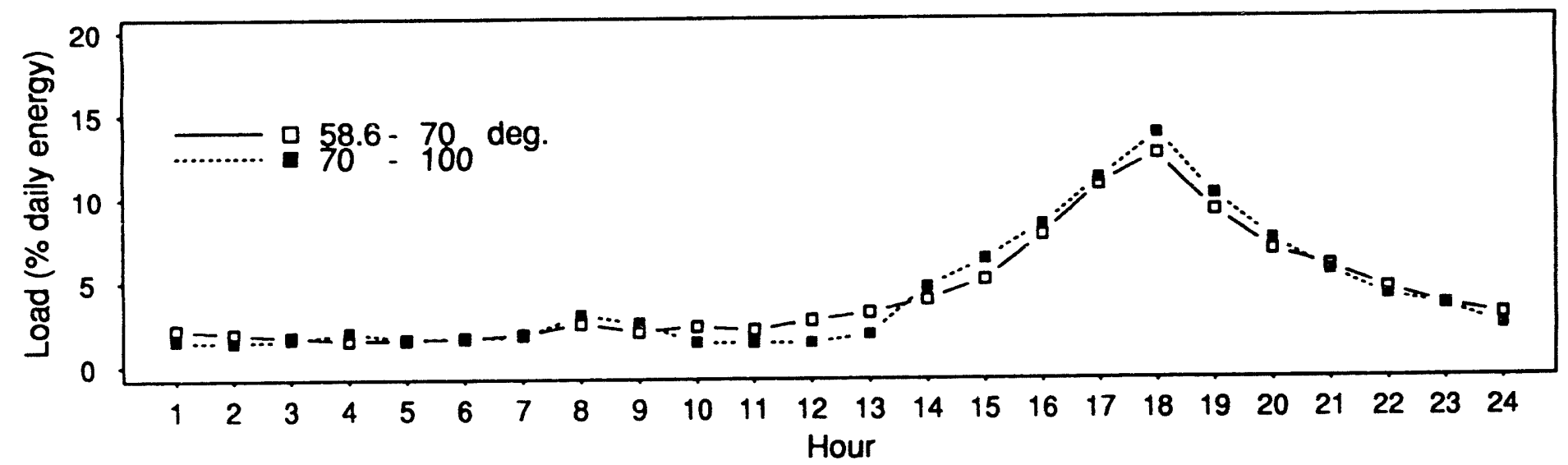

Figure B-18. Central Air Conditioner Load Profiles by Bin for CEC Region 4. 
Appendix C

Plots of Additional Model

Comparisons from Chapter 6 

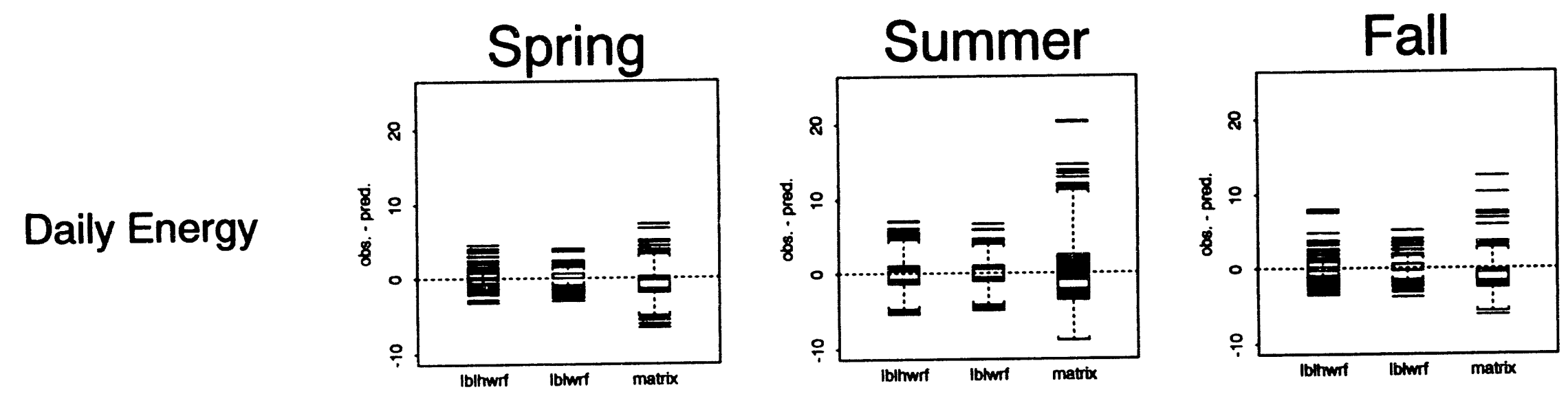

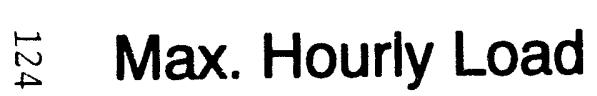
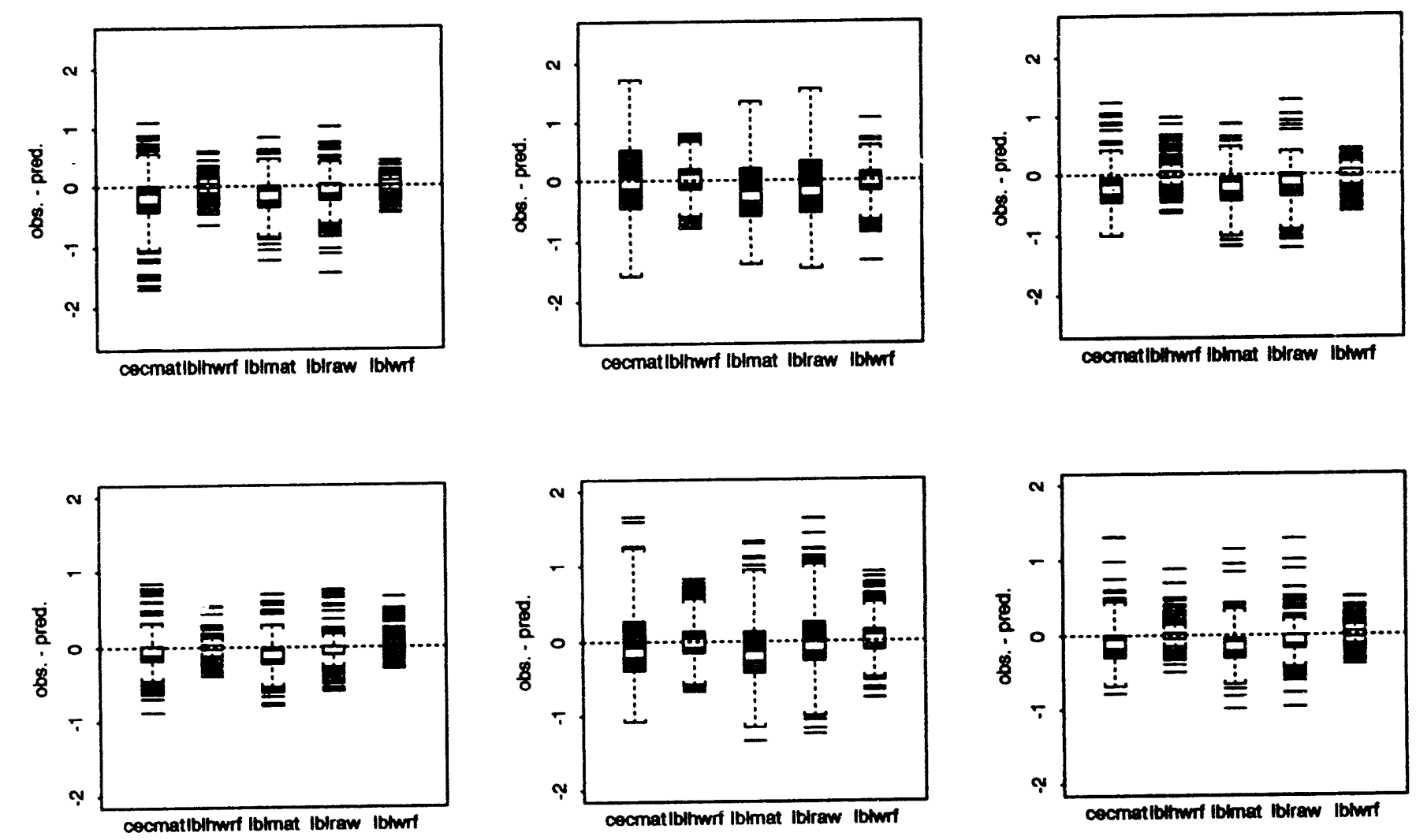

Figure C-1. Distribution of season-specific model residuals (obs. - pred.) for CEC Region 2. 

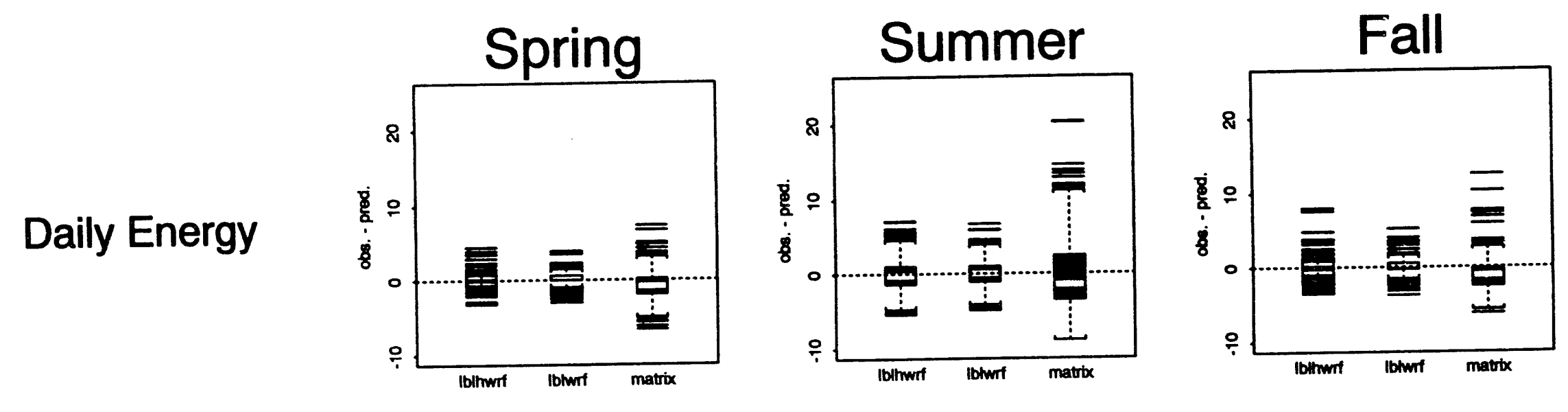

芯 Max. Hourly Load
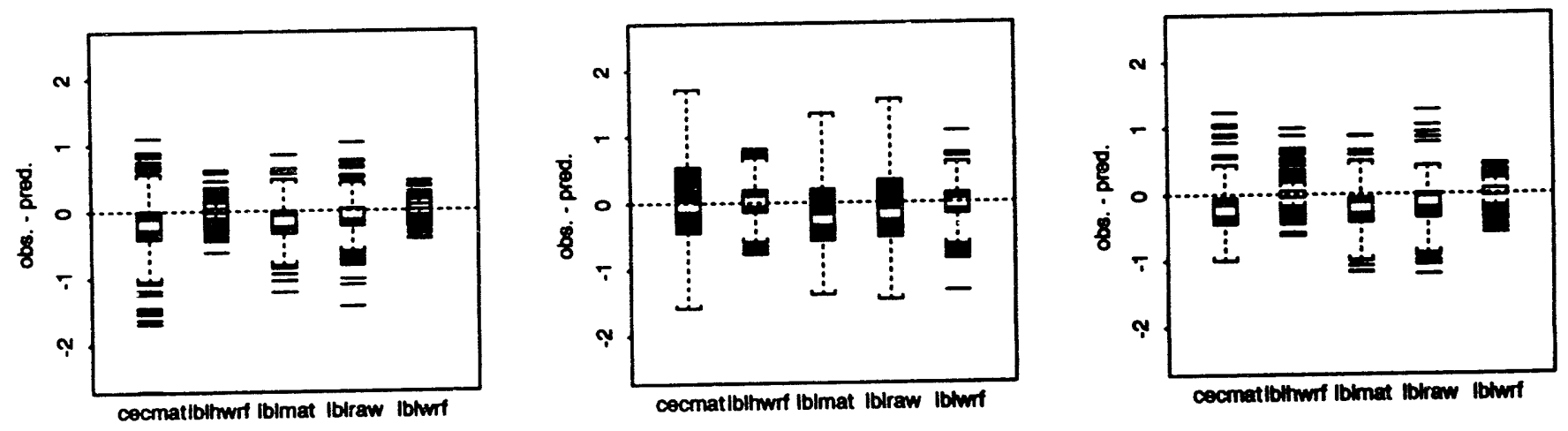

4 p.m. Load
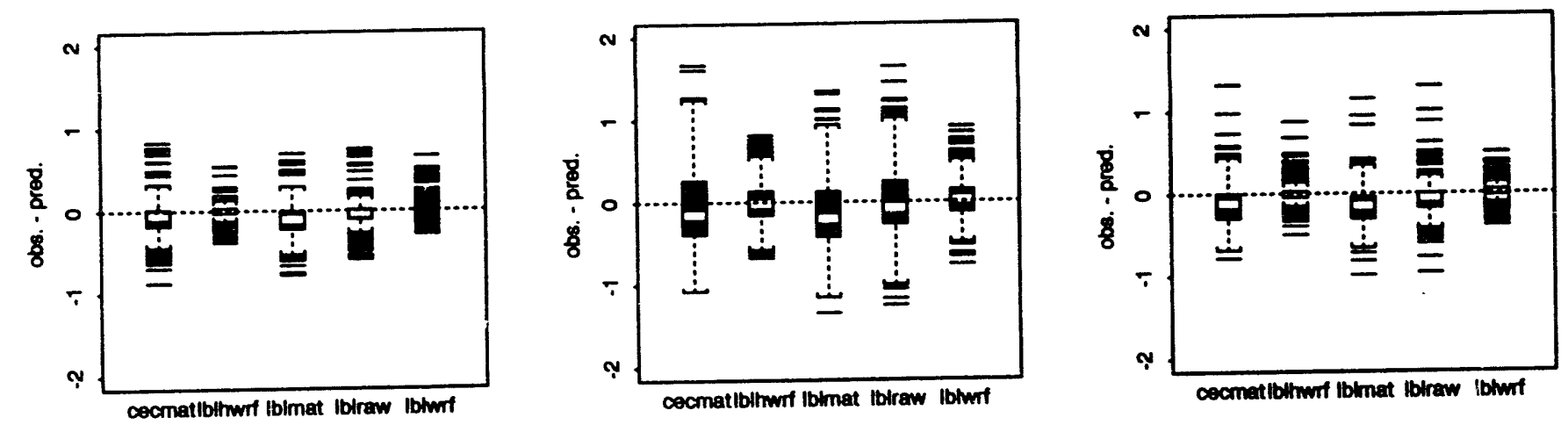

Figure C-2 . Distribution of season-specific model residuals (obs. - pred.) for CEC Region 3 . 

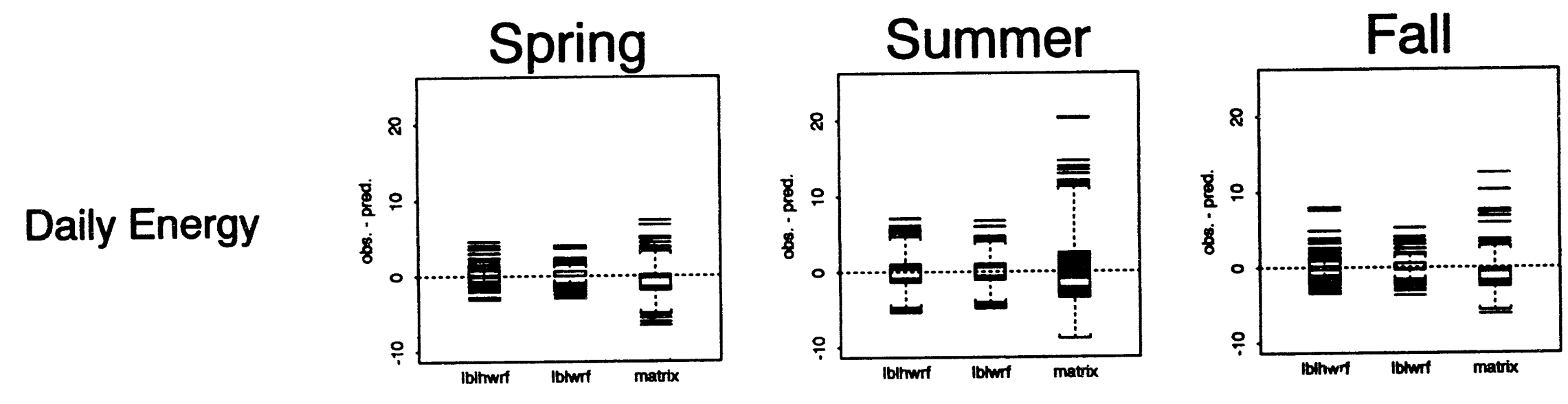

స్ Max. Hourly Load
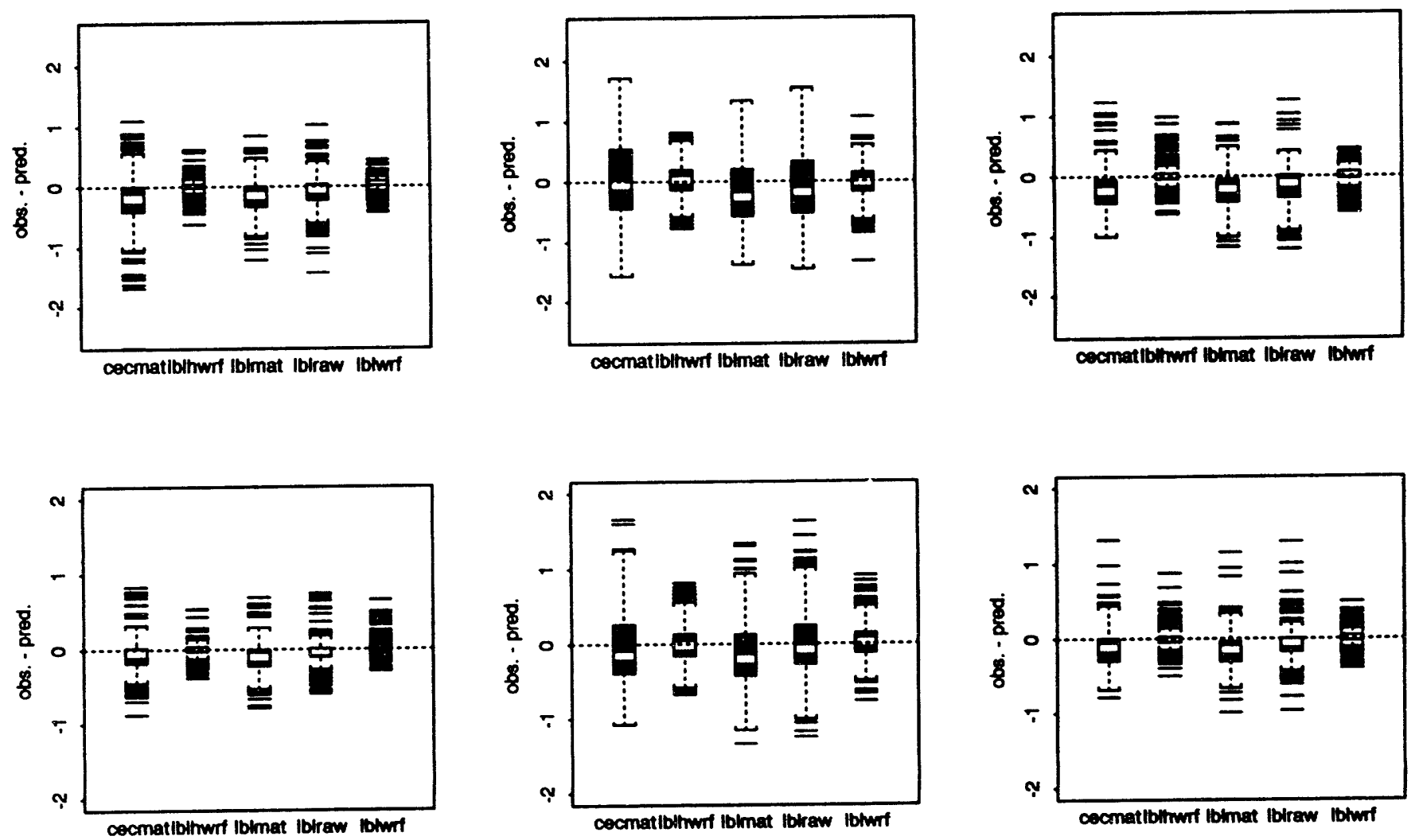

Figure C-3 . Distribution of season-specific model residuals (obs. - pred.) for CEC Region 4. 

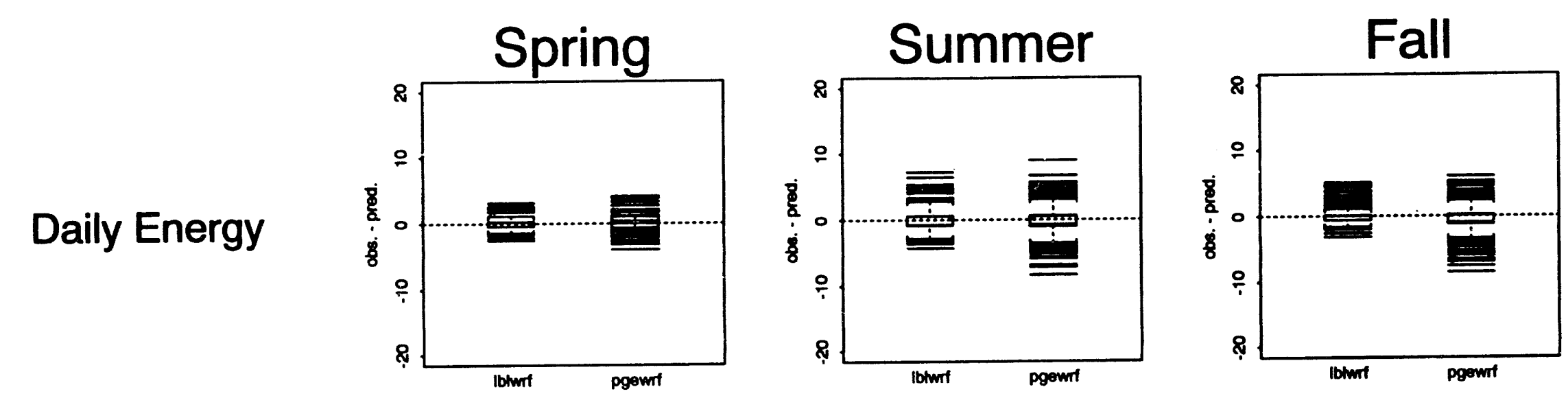

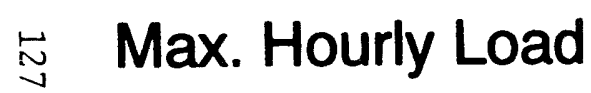
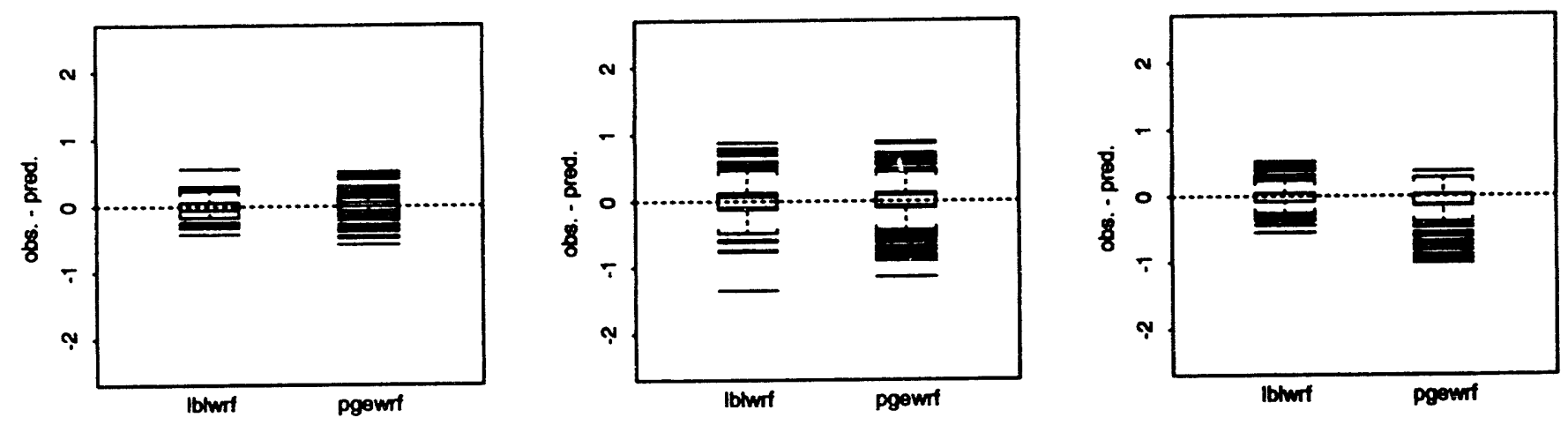

4 p.m. Load
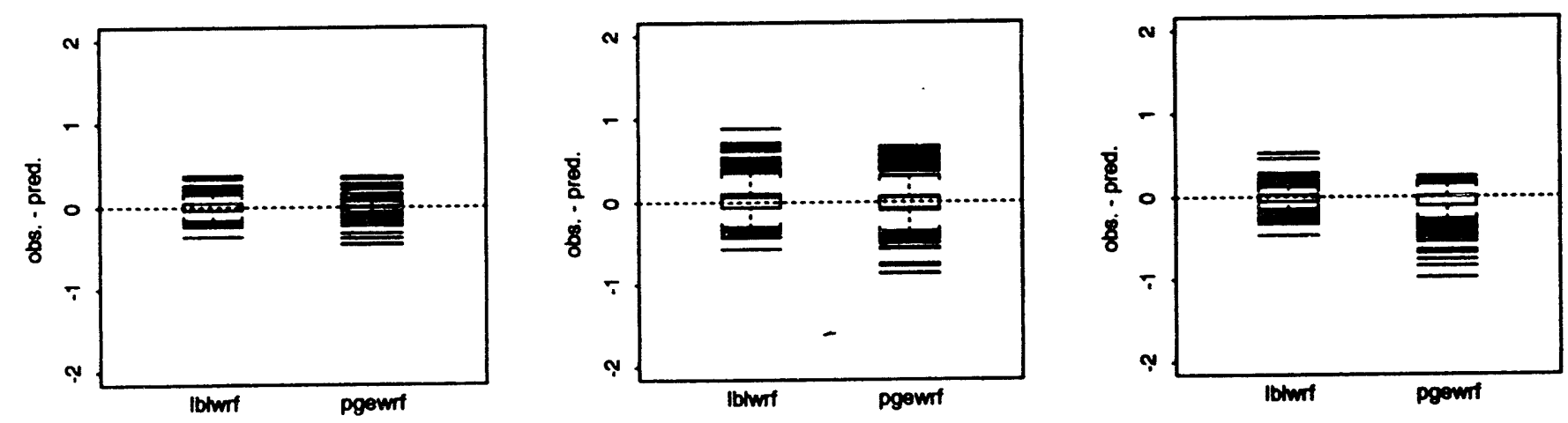

Figure C-4 . Distribution of season-specific model residuals (obs. - pred.) for PG\&E Zone R . 

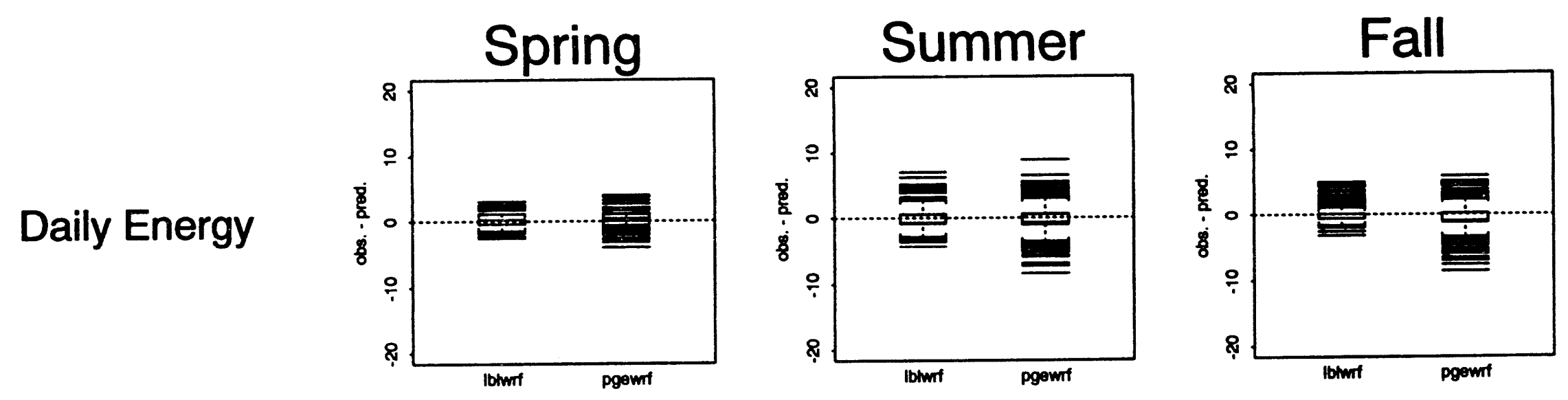

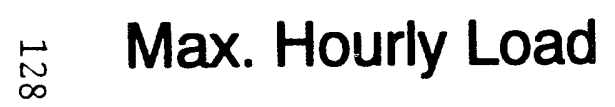
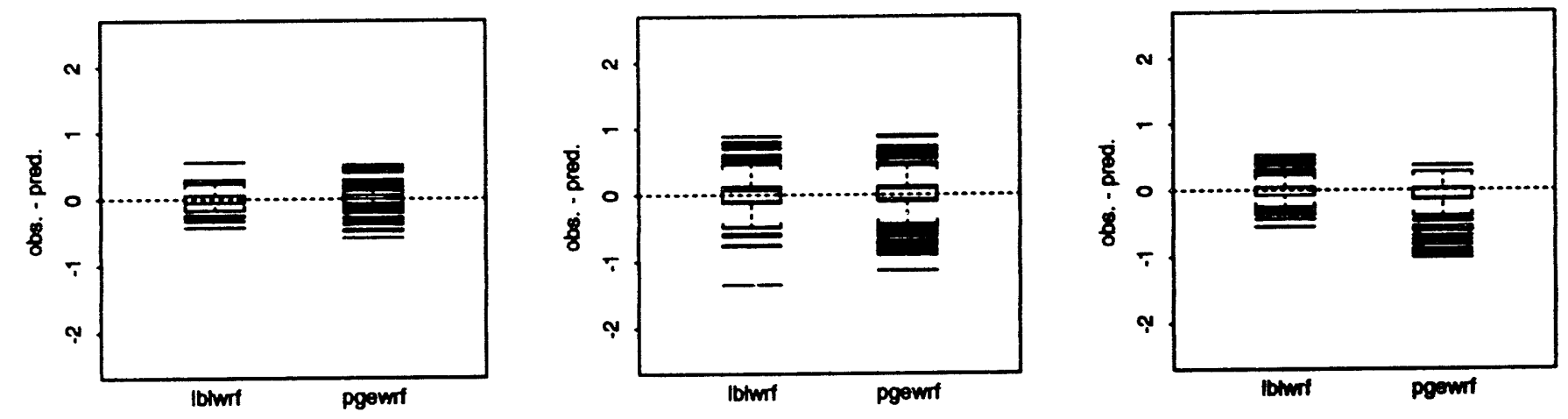

4 p.m. Load
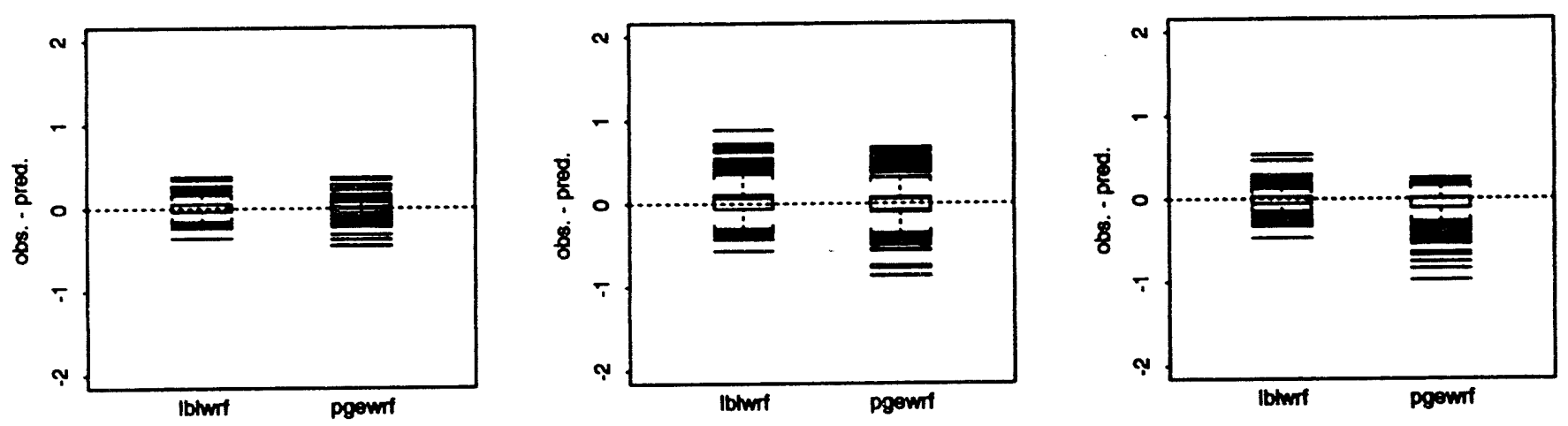

Figure C-5. Distribution of season-specific model residuals (obs. - pred.) for PG\&E Zone S . 


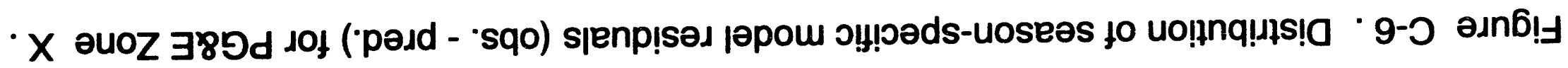
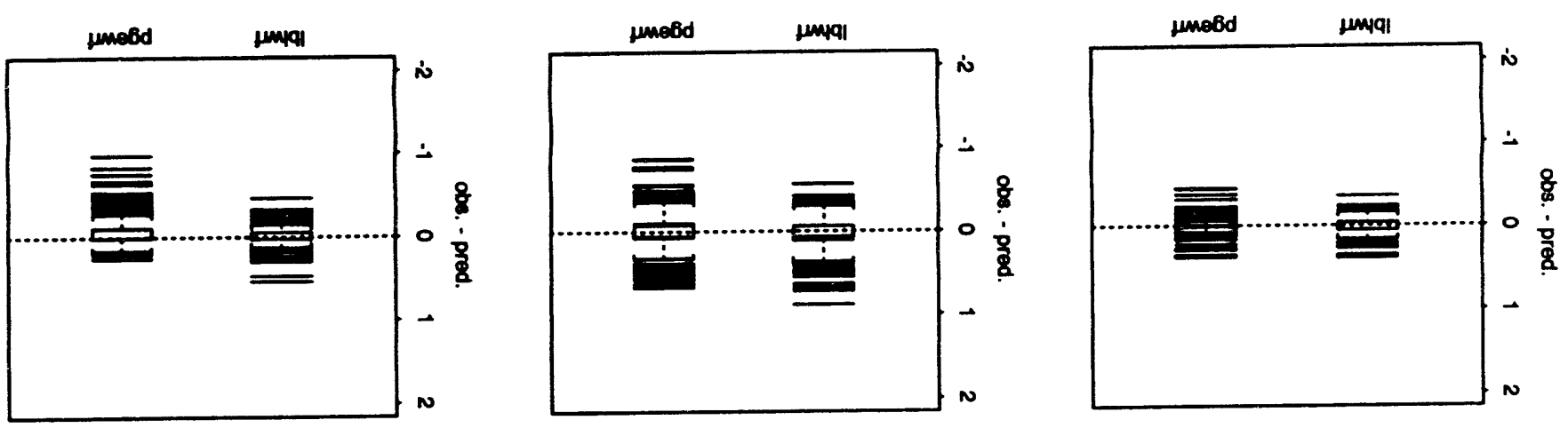

peof $\cdot w \cdot d t$
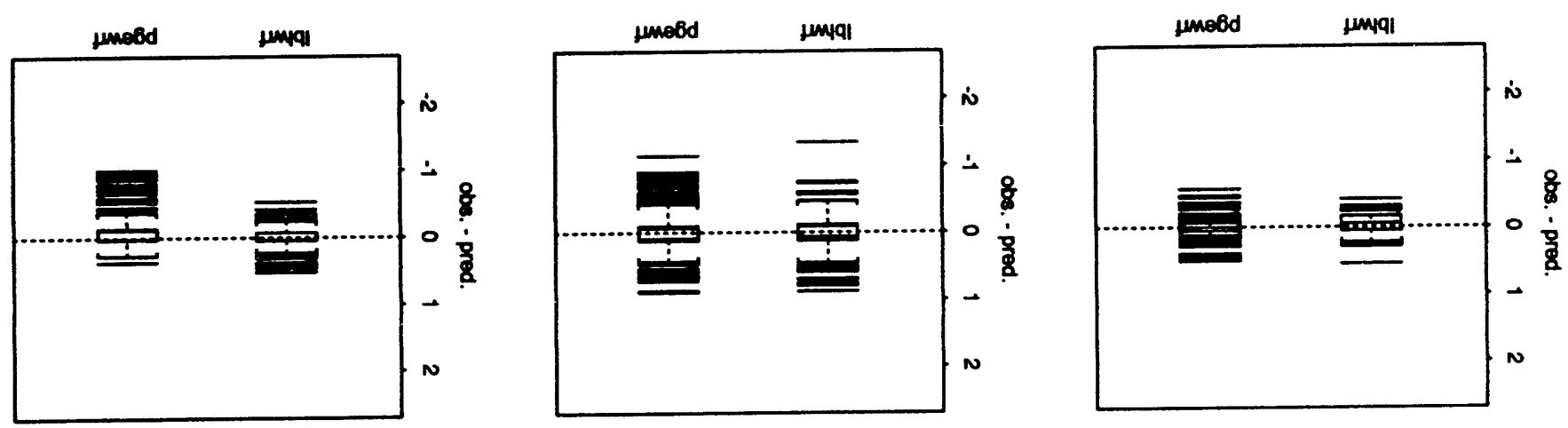

peo7 אןnoH 'xeW

$\stackrel{2}{\sim}$

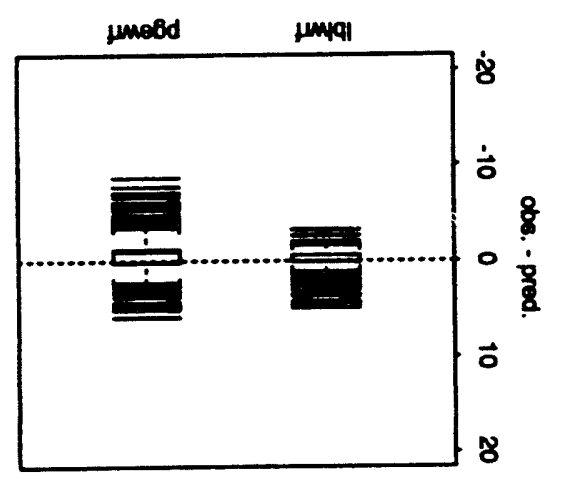

||巳」

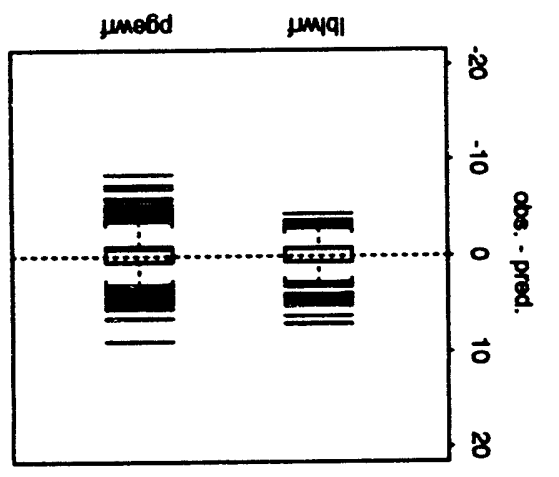

jouunS

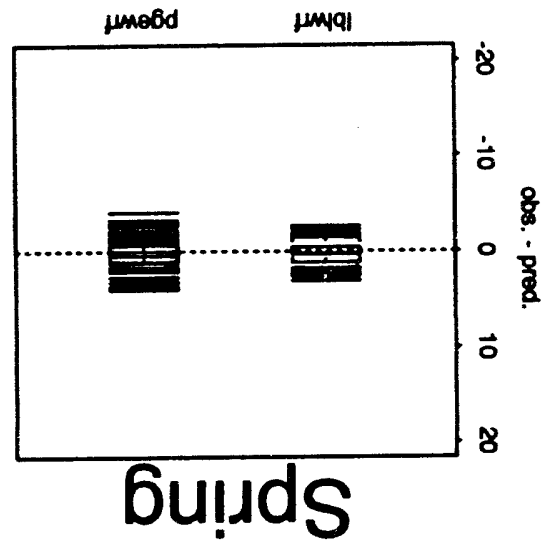

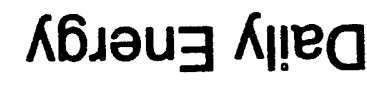




\section{HELM Model Implementation of Project Results}

This appendix documents the HELM input files provided as products of the current project, and summarizes an important issue in using HELM. ${ }^{50}$

We prepared HELM input files for the "final" versions of the models described in Chapters 3 and 4 , which combine daily energy forecasts with bin-specific 24-hour load shapes. We developed six sets of inputs, one for each of the three CEC regions studied (CEC regions 2, 3, and 4), and one for each of the three PG\&E zones studied (PG\&E zones $\mathbf{R}, \mathbf{S}$, and $\mathrm{X}$ ). The input files developed for each of these geographic areas consist of one Daily WRF files and one Load Shape Representation file. The Daily WRF files contain the final daily energy regression models reported in Chapter 3 (Tables 3-3 and 3-4). The Load Shape Representation files contain load shape libraries, which are sets of 24-hour load shapes corresponding to the temperature, season, and day-type bins specified in Table 4-1 (for PG\&E zones) and Table 4-2 (for CEC regions). Model specifications are completed by day type and season definitions (which are defined in this report and in our calendar files), and definitions of the variables used in the models (which are defined in Table 3-2).

We have already discussed two issues relevant to the use of project results within HELM, including the format and number of variables that can be used to specify WRFs, and the ease of using the WRFs, both described in Chapter 3. In this appendix, we discuss the reasonableness of HELM model forecasts developed using project results, in the context of current PG\&E and CEC forecasting procedures. Specifically, we comment on the issue of negative load forecasts resulting from use of LBL's WRF and the weather files currently used by PG\&E and CEC in forecasting.

\footnotetext{
so Because the production version of HELM 2.0 was not available when we began developing and testing input files, we used the beta release of HELM 2.0.
} 


\section{D.1 Background}

The nature of linear regression, which is the basis for the HELM WRFs, means that their use may produce negative values for loads. The occurrence of such loads depends on the forecast weather data used in the HELM. Both CEC and PG\&E have reported obtaining negative loads while using preliminary LBL WRFs along with their respective "typical weather" files, and it is likely that any "reasonable" typical weather data for the areas studied would likewise result in a few (but not many) negative loads.

The occurrence of such negative load forecasts is statistically acceptable in the context of forecasting. However, because negative loads are theoretically impossible, they may be awkward to use: the occurrence of such as a prediction can be conceptually discomforting and could conceivably result in computer-related difficulties in the postprocessing of model output.

\section{D.2 Approach and Findings}

CEC and PG\&E requested that we investigate the occurrence of negative loads in order to develop recommendations on how they might best be dealt with. We obtained the "typical weather" files used by $C E C^{51}$ and PG\&E ${ }^{52}$ in their ER-94 HELM forecasts runs. We generated HELM model predictions using these typical weather files (as both the forecast and the base weather file) and examined the occurrence of negative loads. Note that the hourly loads for a day are zero if and only if the daily load is zero, since application of the binned load shape implies multiplication of the daily energy load shape by a positive scale.

We examined the occurrence of negative loads for each CEC region and PG\&E zone, determining for each the number of negative loads, the total (negative) energy these loads represent, and the percentage this total (of negative loads) is of the forecast annual energy. Negative loads account for $1.3,0.1$, and 1.1 percent of annual energy for CEC regions 2,3 , and 4 respectively; days with negative loads account for $6.3,2.5$, and 4.1 percent of the 365 days for these regions, respectively. Of the three PG\&E zones $R$, $\mathrm{S}$, and $\mathrm{X}$, only the forecast for zone $\mathrm{R}$ included any negative load forecasts. Negative

\footnotetext{
51 These are the files PGE92.WTR in the RES subdirectories of geographic area directories (SACTO, FESN, and SANJOSE for CEC regions 2, 3, and 4 respectively), which were transmitted with a memorandum dated August 31, 1993.

52 These are the files CZRRES.DAT, CZSRES.DAT, and CZXRES.DAT (for PG\&E zones R, S, and X respectively) received in September 1993.
} 
load forecasts for zone $\mathbf{R}$ accounted for 2.4 percent of annual forecast energy, and occurred on 1.4 percent of the 365 days.

\section{D.3 Discussion}

Options for treating negative loads, apart from ignoring them, include: (1) replacing negative loads by zero; (2) modifying the WRFs; and (3) defining new bins.

Option 1, replacing negative loads by zero, will result in an overestimate (relative to the model as originally specified) of total energy for the forecast period. That is, if forecast weather and base weather are the same, the total forecast load (if negatives are interpreted as zeros) will be higher than the energy specified in the forecast. The overestimate would be small (equal to the sum as negative loads as a percent of annual energy), but can probably not be considered negligible: in our case, for CEC regions 2 and 4 and PG\&E zone $R$ the total of negative loads is more than one percent of total annual load, as shown above (1.3 percent, 1.1 percent, and 2.4 percent, respectively. Further, resetting negative loads to zero may be computationally awkward, since it requires post-processing of the HELM output.

Option 2, modifying the WRFs, has been used both by CEC and PG\&E. It is in general not mathematically straightforward to calculate minimal adjustments to eliminate negative loads, since each WRF uses a variety of weather-based variables which are not a function of AVGDRY used to define the bins. Furthermore, adjustments necessary depend also on the particular weather used. Trial and error may be the easiest method of making these adjustments. Such ad hoc adjustments can result in substantial changes in HELM forecasted loads, although they will not necessarily do so. For example, to get rid of negative loads in our CEC region 2 forecasts, we adjusted the TCHANGE coefficients (which were negative values) for the Spring and Summer region 2 WRFs. While we tried to make these adjustments as small as possible, the adjustments resulted in a substantial change in peak load, reduced the predicted peak load by 3.5 percent. In our test case for each region, we noticed that the negative loads occurred only in late Spring and early Summer.

Option 3, defining new bins, is again not a straightforward calculation either. The necessary adjustments may again be most easily achieved by trial and error. Option 3 is perhaps the most acceptable of the options sketched here (excluding "do nothing"), since peak predicted load would not be affected by such an adjustment. There are apparently (at least) two ways to make the adjustment: (1) shifting the lower bound of the lowest load shape bin slightly higher, or (2) possibly using a secondary variable (in addition to AVGDRY) to define an additional set of bins for each season and region. 


\section{D.4 Recommendation}

In view of the small amount of energy affected and the necessarily somewhat ad hoc approaches required to implement the alternative, we recommend that negative loads be retained, as predicted. In the context of the overall system load shape, the effect of these negative loads will be negligible. Accepting negative loads will also preserve the consistency between the annual energy and load shape forecasts. 

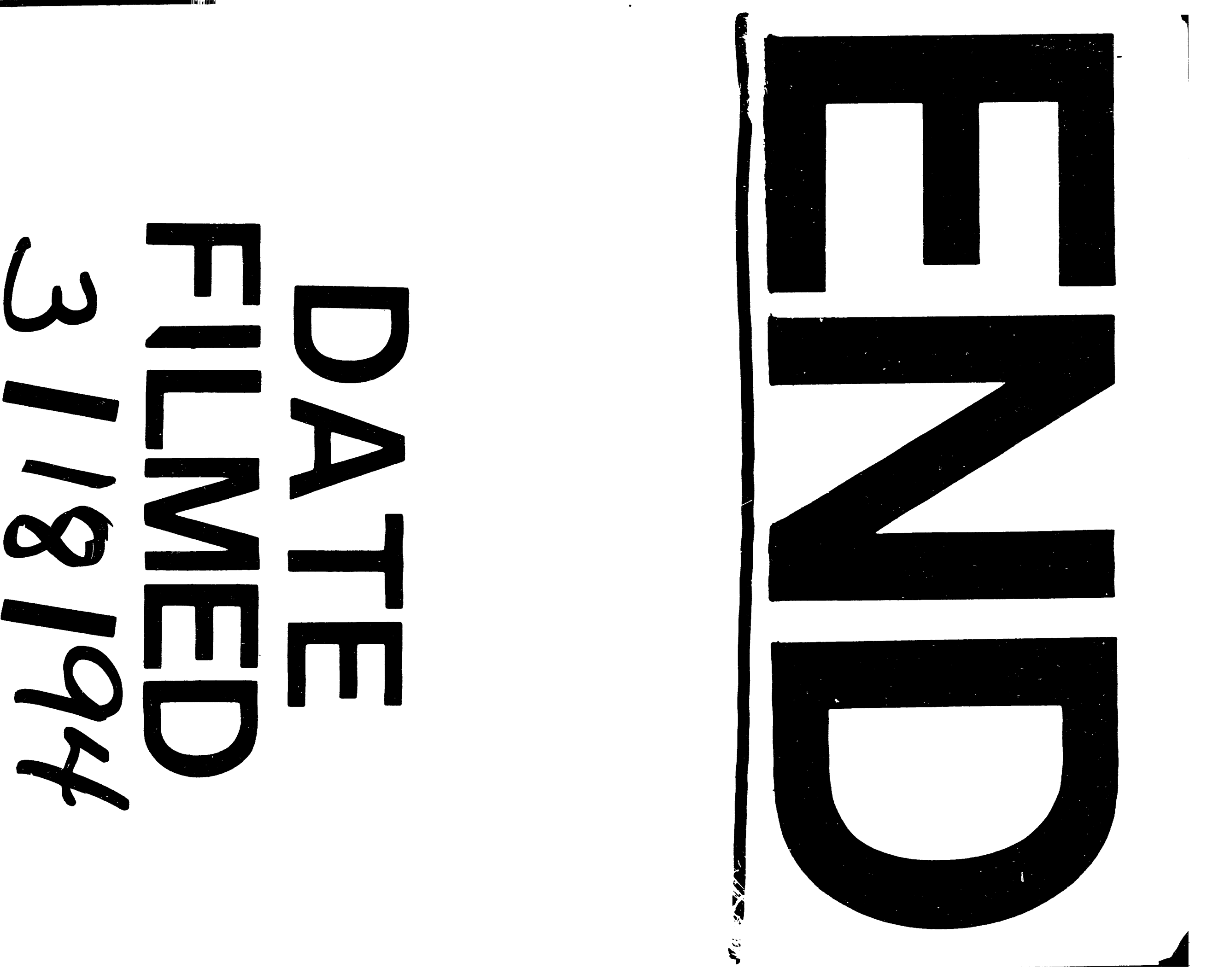
